\title{
STRUCTURE AND DIMENSION OF GLOBAL BRANCHES OF SOLUTIONS TO MULTIPARAMETER NONLINEAR EQUATIONS
}

BY

\author{
J. IZE, I. MASSABÓ, J. PEJSACHOWICZ AND A. VIGNOLI ${ }^{1}$
}

\begin{abstract}
This paper is concerned with the topological dimension of global branches of solutions appearing in different problems of Nonlinear Analysis, in particular multiparameter (including infinite dimensional) continuation and bifurcation problems. By considering an extension of the notion of essential maps defined on sets and using elementary point set topology, we are able to unify and extend, in a selfcontained fashion, most of the recent results on such problems. Our theory applies whenever any generalized degree theory with the boundary dependence property may be used, but with no need of algebraic structures. Our applications to continuation and bifurcation follow from the nontriviality of a local invariant, in the stable homotopy group of a sphere, and give information on the local dimension and behavior of the sets of solutions, of bifurcation points and of continuation points.
\end{abstract}

1. Introduction. This paper arose from an attempt to unify, clarify and extend some recent work on the topological dimension of global branches of solutions appearing in different problems of Nonlinear Analysis.

We shall consider equations of the form

$$
f(x, \lambda)=0, \quad \lambda \in \Lambda,
$$

where $f: E \times \Lambda \rightarrow F$ is a continuous map (see, however, $\S 3$ ), $E, F$ are Banach spaces and $\Lambda$ is the "parameter space" which is a (not necessarily finite dimensional) Banach space.

We will look for global branches of solutions of problem (1.1), their behavior and structure, paying particular attention to their (local) topological dimension.

A first, rather natural, but naïve approach to this type of problem is that of reducing it to a one-dimensional parameter problem by looking at one-dimensional "slices" of the parameter space and by applying to the reduced case the already known results such as the Leray-Schauder continuation principle [LS] or, say, the Rabinowitz global alternative for bifurcation problems [R]. By doing so one obtains a family of one-parameter global branches, each member of which having a known

Received by the editors November 4, 1983.

1980 Mathematics Subject Classification. Primary 58B05, 58E07; Secondary 47H15, 54F45.

Key words and phrases. Global branches of solutions, multiparameter continuation problems, multiparameter bifurcation problems, covering dimension, essential maps, cantor manifolds.

${ }^{1}$ Work performed while the fourth named author was visiting the Department of Mathematics and Mechanics, IIMAS-UNAM, México. 
behavior. The problem that now arises is to understand how these one-dimensional branches "patch together". In absence of differentiable structures (such as manifold structures) a good substitute for a direct approach in describing the global structure of the set of solutions of problem (1.1) is that of the topological covering dimension. A first result in this direction, when the map $f: E \times \Lambda \rightarrow E$ has the form

$$
f(x, \lambda)=x-k(x, \lambda), \quad \lambda \in \mathbf{R}^{n},
$$

where $k: E \times \mathbf{R}^{n} \rightarrow E$ is compact, has been successfully undertaken in [AA,I]. In this last paper a global multidimensional version of the global bifurcation result contained in $[\mathbf{R}]$ was obtained.

For problems of the form (1.2) the well-known Leray-Schauder continuation principle was studied in [MP]. Extensions of the same problem, when $\lambda$ varies in an infinite dimensional Banach space, were obtained in [AA,II and AMP] respectively. Further refinements and simplifications of the Leray-Schauder continuation principle, by allowing the map $f$ to belong to the wider class of $A$-proper maps, has been obtained in [FMP,I].

Finally, [FMP,II] contains a unified approach to the finite multiparameter version of the implicit function theorem, the continuation principle and the global bifurcation results. All of the above mentioned papers use cohomology theories (in particular Čech cohomology).

In this paper we will deal, at the same time, with both the finite and infinite dimensional parameter cases.

Another important point is that we will obtain our results by elementary means, in the sense that our tools will be based essentially on point-set topology. This is, in contrast to the above papers, where the more sophisticated machinery of Algebraic Topology was exploited in some depth.

We will reach our goal by first introducing an appropriate and very broad class of maps and then prove, again in elementary terms, a general theorem that will include as particular cases all of the previously mentioned results.

We wish to give here an idea of what our class of maps looks like and how wide it is, referring the reader to $\$ 2$ for further details and extensions.

Let $E, G$ be Banach spaces, $U \subset E$ open and $S$ an arbitrary subset of $E$. A continuous map $g: U \rightarrow G$ defined on the (not necessarily bounded) open set $U$ is called zero-epi (0-epi) on $S \cap U$ provided that $\overline{g^{-1}(0) \cap S}$ is a bounded subset of $U$ and the equation $g(x)=h(x)$ is solvable in $S \cap U$ for any compact map $h: E \rightarrow G$ whose support is bounded and contained in $U$.

In the case when $S$ coincides with the whole space $E$ (recall that $S \subset E$ is arbitrary), the above class coincides with that introduced in [FMV,II], where it has been shown, in particular, that when the map $g$ is a compact perturbation of the identity, then $g$ is 0 -epi provided that the Leray-Schauder topological degree $\operatorname{deg}_{\mathrm{LS}}(g, U, 0)$ is defined and different from zero. Also, monotone maps are 0-epi under suitable assumptions (see [FMV,II]).

We shall indicate here that in fact our class of maps contains many more types of maps than those given above. In particular, it contains any class of maps for which a classical degree theory, satisfying the boundary dependence property under compact 
perturbations, is defined (e.g. $\boldsymbol{\kappa}$-set-contractive and condensing vector fields [ $\mathbf{N}$ and $\mathbf{S}]$; maps of type $S_{+}[\mathbf{B r}]$; $A$-proper maps [BP]; coincidence degree [M]; etc.). Indeed, it suffices that the degree theory under consideration satisfies

$$
\operatorname{deg}(g-h, U, 0)=\operatorname{deg}(g, U, 0)
$$

for any compact map $h$ having bounded support contained in $U$.

The class of 0 -epi maps also includes that of compact vector fields which are essential in the sense of A. Granas [G]. This last case furnishes an example of a class of maps for which the topological degree automatically vanishes (the image of the compact vector field is contained in a proper subspace) and, nevertheless, is 0-epi. We shall return to this point in $\S 2$, giving an example of a map from the real line into itself, having zero topological degree and being 0 -epi. For such a class of compact vector fields a generalized degree theory (stable cohomotopy) has been constructed in [GG].

A further important class of 0-epi maps is represented by that of (not necessarily smooth) compact perturbations of nonlinear $C^{1}$ Fredholm maps of nonnegative index. For this class of maps a generalized degree (bordisms and equivalent theories) has been constructed in [BZS]. If a certain map has a nonvanishing generalized degree in the sense of [BZS], then this map is 0-epi.

In the case when $S$ is given by the zeros (i.e., $S=f^{-1}(0)$ ) of a continuous function $f: U \rightarrow F$, where $F$ is another Banach space, then our concept of 0 -epi map on $S \cap U$ gives an extension of the class of zero-regularizable maps, introduced in [FP], in order to study the existence of unbounded components of solutions of nonlinear equations. In [FP] the pair $(f, g)$ is required to be 0 -epi on $U$. This clearly implies that $g$ is 0 -epi on $S \cap U$ (see Property 2.9 in \$2). A sufficient condition for the pair $(f, g)$ to be 0 -epi on $U$ is that the Leray-Schauder degree of the pair, when defined, is nonzero. This idea, together with Čech cohomology, was used in [FMP,II] to obtain their dimension results.

Note however that $g$ may be 0 -epi on $f^{-1}(0) \cap U$ and $(f, g)$ not 0 -epi on $U$. In fact, if $E=\mathbf{R}^{2}, F=G=\mathbf{R}, f(x, y)=x^{2}-1$ and $g(x, y)=y$, then the index of $(f, g)$ at $( \pm 1,0)$ is \pm 1 , so that the degree of $(f, g)$ with respect to any open set $U$ containing $( \pm 1,0)$, is zero. From Remark 2.7 it follows that $(f, g)$ is not 0 -epi on $U$ if $U$ is connected. On the other hand, if $y-h(x, y) \neq 0$ on $S \cap U$ for some $h$ with bounded support contained in $U$, then $y-h(1, y) \neq 0$ which is not possible since this map has degree 1 on the intersection of $U$ with the line $x=1$. Hence, $g$ is 0 -epi on $S \cap U$, where $S=f^{-1}(0)$. In this example the fact that $S$ is not connected does not play any role (replace $x^{2}-1$ by $\left(x^{2}-1\right)(y+1)$ or by $\left.x^{2}\right)$.

Note that if zero is a regular value of $f$, then

$$
\operatorname{deg}((f, g) ; U, 0)=\operatorname{deg}\left(g, f^{-1}(0) \cap U, 0\right)
$$

(see [FMP,I]). This is false in general even if $S$ is a manifold (the above example with $f(x, y)=x^{2}$ shows it).

In our applications we will be interested in a subset $S$ of $f^{-1}(0)$ so that one has to know the properties of $g$ only on $S$ (this usually leads to a relaxation of the compactness hypotheses on $f$ ). This consideration prompted us to deal directly with an arbitrary set $S$, obtaining shorter proofs and more refined results. 
We would like to remark that we are also dealing with the notion of essentiality (if $U$ is bounded, then $g$ is 0 -epi on $S \cap \bar{U}$ means that $g: S \cap \partial U \rightarrow G \backslash\{0\}$ has no nonvanishing extension to $S \cap \bar{U}$ ). Thus it is clear that, if some additional hypotheses are put on $S$, then one may construct, in the spirit of [GG], a generalized stable cohomotopy theory relative to $S$ and hence a generalized degree theory for maps defined on $S$ (similar constructions could be carried over for the other topological invariants mentioned above). In this way we would obtain algebraic properties for these invariants. In this paper we will use only their nontriviality.

In order to give an idea of the type of results that we are going to obtain let us subsume the main result of this paper.

Let $g: U \rightarrow G$ be 0 -epi on $S \cap U$, sending bounded sets into bounded sets; then the following alternative holds: Either $S \cap U$ is unbounded, or $\bar{S} \cap \partial U \neq \varnothing$. If, moreover, $g$ is proper on bounded and closed subsets of $S \cap U$, then there exists a minimal connected subset $\Sigma$ of $S \cap U$ such that $g^{-1}(0) \cap \Sigma \neq \varnothing$ and has covering dimension at each point at least $\operatorname{dim} G$. Furthermore, $\Sigma$ is either unbounded, or $\bar{\Sigma} \cap \partial U \neq \varnothing$. Finally, $\Sigma$ is minimal for any map homotopic to $g$.

Using this result (or the more complete version represented by Theorem 3.1 in §3) we will obtain, in a unified fashion, the multidimensional versions of the LeraySchauder continuation principle, the global bifurcation alternative of Rabinowitz and a global version of the classical implicit function theorem.

Our main result will be exploited to study global multiparameter problems that cannot be tackled with the techniques used in [AA,I; AA,II; FMP,I and FMP,II] (see $\S 4)$.

This paper consists of this Introduction and three further sections. In $\S 2$ we give the definition and prove the main properties of zero-epi (zero-essential) maps on $S \cap U$. In $\S 3$ we obtain the main result of this paper and in $\S 4$ we give applications of our result to the global implicit function theorem, continuation principle and global bifurcation.

As regards the length of this paper, we would like to observe that we start from scratch, building up a complete theory for zero-epi maps on $S \cap U$ and the paper itself, as a whole, is intended to be as selfcontained as possible.

The reader may notice that our list of references is rather short. This is due to the fact that we have restricted our attention mainly to those previous results on the local topological dimension of global branches of solutions of nonlinear equations. We do not mention, unless strictly necessary, the wealth of global results that have been obtained by several authors, starting from the pioneering work of Leray and Schauder [LS]. The interest in this area had been renewed, something more than a decade ago, by the work of Rabinowitz [R].

Finally, we wish to mention that in a forthcoming paper we exploit similar (suitably modified) ideas to obtain results on the topological dimension of global branches for equivariant problems (e.g. global Hopf bifurcation phenomenae).

2. Zero-epi and zero-essential maps. In what follows $E, G$ are Banach spaces, $U$ is an open, not necessarily bounded, subset of $E$, and $S$, unless otherwise specified, will stand for an arbitrary subset of $E$. 
In this section we introduce and study two new classes of maps. Namely we introduce the concept of zero-epi and zero-essential maps on $S \cap U$ (and on $S \cap \bar{U})$. These two classes turn out to be equivalent. We show this immediately after giving the corresponding definitions. We state next the most elementary properties of these maps and then prove the homotopy invariance which is one of the most important properties of 0-epi and 0-essential maps on $S \cap U(S \cap \bar{U})$.

Next, we state and prove several relevant consequences of the homotopy principle which will be of importance in the remainder of the paper.

Finally, we close this section by introducing the broader class of sectionally zero-epi (zero-essential) maps that will play a fundamental role in proving results for the infinite dimensional parameter case.

\section{A. Definitions.}

Definition 2.1. Let $E, G, U$ and $S$ be as above and let $g: U \rightarrow G$ be a continuous map. We say that $g$ is admissible on $S \cap U$ if there exists an open and bounded subset $V_{0}$ such that $g^{-1}(0) \cap S \subset V_{0} \subset \bar{V}_{0} \subset U$.

In the case when $g: \bar{U} \rightarrow G$ is defined on the closure $\bar{U}$ of $U$, we say that $g$ is admissible on $S \cap \bar{U}$ if there exists an open and bounded subset $V_{0}$ such that $g^{-1}(0) \cap S \subset V_{0} \subset U$. This is, of course, equivalent to saying that $g^{-1}(0) \cap S$ is a bounded subset of $U$.

Note that if $g^{-1}(0) \cap S$ is bounded, closed and contained in $U$, then the existence of $V_{0}$ with the above properties follows from the normality of $E$.

Definition 2.2. Let $g$ be admissible on $S \cap U(S \cap \bar{U})$. The map $g$ is called zero-epi on $S \cap U(S \cap \bar{U})$ if the equation $g(x)=h(x)$ has a solution in $S \cap U$ for any compact map $h: E \rightarrow G$ with supp $h$ bounded and contained in $U(\{x \in E$ : $h(x) \neq 0\} \subset U$ or, equivalently, supp $h \subset \bar{U}$ and $h(x)=0$ for all $x \in \partial U)$, where supp $h=\operatorname{closure}\{x \in E: h(x) \neq 0\}$ is the support of the map $h$.

Note that in the case when the map $g$ is defined on $\bar{U}$ the solutions of $g(x)=h(x)$ are in $S \cap U$ since $h(x)=0$ on $\partial U$ and $g$ is admissible on $S \cap \bar{U}$. The class of maps introduced in Definition 2.2 is a generalization and a refinement of the concept of zero-epi map introduced in [FMV,II].

Definition 2.3. Let $g$ be admissible on $S \cap U(S \cap \bar{U})$. The map $g$ is called zero-essential on $S \cap U(S \cap \bar{U})$ if for any open and bounded set $V$ such that $g^{-1}(0) \cap S \subset V \subset \bar{V} \subset U\left(g^{-1}(0) \cap S \subset V \subset U\right)$, any continuous extension $\tilde{g}$ : $\bar{V} \rightarrow G$ of $g: \partial V \rightarrow G$, with $g-\tilde{g}$ compact on $\bar{V}$, has a zero on $S \cap V$.

We would like to point out that the set $S$ above may coincide with the whole Banach space $E$.

Proposition 2.1. If $U$ is bounded and $g: \bar{U} \rightarrow G$ is nonvanishing on $S \cap \partial U$, then $g$ is 0-essential on $S \cap \bar{U}$ if and only if any extension $\tilde{g}: \bar{U} \rightarrow G$ of $\left.g\right|_{\partial U}$ with $g-\tilde{g}$ compact on $\bar{U}$ has a zero on $S \cap U$.

Proof (Only if ). Take $V=U$.

(If ). Assume that there exists an open set $V$ such that $g^{-1}(0) \cap S \subset V \subset U$ and $\tilde{g}: \bar{V} \rightarrow G$ extends $\left.g\right|_{\partial V}$ with $g-\tilde{g}$ compact and $\tilde{g} \neq 0$ on $S \cap V$. Then, the map $g_{1}$ defined as $\tilde{g}$ on $\bar{V}$ and $g$ on $\bar{U} \backslash V$ is nonvanishing on $S \cap \bar{U}$. Moreover, $g-g_{1}$ is compact on $\bar{U}$ and $g_{1}$ is an extension of $\left.g\right|_{\partial U}$. Q.E.D. 
Note that the rather involved definition of 0 -essential map in the case of unbounded $U$ is due to the fact that, unlike the excision property for the topological degree, a map $g$ may be 0 -essential on $S \cap U$ and not 0 -essential on $S \cap W$, where $W$ is an open set containing $U$. The following simple example shows this. Let $E=S=\mathbf{R}$ and let $g: \mathbf{R} \rightarrow \mathbf{R}$ be defined by $g(x)=x^{2}-1$. The map $g$ is 0 -essential on $S \cap U$ (and on $S \cap \bar{U}$ ), with $U=\mathbf{R} \backslash\{0\}$, but it is not 0 -essential on $S \cap W$, where $W=\mathbf{R}$. Indeed, on one hand $g$ changes sign on the boundary $\partial V$ of any open and bounded subset $V \subset U$ such that $g^{-1}(0) \subset V$ and hence is 0 -essential on $S \cap U$. On the other hand, by taking the open subset $V$ of $W$ defined by the open interval $V=(-a, a), a>1$, we have that the constant function $\tilde{g}=a^{2}-1$ is a nonvanishing extension of $\left.g\right|_{\partial V}$.

Warning about the notation. The above example shows that for our definition to be 0 -epi on $S \cap(\overline{\mathbf{R} \backslash\{0\}})$ is not the same as to be 0 -epi on $S \cap \mathbf{R}$.

A justification of the term 0 -essential on $S \cap U$ will be given after Remark 2.4 below.

Proposition 2.2. The map $g$ is 0-epi on $S \cap U(S \cap \bar{U})$ if and only if $g$ is 0 -essential on $S \cap U(S \cap \bar{U})$.

Proof. (If ). Let $h$ be as in Definition 2.2 and set $V=\{x \in E: h(x) \neq 0\} \cup V_{0}$, where $V_{0}$ is as in Definition 2.1. Clearly, $V$ is open, bounded and $\bar{V}=\operatorname{supp} h \cup \bar{V}_{0}$. Moreover, $V$ satisfies the properties of Definition 2.3. Since $h$ vanishes on $\partial V$, then $\left.\tilde{g} \equiv(g-h)\right|_{\bar{V}}$ is an extension of $\left.g\right|_{\partial V}$ satisfying the requirements of Definition 2.3 and, as such, $\tilde{g}$ has a zero on $S \cap V$.

(Only if ). Let $V$ and $\tilde{g}$ be as in Definition 2.3. Define

$$
h= \begin{cases}g-\tilde{g} & \text { on } \bar{V}, \\ 0 & \text { on } E \backslash V .\end{cases}
$$

Then supp $h \subset \bar{V}$ and $h$ satisfies the requirements of Definition 2.2. Hence, the equation $g(x)=h(x)$ must have a solution $x \in S \cap U$. Since $x$ cannot be in $E \backslash \bar{V}$, then it has to be in $S \cap V$. Q.E.D.

REMARK 2.1. In the first implication of Proposition 2.2 the set $g^{-1}(0) \cap S$ does not play any role, while in the second the only thing needed is $g^{-1}(0) \cap S \subset V$ so that one may replace $g^{-1}(0) \cap S$ by any set $K$ with the property $g^{-1}(0) \cap S \subset K \subset V$.

REMARK 2.2. The following is a variant of Definitions 2.2 and 2.3.

Instead of requiring $h$ compact on $E(g-\tilde{g}$ compact on $\bar{V})$ one may require

$h$ compact on $S$ ( $g-\tilde{g}$ compact on $S \cap \bar{V}$ ) or, equivalently, $h$ compact on $S \cap U(S \cap \bar{U})$, since $h$ is identically zero outside $U$.

Notice that this is a stronger requirement on $g$ and that Proposition 2.2 is still valid in this context. Furthermore, if $S \cap U$ is closed in $U$ ( $S \cap \bar{U}$ is closed), then the above definitions are equivalent to Definitions 2.2 and 2.3. In fact, the map $h$ 
restricted to the set $A \equiv(S \cap \operatorname{supp} h) \cup\{x \in E: h(x)=0\}$ is compact, since $h$ is compact on $S \cap U$. Moreover, the inclusion supp $h \subset U$ (supp $h \subset \bar{U}$ ) implies that $S \cap \operatorname{supp} h$ is closed and, thus, so is $A$. Hence, by Dugundji's extension theorem [D, p. 188], the restriction $\left.h\right|_{A}$ has a compact extension $\tilde{h}$ to the whole space $E$. Clearly, $\{x \in E: \tilde{h}(x) \neq 0\} \subset\{x \in E: h(x) \neq 0\}$ and from $(*)$ above the equation $g(x)=$ $\tilde{h}(x)$ has a solution in $S \cap U$, where $\tilde{h}=h$. An analogous proof holds for Definition 2.3.

REMARK 2.3. An even stronger assumption on $g$ would be to require $h(g-\tilde{g})$ to be only continuous. It is easily seen that Proposition 2.2 is still valid in this context. However, if $G$ is infinite dimensional this class of maps may be very small. For example, the identity map (with $S=E$ and $U=$ unit ball of $E$ ) is not 0 -epi on $S \cap U$. Indeed, the identity restricted to $\partial U$ has a nonzero continuous extension given by any continuous retraction of the unit closed ball onto its boundary (such a retraction exists since $\operatorname{dim} G=+\infty$ ).

Other variants could be obtained by asking different properties on the map $h$ such as $k$-set-contractivity, $A$-properness, equivariance, etc.

REMARK 2.4. A relative (to $S$ ) variant of Definition 2.3 could be given by requiring that any extension of $g: S \cap \partial V \rightarrow G \backslash\{0\}$ to $S \cap \bar{V}$ such that $g-\tilde{g}$ is compact on $S \cap \bar{V}$ has a zero in $S \cap V$.

Note that the above variant is weaker than Definition 2.3. However, if $S \cap U$ is closed in $U$ ( $S \cap \bar{U}$ is closed), then both definitions coincide: one has to extend $\tilde{g}$, defined only on $S \cap \bar{V}$, to a map $\hat{g}$ which coincides with $g$ on $\partial V$. This is possible considering the compact map defined on the closed set $(S \cap \bar{V}) \cup \partial V$ as $g-\tilde{g}$ on $S \cap \bar{V}$ and zero on $\partial V$. Now, use Dugundji's extension theorem and proceed as in Remark 2.2.

The above conclusion holds also in the context where $h$ is assumed to be only continuous, since one has also continuous extensions [D, p. 188].

Before going into the exposition of the elementary properties of 0 -epi maps we would like to, at least partially, justify our terminology of 0-essential map on $S \cap \bar{U}$. Taking into account Proposition 2.1 and the above, if $U$ is bounded and $S \cap \bar{U}$ is closed, then the map $g$ is 0 -essential on $S \cap \bar{U}$ if and only if the restriction $\left.g\right|_{S \cap \partial U}$ is essential (in the classical sense, see [G]) with respect to $S \cap \bar{U}$.

B. Elementary properties. We list now the first, and more elementary, properties of 0 -epi (and, therefore, of 0-essential) maps on $S \cap U(S \cap \bar{U})$. In what follows $V_{0}$ stands for an open and bounded subset.

Property 2.1 (Existence). If $g$ is 0 -epi on $S \cap U(S \cap \bar{U})$, then $g^{-1}(0) \cap S \neq \varnothing$. This follows at once by taking as $h$ the identically zero map on $E$.

PROPERTY 2.2 (LOCAlization With RESPECT TO THE OPEN SET). If $g$ is 0-epi on $S \cap U(S \cap \bar{U})$, then $g$ is 0-epi on $S \cap V(S \cap \bar{V})$ for any open set $V$ such that $g^{-1}(0) \cap S \subset V_{0} \subset \bar{V}_{0} \subset V \subset U\left(g^{-1}(0) \cap S \subset V \subset U\right)$.

Property 2.3 (Localization With ReSPECt to THE BOUNDARY). If $g$ is 0-epi on $S \cap \bar{U}$ and $g^{-1}(0) \cap S \subset V_{0} \subset \bar{V}_{0} \subset U$, then $g$ is 0-epi on $S \cap U$.

PROPERTY 2.4. If $g$ is 0-epi on $S \cap U$, then $g$ is 0-epi on $S \cap \bar{V}$ for any open set $V$ such that $g^{-1}(0) \cap S \subset V \subset \bar{V} \subset U$. 
Property 2.5. If $g$ is 0 -epi on $S_{1} \cap U\left(S_{1} \cap \bar{U}\right)$ and $S_{2}$ is a set such that $S_{1} \subset S_{2}$ and $g^{-1}(0) \cap S_{2} \subset V_{0} \subset \bar{V}_{0} \subset U\left(g^{-1}(0) \cap S_{2}\right.$ is bounded and contained in $\left.U\right)$, then $g$ is 0-epi on $S_{2} \cap U\left(S_{2} \cap \bar{U}\right)$.

Property 2.6. If $g$ is 0-epi on $S \cap U(S \cap \bar{U})$, then $g$ is 0 -epi on $\left(S \backslash U^{c}\right) \cap U$ $\left(\left(S \backslash U^{c}\right) \cap \bar{U}\right)$.

Proof. This follows from the fact that $g(x)-h(x)=0$ has its solutions on $S \cap U=\left(S \backslash U^{c}\right) \cap U$. Q.E.D.

Property 2.7 (Normalization). If $i: U \hookrightarrow E$ is the inclusion map, $0 \notin \partial U$ and $S$ is any subset of $E$, then the map $i$ is 0 -epi on $S \cap U$ if and only if $0 \in S \cap U$ and the connected component of 0 in $U$ is contained in $S$. In particular, if $U$ is connected, then $S \cap U=U$.

Proof. (Only if). That $0 \in S \cap U$ follows from Property 2.1. If the second assertion is false, then there exists a point $x_{0}$ in the connected component of $U$ containing zero such that $x_{0} \notin S$ (and, obviously, $x_{0} \neq 0$ ). Now, $U$ is an open subset of a Banach space and so is locally path connected. Therefore the component of 0 in $U$ is path connected and open. Let $\sigma(t), t \in[0,1]$, be a path (in $U$ ) from 0 to $x_{0}$. Clearly, $\{\sigma(t)\}$ is compact so that there exists an $\varepsilon$-neighborhood of the path which is contained in the path component. Let $\phi$ be an Urysohn function taking value 1 on the path $\sigma$ and vanishing outside the $\varepsilon$-neighborhood. Put $h(x)=\sigma(\phi(x))$. Since $\sigma(0)=0$, it follows that $\operatorname{supp} h$ is contained in the $\varepsilon$-neighborhood and $\operatorname{supp} h$ is bounded. Taking into account that the map $g(x)=x$ is 0 -epi on $S \cap U$ we obtain that the equation $x-\sigma(\phi(x))=0$ has a solution $\bar{x} \in S \cap U$. The equality $\bar{x}=$ $\sigma(\phi(\bar{x}))$ implies that $\bar{x}$ belongs to the path $\sigma$ and thus $\phi(\bar{x})=1$. Hence $\bar{x}=x_{0} \notin S$.

(If). Assume that $0 \in U$ and that the component $V$ of 0 in $U$ is contained in $S$. Then the fact that the inclusion map is 0-epi on $S \cap U$ follows from a degree argument. In fact, under the above assumptions, the Leray-Schauder topological degree $\operatorname{deg}_{\mathrm{LS}}(I-h, V, 0)=\operatorname{deg}_{\mathrm{LS}}(I, V, 0)=1($ notice that $h(x)=0$ for all $x \in \partial V)$. Thus, $x-h(x)=0$ is solvable in $V$. Q.E.D.

REMARK 2.5. If $g: U \rightarrow G$ is one-to-one and 0-epi on $S \cap U$, then the connected component of $g^{-1}(0)$ in $U$ is contained in $S$. The proof is analogous to that of Property 2.7. It suffices to consider the map $g(\sigma(\phi(x)))$, where $\sigma$ is a path from $g^{-1}(0)$ to $x_{0}$.

REMARK 2.6. The above properties (together with the homotopy property) are very close to those enjoyed by any degree theory except the excision property. Namely, if $g: \bar{U} \rightarrow \mathbf{R}^{n}$ is a continuous map defined on the closure $\bar{U}$ of an open and bounded set $U$ of $\mathbf{R}^{n}$ such that $g^{-1}(0) \subset V \subset U$, where $V$ is open, then the Brouwer topological degree satisfies $\operatorname{deg}(g, V, 0)=\operatorname{deg}(g, U, 0)$. In particular, if this degree is nonzero, then $g$ is 0 -epi on $V$ and also on $U \supset V$ (see also Remark 2.7 below). This is not true in general for 0-epi maps, even in the simple case when $S=E$. For example let $V \subset \mathbf{R}^{n+m}$ be the open (connected) set defined by $V=\left\{x \in \mathbf{R}^{n}, y \in \mathbf{R}^{m}\right.$ : $\left.(\|y\|-a)^{2}+\|x\|^{2}<r^{2}\right\}, a>r$, i.e., $V$ is a full torus. Now, fixing $x \in \mathbf{R}^{n}$ the set $V$ yields the thickened sphere $a-\sqrt{r^{2}-\|x\|^{2}}<\|y\|<a+\sqrt{r^{2}-\|x\|^{2}}$ and, fixing $y \in \mathbf{R}^{m}$ we get the ball $\|x\|<\sqrt{r^{2}-(\|y\|-a)^{2}}$. Therefore $V$ has the homotopy type of $B^{n} \times S^{m-1}$. Consider now the map $g: \mathbf{R}^{n+m} \rightarrow \mathbf{R}^{n+1}$ defined by $g(x, y)=(x$, $\|y\|-a)$ and let $B$ be the following ball $B=\left\{(x, y) \in \mathbf{R}^{n+m}:\|x\|^{2}+\|y\|^{2}<R^{2}\right\}$, 
where $R>a+r$. Clearly, $B \supset V$ and $g(x) \neq 0$ for all $x \in B \backslash V$. Moreover, $g(x, y)=\left(x, \sqrt{R^{2}-\|x\|^{2}}-a\right)$, for all $(x, y) \in \partial B$, which is deformable to $(0, R-$ a) via $\left(t x, \sqrt{R^{2}-t\|x\|^{2}}-a\right)$ and, as such, the restriction $\left.g\right|_{\partial B}$ is inessential and has a nonzero extension to $B$ (see [I, II, p. 154]). Assume now that $\left.g\right|_{\partial V}$ has a nonzero extension $\tilde{g}$ to $\bar{V}$. Write $y$ as $(y, \tilde{y})$ with $\tilde{y} \in \mathbf{R}^{m-1}$; then $g(x, y, 0) \neq 0$ on $V \cap\{\tilde{y}=$ $0\}$, which is the disjoint union of two closed balls in $\mathbf{R}^{n-1}:\left\{(x, y):|x|^{2}+\right.$ $\left.( \pm y-a)^{2}<r^{2}\right\}$. On their boundary $g(x, y)=(x, \pm y-a)$ is a linear map with topological degree \pm 1 , and as such is essential, contradicting the assumption. Hence $g$ is 0-epi on $\bar{V}$.

REMARK 2.7. If $g: \bar{U} \rightarrow \mathbf{R}^{n}$ with $U$ open, bounded and connected in $\mathbf{R}^{n}$, then $g$ is 0 -epi on $U$ if and only if $\operatorname{deg}(g, U, 0) \neq 0$ (see [I, II, p. 161]). In the above example for $n=1$, the set $V$ has two components, the global degree is zero. Nevertheless, $g$ is 0 -epi on $\bar{V}$.

The following result will play a key role in $\S 3$.

Proposition 2.3. Let $g: \bar{U} \rightarrow G$ be continuous and $S$ be any subset of $E$ such that

(i) $g^{-1}(0) \cap S$ is bounded,

(ii) for any closed and bounded subset $D$ of $\partial U$, $\operatorname{dist}(g(\bar{S} \cap D), 0)$ is positive whenever $\bar{S} \cap D \neq \varnothing$.

Then $g$ is 0-epi on $S \cap \bar{U}$ if and only if $g$ is 0 -epi on $S \cap U$.

Proof. (Only if ). Clearly, $g^{-1}(0) \cap \bar{S} \cap \partial U=\varnothing$, so that from the normality of $E$, there is an open and bounded set $V_{0}$ such that $g^{-1}(0) \cap \bar{S} \subset V_{0} \subset \bar{V}_{0} \subset U$ and hence, by Property 2.3, the map $g$ is 0 -epi on $S \cap U$.

(If). Let $h: E \rightarrow G$ be a compact map with supp $h$ bounded, contained in $\bar{U}$ and $h(x)=0$ for all $x \in U^{c}$.

1 st case. If $\bar{S} \cap \operatorname{supp} h \cap \partial U=\varnothing$, then there is a bounded and open set $V$ such that $\bar{S} \cap \operatorname{supp} h \subset V \subset \bar{V} \subset U$. Let $\phi$ be an Urysohn function with $\phi(\bar{S} \cap \operatorname{supp} h)=$ 1 and $\phi\left(V^{c}\right)=0$. Put $\tilde{h}(x)=\phi(x) h(x)$; then $\operatorname{supp} \tilde{h} \subset \operatorname{supp} h \cap \bar{V} \subset U$ so that $g(x)=\tilde{h}(x)$ has a solution $\bar{x} \in S \cap U$. Now, either $h(\bar{x})=0$, in which case $\tilde{h}(\bar{x})=0$, or $\bar{x} \in \operatorname{supp} h$ and thus $\tilde{h}(\bar{x})=h(\bar{x})$.

2 nd case. If $\bar{S} \cap \operatorname{supp} h \cap \partial U \neq \varnothing$, then $\|g(\bar{S} \cap \operatorname{supp} h \cap \partial U)\| \geqslant d>0$. The first step will be to replace $h$ with another compact map having bounded support contained in $\bar{U}$ and $h(x)=0$ on $\partial U$, also called $h$, for which the inequality $\|g(\operatorname{supp} h \cap \partial U)\| \geqslant \kappa$, for some $\kappa>0$, holds. Let $A$ be the open (in $\bar{U}$ ) set defined by $A \equiv\|g\|^{-1}(d / 2,+\infty) \equiv\{x \in \bar{U}:\|g(x)\|>d / 2\}$. Clearly, $\bar{S} \cap \operatorname{supp} h \cap \partial U \subset$ $A$. Furthermore, $(\bar{S} \backslash A) \cap \operatorname{supp} h$ and $\partial U$ are disjoint closed sets and so, by normality, there exists an open set $V$ such that $(\bar{S} \backslash A) \cap \operatorname{supp} h \subset V \subset \bar{V} \subset U$. Then $V \cup A$ in an open (in $\bar{U}$ ) neighborhood of $\bar{S} \cap \operatorname{supp} h$ and, if necessary, intersecting it with a large ball containing $\bar{S} \cap \operatorname{supp} h$, we may assume that $V \cup A$ is bounded. Now, $(\bar{V} \cup \bar{A}) \cap \partial U=\bar{A} \cap \partial U$ and $\|g(\bar{A} \cap \partial U)\| \geqslant d / 2$. Set $W=(V \cup$ $A) \cap \operatorname{supp} h$. Clearly, $W$ is an open subset of $\operatorname{supp} h$ such that $\bar{S} \cap \operatorname{supp} h \subset W$ and $\operatorname{dist}(g(\bar{W} \cap \partial U), 0) \geqslant d / 2>0$. Since, $\bar{S} \cap \operatorname{supp} h$ and supp $h \backslash W$ are two closed disjoint subsets of supp $h$, there exists an Urysohn function $\phi$ with $\phi(\operatorname{supp} h \backslash W)=0$ and $\phi(\bar{S} \cap \operatorname{supp} h)=1$. Let $\tilde{h}$ be the map defined as $\phi h$ on $\operatorname{supp} h$ and zero on 
$E \backslash \operatorname{supp} h$. Clearly, $\{x \in E: \tilde{h}(x) \neq 0\} \subset W \cap\{x \in E: h(x) \neq 0\} \subset U$ and $\tilde{h}$ is compact since so is $h$. If $g(x)=\tilde{h}(x)$ for some $x \in S \cap U$, then either $x \in S \cap$ $\operatorname{supp} h$, in which case $\tilde{h}(x)=h(x)$, or $x \in U \backslash \operatorname{supp} h$ and $h(x)=\tilde{h}(x)=0$. One may thus replace $h$ by $\tilde{h}$ and assume that $\operatorname{dist}(g(\operatorname{supp} h \cap \partial U), 0) \geqslant \kappa>0$, where $\kappa=d / 2$.

Our next step will be to replace our new function $h$ with another having support contained in $U$. To do this consider the open (in $\bar{U}$ ) sets

$$
\bar{A}_{\kappa / 2}=\|g\|^{-1}(\kappa / 2,+\infty), \quad B_{\kappa / 2}=\|h\|^{-1}([0, \kappa / 3)) \cap \bar{U},
$$

both of which contain supp $h \cap \partial U$, and the closed sets

$$
\bar{A}_{3 \kappa / 4}=\|g\|^{-1}([3 \kappa / 4,+\infty)), \quad \bar{B}_{\kappa / 6}=\|h\|^{-1}([0, \kappa / 6]),
$$

also containing supp $h \cap \partial U$. Then, the set $A_{\kappa / 2} \cap B_{\kappa / 3}$ is open (in $\bar{U}$ ) and the set $\bar{A}_{3 \kappa / 4} \cap \bar{B}_{\kappa / 6}$ is closed and contained in the first. Clearly, supp $h \backslash\left(A_{\kappa / 2} \cap B_{\kappa / 3}\right)$ and $\bar{A}_{3 \kappa / 4} \cap \bar{B}_{\kappa / 6} \cap \operatorname{supp} h$ are two closed and disjoint subset of supp $h$ so that there is an Urysohn function $\varphi: \operatorname{supp} h \rightarrow[0,1]$ such that $\varphi\left(\bar{A}_{3 \kappa / 4} \cap \bar{B}_{\kappa / 6} \cap \operatorname{supp} h\right)=0$ and $\varphi\left(\operatorname{supp} h \backslash\left(A_{\kappa / 2} \cap B_{\kappa / 3}\right)\right)=1$. Let us put $\tilde{h}(x)=\varphi(x) h(x)$; then $\operatorname{supp} \tilde{h} \subset$ $\operatorname{supp} \varphi \subset \operatorname{supp} h \backslash\left(A_{3 \kappa / 4} \cap B_{\kappa / 6}\right) \subset U$. Finally, $g(x)=\tilde{h}(x)$ has a solution $\bar{x} \in S$ $\cap U$. However, on $A_{\kappa / 2} \cap B_{\kappa / 3} \cap S$, one has $\|g(x)\|>\kappa / 2,\|h(x)\|<\kappa / 3$ and $\|\tilde{h}(x)\|<\kappa / 3$, so that $\bar{x} \notin A_{\kappa / 2} \cap B_{\kappa / 3} \cap S$. This implies that $g(\bar{x})=h(\bar{x})$. Q.E.D.

Property 2.8. Assume that $A$ is a closed (in $U$ ) subset of $U$ such that $\bar{S} \cap A=\varnothing$. Then $g$ is 0-epi on $S \cap(U \backslash A)(S \cap(\overline{U \backslash A}))$ if and only if $g$ is 0-epi on $S \cap U$ $(S \cap \bar{U})$.

Proof. (If). This will follow from Property 2.2 provided $g$ is admissible on $S \cap(U \backslash A)(S \cap(\overline{U \backslash A}))$. Now, since $\bar{S} \cap A=\varnothing$, there exists an open subset $W$ such that $A \subset W \subset \bar{W} \subset U$ and $\bar{S} \cap \bar{W} \cap U=\varnothing$. Then $g^{-1}(0) \cap S \cap U=g^{-1}(0)$ $\cap S \cap(U \backslash A)$ is bounded. On the other hand, $g^{-1}(0) \cap S \cap U=g^{-1}(0) \cap S \subset$ $U \backslash \bar{W} \subset U \backslash W \subset U \backslash A$ (notice that $U \backslash W$ is closed in $U$ and in $U \backslash A$ ). Moreover, since $g$ is admissible on $S \cap U$, there exists an open and bounded subset $V_{0}$ such that $g^{-1}(0) \cap S \subset V_{0} \subset \bar{V}_{0} \subset U$. Therefore, $g^{-1}(0) \cap S \subset V_{0} \cap(U \backslash \bar{W}) \subset V_{0}$ $\cap W^{c} \subset U \backslash A$.

(Only if). Let $h: E \rightarrow G$ be compact with bounded support contained in $U$. If supp $h \subset U \backslash A$, then $g(x)=h(x)$ has a solution in $S \cap(U \backslash A)=S \cap U$. If supp $h$ $\cap A \neq \varnothing$, take an Urysohn function $\varphi: E \rightarrow[0,1]$ such that $\varphi(x)=1$ on $\bar{S}$ and $\varphi(x)=0$ on $\bar{W}$, where $\bar{W}$ is closed and $\operatorname{supp} h \cap A \subset W \subset \bar{W} \subset U$ (notice that supp $h \cap A$ is closed in $E)$. Let $\tilde{h}: E \rightarrow G$ be defined by $\tilde{h}(x)=\varphi(x) h(x)$. We have $\{x \in E: \tilde{h}(x) \neq 0\}=\{x \in E: h(x) \neq 0\} \cap\{x \in E: \varphi(x) \neq 0\}$. Therefore, supp $\tilde{h}$ $\subset \operatorname{supp} h \cap \operatorname{supp} \varphi \subset U \backslash A$. Hence, $g(x)=\tilde{h}(x)$ has a solution in $S \cap(U \backslash A)=$ $S \cap U$, where $\varphi \equiv 1$. Now, assume that $g$ is 0 -epi on $S \cap(\overline{U \backslash A})$. If $\{x \in E$ : $h(x) \neq 0\} \subset U \backslash A$, then $g(x)=h(x)$ has a solution in $S \cap(U \backslash A)=S \cap U$. If $\{x \in E: h(x) \neq 0\} \cap A \neq \varnothing$, then supp $h \cap A \neq \varnothing$ and is closed in $U$. It follows that there exists an open subset $W$ such that supp $h \cap A \subset W \subset \bar{W} \cap U, \bar{W} \cap U \cap$ $\bar{S}=\varnothing$. Let $\varphi: U \rightarrow[0,1]$ be an Urysohn function such that $\varphi(x)=1$ on $\bar{S}$ and $\varphi(x)=0$ on $\bar{W} \cap U$. Define $\tilde{h}: E \rightarrow G$ by $\tilde{h}(x)=\varphi(x) h(x)$ on $U$ and $\tilde{h}(x)=0$ on 
$U^{c}$ (notice that $\tilde{h}$ is continuous since $h(x)=0$ on $\partial U$ ). Hence, $\{x \in E: \tilde{h}(x) \neq 0\}$ $\subset\{x \in U: \varphi(x) \neq 0\} \cap\{x \in U: h(x) \neq 0\} \subset U \backslash A$ and we are done. Q.E.D.

EXAMPLE 2.1. Let $V \subset U$ be an open neighborhood of $\bar{S} \cap U$. Take $A=V^{c}$. Then, on the basis of Property 2.8, we have that $g$ is 0 -epi on $S \cap V(S \cap \bar{V})$ if and only if $g$ is 0 -epi on $S \cap U(S \cap \bar{U})$.

The following simple, though important, property will be used several times in the sequel.

Property 2.9. Let $G_{i}, i=1,2$, be Banach spaces and let $g_{i}: U \rightarrow G_{i}, i=1,2$, be continuous maps. Define $g: U \rightarrow G_{1} \times G_{2}$ by $g(x)=\left(g_{1}(x), g_{2}(x)\right)$. Assume that $g$ is 0 -epi on $S \cap U(S \cap \bar{U})$. Then $g_{2}$ is 0-epi on $g_{1}^{-1}(0) \cap S \cap U\left(g_{1}^{-1}(0) \cap S \cap \bar{U}\right)$.

Proof. If $h_{2}: E \rightarrow G_{2}$ is compact with bounded support contained in $U$ ( $\bar{U}$ and $h_{2}(x)=0$ on $\left.\partial U\right)$, then the map $h \equiv\left(0, h_{2}\right)$ is compact on $E$ and has bounded support contained in $U(\bar{U}$ and $h(x)=0$ on $\partial U)$. Therefore, the equations $g_{2}(x)=$ $h_{2}(x)$ and $g_{1}(x)=0$ are solvable in $S \cap U$. Q.E.D.

Note that if $S$ is closed in $U\left(S \cap \bar{U}\right.$ is closed), then $g^{-1}(0) \cap S$ is closed in $U$ $\left(g^{-1}(0) \cap S \cap \bar{U}\right.$ is closed).

The examples given in the Introduction show that $g_{2}$ may be 0 -epi on $g_{1}^{-1}(0) \cap S$ $\cap U$ and, nevertheless, the map $g=\left(g_{1}, g_{2}\right)$ may be not 0 -epi on $S \cap U$.

C. Homotopy property. Up to this point we did not impose any assumption on the set $S$. In order to get deeper results we shall assume that $S$ is closed in $U$ for 0 -epi maps on $S \cap U$ and assume $S \cap \bar{U}$ closed in $E$ when considering 0-epi maps on $S \cap \bar{U}$. The fact that $S$ need not be closed (nor open) for a map $g$ to be 0 -epi on $S \cap U$ can be seen from the following example. Take $U=\mathbf{R}^{2}, S=\{y$-axis $\} \cup \kappa$, where $\kappa$ is any set not intersecting the $x$-axis, and define $g: \mathbf{R}^{2} \rightarrow \mathbf{R}$ by $g(x, y)=y$. Then $g$ is 0 -epi on $S \cap U$. To see this take a sufficiently large ball $B$ centered at the origin. Then, $g(S \cap \partial B) \neq 0$ by the choice of $\kappa$ and, if $\tilde{g}$ is a nonvanishing (on $S$ ) extension of $\left.g\right|_{S \cap \partial B}$ to $S \cap \bar{B}$, then $\tilde{g}(0, y) \neq 0$ on the $y$-axis, which is impossible since $\left.g\right|_{\{y \text {-axis }\} \cap \partial B}$ changes sign. Due to the fact that any bounded set may be included in such a ball, from Proposition 2.1 and Property 2.2 it follows that $g$ is 0 -epi on $S \cap U$.

Finally, if $g$ is 0-epi on $S \cap U(S \cap \bar{U})$ and $g$ is admissible on $\bar{S} \cap U(\bar{S} \cap \bar{U})$, then, from Property 2.5, we obtain that $g$ is 0 -epi on $\bar{S} \cap U(\bar{S} \cap \bar{U})$. This shows, in a certain sense, that the requirement of the closedness of $S$ is not too heavy.

Our homotopy principle will be based on the following result, which is, in its turn, an extension of [FMV,I, Theorem 4.2.1].

Theorem 2.1 (COINCIDENCE THEOREM). Let $g: U \rightarrow G$ be 0 -epi on $S \cap U$, where $S$ is closed in $U$. Let $h: U \rightarrow G$ be continuous and such that:

(1) There exists an open and bounded subset $V_{0}$ with the property

$$
A \equiv\{x \in S \cap U: g(x)-\operatorname{th}(x)=0 \text { for some } t \in[0,1]\} \subset V_{0} \subset \bar{V}_{0} \subset U \text {. }
$$

(2) Let $\varphi: E \rightarrow[0,1]$ be a given continuous function such that its support (denoted by $\left.\bar{V}_{1}\right)$ is bounded and contained in $U$, and $\varphi\left(\bar{V}_{0}\right)=1$. Assume that the following holds: if $M$ is a bounded and closed (in $E$ ) subset such that

$$
M=g^{-1}(\overline{\operatorname{co}}((\varphi h)(M) \cup\{0\})) \cap S \cap \bar{V}_{1},
$$

then $(\varphi h)(M)$ is precompact. 
Then the equation $g(x)=h(x)$ has a solution in $S \cap U$. (If $\operatorname{supp} h$ is bounded and contained in $U$, then the set $A$ satisfies automatically condition (1). It suffices to take $V_{0} \supset\left(g^{-1}(0) \cap S\right) \cup \operatorname{supp} h$. By admissibility this set is always bounded, contained in $U$ and one has $\varphi h=h$.)

(In the case when $g: \bar{U} \rightarrow G$ is 0 -epi on the closed set $S \cap \bar{U}$ and $A \subset V_{0} \subset U$, one gets the same conclusion.)

Proof. Set $\tilde{h}=\varphi h$. If there is some $\bar{x} \in S \cap U$ such that $g(\bar{x})=\varphi(\bar{x}) h(\bar{x})$, then $\bar{x} \in S \cap V_{0}$ and $\varphi(\bar{x})=1$. Hence $g(\bar{x})=h(\bar{x})$. Let $\mathscr{M}$ be the following family of sets:

$$
\mathscr{M}=\left\{M \subset S \cap \bar{V}_{1}: M \text { is closed and } g^{-1}(\overline{\operatorname{co}}(\tilde{h}(M) \cup\{0\})) \cap S \cap \bar{V}_{1} \subset M\right\} .
$$

The family $\mathscr{M}$ is not empty since $S \cap \bar{V}_{1} \in \mathscr{M}$. If $M \in \mathscr{M}$, then $g^{-1}(0) \cap S \cap \bar{V}_{1}=$ $g^{-1}(0) \cap S \subset M$ (recall that $g^{-1}(0) \cap S$ is not empty, since $g$ is 0 -epi on $S \cap U$ ). Let $M_{0}=\bigcap_{M \in \mathscr{M}} M ;$ then $M_{0} \supset g^{-1}(0) \cap S \neq \varnothing$. Now,

$$
g^{-1}\left(\overline{\operatorname{co}}\left(\tilde{h}\left(M_{0}\right) \cup\{0\}\right)\right) \cap S \cap \bar{V}_{1} \subset g^{-1}(\overline{\operatorname{co}}(\tilde{h}(M) \cup\{0\})) \cap S \cap \bar{V}_{1}
$$

for all $M \in \mathscr{M}$.

This implies that

$$
M_{1} \equiv g^{-1}\left(\overline{\operatorname{co}}\left(\tilde{h}\left(M_{0}\right) \cup\{0\}\right)\right) \cap S \cap \bar{V}_{1} \subset M_{0} .
$$

Moreover, $M_{1}$ is closed. Therefore,

$$
g^{-1}\left(\overline{\operatorname{co}}\left(\tilde{h}\left(M_{1}\right) \cup\{0\}\right)\right) \cap S \cap \bar{V}_{1} \subset g^{-1}\left(\overline{\operatorname{co}}\left(\tilde{h}\left(M_{0}\right) \cup\{0\}\right)\right) \cap S \cap \bar{V}_{1}=M_{1} \text {. }
$$

It follows that $M_{1}=M_{0}$ and, by condition (2), we obtain that $\tilde{h}\left(M_{0}\right)$ is precompact. Now, define $\hat{h}$ as $\tilde{h}$ on $M_{0}$ and zero on $V_{1}^{c}$. Clearly, $\hat{h}$ is a compact map and, by Dugundji's extension theorem, there exists a compact extension

$$
\check{h}: E \rightarrow \overline{\operatorname{co}}\left(\tilde{h}\left(M_{0}\right) \cup\{0\}\right)
$$

of $\hat{h}$ such that supp $\check{h} \subset \bar{V}_{1} \subset U$. Since $g$ is 0-epi on $S \cap U$, there exists $x_{0} \in S \cap U$ such that $g\left(x_{0}\right)=\breve{h}\left(x_{0}\right)$. Since $\breve{h}\left(x_{0}\right) \in \overline{c o}\left(\tilde{h}\left(M_{0}\right) \cup\{0\}\right)$, we get $x_{0} \in$ $g^{-1}\left(\overline{\operatorname{co}}\left(\tilde{h}\left(M_{0}\right) \cup\{0\}\right)\right) \cap S$. Moreover, $x_{0} \in V_{1}$. Indeed, if $x_{0} \in V_{1}^{c}$, then $\varphi\left(x_{0}\right)=0$ $=\hat{h}\left(x_{0}\right)$, yielding $g\left(x_{0}\right)=0$. Then, $x_{0} \in g^{-1}(0) \cap S \subset V_{0} \subset V_{1}$, and therefore $x_{0} \in$ $M_{0}, \hat{h}\left(x_{0}\right)=\tilde{h}\left(x_{0}\right)$, which gives $g\left(x_{0}\right)=\varphi\left(x_{0}\right) h\left(x_{0}\right)=h\left(x_{0}\right)$. Q.E.D.

Note that in Theorem 2.1 one could take the function $\varphi$ with $\varphi(A)=1$ and $\varphi\left(V_{0}^{c}\right)=0$.

The following theorem is a further important property of 0 -epi maps on $S \cap U$ $(S \cap \bar{U})$. Its proof will rely on Theorem 2.1 .

THEOREM 2.2 (Homotopy PRINCIPLE). Let $g: U \rightarrow G$ be 0 -epi on $S \cap U$ and let $h$ : $(S \cap U) \times[0,1] \rightarrow G$ be continuous and such that $h(x, 0)=0$ on $S \cap U$. Assume that the following two assumptions hold:

(1) There exists an open and bounded set $V_{0}$ for which $A_{0} \equiv\{x \in S \cap U: g(x)-$ $h(x, t)=0$ for some $t \in[0,1]\} \subset V_{0} \subset \bar{V}_{0} \subset U$. 
(2) For any compact map $k: E \rightarrow G$ with bounded support contained in $U$ set $A_{k} \equiv\{x \in S \cap U: g(x)-h(x, t)-k(x)=0$ for some $t \in[0,1]\}$ and let $\varphi_{k}: E \rightarrow$ $[0,1]$ be a continuous function such that $\varphi_{k}\left(A_{k}\right)=1$ and $\varphi_{k}\left(V_{k}^{c}\right)=0$, where $V_{k}=V_{0}$ $\cup\{x \in E: k(x) \neq 0\}$. Assume the following: If $M$ is a closed subset of $S \cap \bar{V}_{k}$ such ihat $M=g^{-1}\left(\overline{\operatorname{co}}\left(h\left(\left(\cdot, \varphi_{k}(\cdot)\right)+k(\cdot)\right)(M) \cup\{0\}\right)\right) \cap S \cap \bar{V}_{k}$, then $h\left(\cdot, \varphi_{k}(\cdot)\right)(M)$ is precompact.

Then, the map $g(\cdot)+\tilde{h}(\cdot)$ is 0-epi on $S \cap U$ for any $\tilde{h}$ continuous extension to $U$ of $h(\cdot, 1)$. (If $g: \bar{U} \rightarrow G$ is 0 -epi on $S \cap \bar{U}$, replace $U$ everywhere by $\bar{U}$ and recall that, in this case, $k(x)=0$ on $\partial U$.)

Proof. The first step of the proof will be to extend $h$ to a continuous map $\tilde{h}$ defined on $U \times[0,1]$ with $\tilde{h}(x, 0)=0$ and $\tilde{h}(x, 1)=\tilde{h}(x)$ on $U$. This is done via Dugundji's extension theorem since $((S \cap U) \times[0,1]) \cup(U \times\{0\}) \cup(U \times\{1\})$ is a closed subset of $U \times[0,1]$.

Now, the inclusion $A_{k} \subset\{x \in E: k(x) \neq 0\} \cup A_{0} \subset \operatorname{supp} k \cup V_{0} \subset U$ holds, so that the set $A_{k}$ is bounded. Let us show that $A_{k}$ is also closed. Take a sequence $\left\{x_{n}\right\}$, $x_{n} \in A_{k}, n \in \mathbf{N}$, such that $x_{n} \rightarrow x$. There corresponds a sequence $\left\{t_{n}\right\}$ in $[0,1]$ such that $g\left(x_{n}\right)-h\left(x_{n}, t_{n}\right)=k\left(x_{n}\right)$. Without loss of generality we may assume that $t_{n}$ converges to some element $t \in[0,1]$. Taking into account the continuity of all the maps involved and the fact that $S$ is closed in $U$, we obtain that $x \in A_{k}$, i.e., $A_{k}$ is closed (notice that the same argument yields the closedness of $A_{0}$ ). Let $\varphi_{k}$ be an Urysohn function such that $\varphi_{k}\left(A_{k}\right)=1$ and $\varphi_{k}\left(V_{k}^{c}\right)=0$. Now, consider the map $\tilde{h}\left(\cdot, \varphi_{k}(\cdot)\right)+k(\cdot)$ which is identically zero on $V_{k}^{c}$ and coincides with the map $h\left(\cdot, \varphi_{k}(\cdot)\right)+k(\cdot)$ on $S \cap \bar{V}_{k}$. Observe that if $g(x)=h\left(x, \varphi_{k}(x)\right)+k(x)$ for some $x \in S \cap U$, then $\varphi_{k}(x)=1$ (since $x \in A_{k}$ ) and, by Theorem 2.1, one has that $g-\tilde{h}$ is 0 -epi on $S \cap U$ (recall that our perturbation has bounded support). Q.E.D.

The following consequences of Theorem 2.2 will show that condition (2) covers several well-known cases of homotopy invariance.

Corollary 2.1. (a) Assume that the homotopy $h(\cdot, \cdot)$ of Theorem 2.2 is compact on $(S \cap U) \times[0,1]$. Then condition (2) is satisfied.

(b) Let $g: \bar{U} \rightarrow G$ be 0-epi on the bounded set $S \cap \bar{U}$ and let $h:(S \cap \partial U) \times[0,1]$ $\rightarrow G$ be compact and $h(x, 0)=0$ on $S \cap \partial U$. Assume that $g(x) \neq h(x, t)$ on $(S \cap$ $\partial U) \times[0,1]$. Then, $g-\tilde{h}$ is 0-epi on $S \cap \bar{U}$ for any extension $\tilde{h}$ to $\bar{U}$ of $h(\cdot, 1)$ which is compact on $S \cap \bar{U}$.

Proof. (a) Obviously, condition (2) is satisfied. We would like to mention the fact that one can give a direct proof of this part of the proof by observing that the map $h\left(\cdot, \varphi_{k}(\cdot)\right)+k(\cdot)$ is compact and with bounded support contained in $U$. Thus, from the definition of 0 -epi map on $S \cap U$, the result follows.

(b) Let $\breve{h}$ be the compact map defined as zero on $(S \cap \bar{U}) \times\{0\}, h$ on $(S \cap \partial U)$ $\times[0,1]$ and $\tilde{h}$ on $(S \cap \bar{U}) \times\{1\}$. By Dugundji's extension theorem $\check{h}$ can be compactly extended to a map $\tilde{h}$ defined on $(S \cap \bar{U}) \times[0,1]$. We shall show that the set $A_{0} \equiv\{x \in S \cap \bar{U}: g(x)-\tilde{h}(x, t)=0$ for some $t \in[0,1]\}$ is a bounded subset of $U$. Indeed, notice first that $A_{0} \subset U$, since if $g(x)-\tilde{h}(x, t)=0$ for some $x \in S \cap$ $\partial U$ and $t \in[0,1]$, then $\tilde{h}=h$. But $g(x)-h(x, t) \neq 0$ and thus $A_{0} \cap \partial U=\varnothing$. 
Secondly, $A_{0}$ is bounded since so is $S \cap \bar{U}$. Therefore, by part (a) above the map $g(\cdot)-\tilde{h}(\cdot, 1)$ is 0 -epi on $S \cap \bar{U}$. Q.E.D.

REMARK 2.8. Note that Corollary $2.1(\mathrm{~b})$ is very close in spirit to the classical Borsuk extension theorem in finite dimensions. In fact,

Let $X$ be a metric space, $A$ a closed subset of $X$ and $g: X \rightarrow G$ a continuous map. Let $h: A \times[0,1] \rightarrow G$ be a compact map such that $h(x, 0)=0$ on $A$ and $g(x)+h(x, t) \neq 0$ on $A \times[0,1]$. If the restriction $\left.g\right|_{A}$ has a nonvanishing extension $\tilde{g}$ to $X$ such that $g-\tilde{g}$ is compact on $X$, then $\left.\left(g+h_{1}\right)\right|_{A}$ has a nonvanishing extension $g+\tilde{h}_{1}$ on $X$ such that $\tilde{h}_{1}$ is compact on $X$.

Proof. (Sketch). The map $H:(X \times\{0\}) \cup(A \times[0,1]) \rightarrow G$ defined as $g-\tilde{g}$ on $X \times\{0\}$ and $h$ on $A \times[0,1]$ satisfies $g+H \neq 0$ on that set and, by Dugundji's extension theorem, it has a compact extension $\tilde{H}$ to $X \times[0,1]$. Now, the closed sets $A$ and $B=\{x \in X ; g(x)+\tilde{H}(x, t)=0$ for some $t \in[0,1]\}$ are disjoint. Hence, there is an Urysohn's function $\varphi: X \rightarrow[0,1]$ with $\varphi(B)=0$ and $\varphi(A)=1$. Finally, let $\hat{g}: X \times[0,1] \rightarrow G \backslash\{0\}$ be the map defined by $\hat{g}(x, t)=g(x)+\tilde{H}(x, t \varphi(x))$. Clearly, $\hat{g}_{0}=\tilde{g}$ and $\left.\hat{g}_{1}\right|_{A}=\left.\left(g+h_{1}\right)\right|_{A}$ so that $\hat{g}_{1}$ is the required extension. Q.E.D.

In the sequel the notation $\alpha(A)$ will stand for a measure of noncompactness of the set $A$, satisfying the following properties.

(1) $\alpha(A)=0$ if and only if $\bar{A}$ is compact,

(2) $\alpha(A \cup B)=\max \{\alpha(A), \alpha(B)\}$ (this implies $\alpha(A) \leqslant \alpha(B)$ if $A \subset B$ ),

(3) $\alpha(\overline{\operatorname{co}} A)=\alpha(A)$,

(4) $\alpha(A+B) \leqslant \alpha(A)+\alpha(B)$,

(5) $\alpha(t A)=t \alpha(A)$ for any $t \geqslant 0$.

DeFinition 2.4. Let the maps $g: S \cap U \rightarrow G$ and $h:(S \cap U) \times[0,1] \rightarrow G$ send bounded and closed (in $E$ ) sets into bounded sets. The map $h$ will be called condensing with respect to $g$ on $S \cap U$ if and only if for any bounded set $M \subset \bar{M} \subset S$ $\cap U$ we have

$$
\alpha(h(\cdot, \cdot)(M \times[0,1]))<\alpha(g(M))
$$

if $\alpha(g(M))>0$ and

$$
\alpha(h(\cdot, \cdot)(M \times[0,1]))=0
$$

if $\alpha(g(M))=0$. (A similar definition holds in the case when $U$ is replaced by $\bar{U}$.)

COROLlary 2.2. If $h$ is condensing with respect to $g$ on $S \cap U(S \cap \bar{U})$, then condition (2) of Theorem 2.2 is satisfied.

Proof. Let $M$ be a closed subset of $S \cap V_{k}$, satisfying the assumption of condition (2). Then

$$
g(M) \subset \overline{\operatorname{co}}\left(\left(h\left(\cdot, \varphi_{k}(\cdot)\right)+k(\cdot)\right)(M) \cup\{0\}\right) .
$$

By properties (1)-(5) of the measure of noncompactness $\alpha$ we get

$$
\alpha(g(M)) \leqslant \alpha\left(h\left(\cdot, \varphi_{k}(\cdot)\right)(M)\right) \leqslant \alpha(h(\cdot, \cdot)(M \times[0,1])) .
$$

This may occur only if $\alpha(h(\cdot, \cdot)(M \times[0,1]))=0$, since $h$ is condensing with respect to $g$ on $S \cap U$. Q.E.D. 
Definition 2.5. Let $g \equiv f-g_{0}: S \cap U \rightarrow G$ and $h:(S \cap U) \times[0,1] \rightarrow G$ be such that $f, g_{0}$ and $h$ send bounded and closed (in $E$ ) sets into bounded sets. The homotopy $g_{0}(\cdot)+h(\cdot, \cdot)$ is called $\kappa$-set-contractive with respect to $f$ on $S \cap U$ if and only if there exists $0 \leqslant \kappa<1$ such that for any bounded subset $M \subset \bar{M} \subset S \cap U$ the following holds:

$$
\alpha\left(\left(g_{0}(\cdot)+h(\cdot, \cdot)\right)(M \times[0,1])\right) \leqslant \kappa \alpha(f(M)) .
$$

(An analogous definition holds in the $\bar{U}$ case.)

Corollary 2.3. Let $g \equiv f-g_{0}: U \rightarrow G$ be 0-epi on $S \cap U$ and let $g_{0}+h$ be $\kappa$-set-contractive with respect to $f$ on $S \cap U$, with $h(x, 0)=0$ on $S \cap U$. Assume that there exists an open and bounded set $V_{0}$ such that $A_{0} \equiv\left\{x \in S \cap U: f(x)-g_{0}(x)-\right.$ $h(x, t)=0$ for some $t \in[0,1]\} \subset V_{0} \subset \bar{V}_{0} \subset U$. Then $f(\cdot)-g_{0}(\cdot)-\tilde{h}(\cdot)$ is 0-epi on $S \cap U$ for any $\tilde{h}$ continuous extension to $U$ of $h(\cdot, 1)$.

Proof. The starting point is to make the same extensions as in the first step of the proof of Theorem 2.2. Next, let $V$ be any bounded open set such that $A_{0} \subset V \subset \bar{V}$ $\subset U$. Let $\varphi: E \rightarrow[0,1]$ be an Urysohn's function, having bounded support contained in $U$, and such that $\varphi(\bar{V})=1$. Consider the map $f(\cdot)-g_{0}(\cdot)-\tilde{h}(\cdot, t \varphi(\cdot))=$ $g(\cdot)-\tilde{h}_{\varphi}(\cdot, t)$. Clearly, $h_{\varphi}$ has bounded support, so that condition (1) of Theorem 2.2 is automatically satisfied by $h_{\varphi}$ and by $\tau h_{\varphi}$ for any $\tau \in \mathbf{R}$. We shall prove that the map $g(\cdot)-\tilde{h}(\cdot, \varphi(\cdot))$ is 0-epi on $S \cap U$. Since the zeros of this map on $S \cap U$ are in $A_{0} \subset V$, then $g(\cdot)-\tilde{h}(\cdot, \varphi(\cdot))$ is 0-epi on $S \cap \bar{V}$. But $\varphi(\bar{V})=1$ and hence the map $g(\cdot)-\tilde{h}(\cdot)$ is 0 -epi on $S \cap \bar{V}$ for any such $V$. Hence, by definition, $g(\cdot)-\tilde{h}(\cdot)$ is 0 -epi on $S \cap U$. Now,

$$
\begin{aligned}
& \alpha\left(\left(g_{0}(\cdot)+\tilde{h}(\cdot, \cdot \varphi(\cdot))\right)(M \times[0,1])\right) \\
& \quad \leqslant \alpha\left(\left(g_{0}(\cdot)+\tilde{h}(\cdot, \cdot)\right)(M \times[0,1])\right) \leqslant \kappa \alpha(f(M)) .
\end{aligned}
$$

This implies, $\alpha\left(\left(g_{0}(\cdot)+\tilde{h}(\cdot, t \varphi(\cdot))\right)(M)\right) \leqslant \kappa \alpha(f(M))$ for all $t \in[0,1]$ (notice, for $t=0$, we have $\left.\alpha\left(g_{0}(M)\right) \leqslant \kappa \alpha(f(M))\right)$. The trivial equality $\tilde{h}(\cdot, \cdot \varphi(\cdot))=g_{0}(\cdot)+$ $\tilde{h}(\cdot, \cdot \varphi(\cdot))-g_{0}(\cdot)$ yields

$$
\alpha((\tilde{h}(\cdot, \cdot \varphi(\cdot)))(M \times[0,1])) \leqslant 2 \kappa \alpha(f(M)) .
$$

Also, the equality $f(\cdot)=f(\cdot)-g_{0}(\cdot)-\tilde{h}(\cdot, t \varphi(\cdot))+g_{0}(\cdot)+\tilde{h}(\cdot, t \varphi(\cdot))$ gives

$$
\alpha(f(M)) \leqslant \alpha\left(\left(f(\cdot)-g_{0}(\cdot)-\tilde{h}(\cdot, t \varphi(\cdot))\right)(M)\right)+\kappa \alpha(f(M)) .
$$

Thus,

$$
(1-\kappa) \alpha(f(M)) \leqslant \alpha\left(\left(f(\cdot)-g_{0}(\cdot)-\tilde{h}(\cdot, t \varphi(\cdot))\right)(M)\right) .
$$

Now, for $t=0$

$$
\begin{aligned}
\alpha((\tau \tilde{h}(\cdot, \cdot \varphi(\cdot)))(M \times[0,1])) & \leqslant 2 \kappa \tau \alpha(f(M)) \\
& \leqslant \frac{2 \kappa \tau}{1-k} \alpha\left(\left(f(\cdot)-g_{0}(\cdot)\right)(M)\right) .
\end{aligned}
$$


Therefore, by Corollary 2.2, the map $f(\cdot)-g_{0}(\cdot)-\tau \tilde{h}(\cdot, \varphi(\cdot))$ is 0 -epi on $S \cap U$ provided $\tau<(1-\kappa) / 2 \kappa$. Now, this map can be written as

$$
f(\cdot)-\left(\tau\left(g_{0}(\cdot)+\tilde{h}(\cdot, \varphi(\cdot))\right)+(1-\tau) g_{0}(\cdot)\right) .
$$

But,

$$
\begin{aligned}
& \alpha\left(\left(\tau\left(g_{0}(\cdot)+\tilde{h}(\cdot, \varphi(\cdot))\right)+(1-\tau) g_{0}(\cdot)\right)(M)\right) \\
& \quad \leqslant \tau \kappa \alpha(f(M))+(1-\tau) \kappa \alpha(f(M))=\kappa \alpha(f(M)) .
\end{aligned}
$$

Therefore, as above,

$$
(1-\kappa) \alpha(f(M)) \leqslant \alpha\left(\left(f(\cdot)-\left(\tau\left(g_{0}(\cdot)+\tilde{h}(\cdot, \varphi(\cdot))\right)+(1-\tau) g_{0}(\cdot)\right)\right)(M)\right) .
$$

Again, if $\tau<(1-\kappa) / 2 \kappa$, then the map $f(\cdot)-g_{0}(\cdot)-2 \tau \tilde{h}(\cdot, \varphi(\cdot))$ is 0 -epi on $S \cap U$. In a finite number of steps one gets to $f(\cdot)-g_{0}(\cdot)-\tilde{h}(\cdot, \varphi(\cdot))$, which is 0 -epi on $S \cap U$. Q.E.D.

We would like to remark that Corollary 2.3 is an extension of Theorem 7.3.1 of [FMV,I].

Corollary 2.4. Let $g \equiv f-g_{0}: U \rightarrow G$ be 0-epi on $S \cap U$ and let $h:(S \cap U) \times$ $[0,1] \rightarrow G$ be such that the map $g_{0}(\cdot)+h(\cdot, \cdot)$ is condensing with respect to $f$ on $S \cap U$. Assume that $h(x, 0)=0$ on $S \cap U$ and let $A_{0}$ be as in Corollary 2.3. Furthermore, suppose that $f$ is proper on bounded and closed subsets of $S \cap U$ and that for any open and bounded subset $V$ such that $A_{0} \subset V \subset \bar{V} \subset U$ there exists $\tau_{V}$ for which the map $f-(1-\tau) g_{0}$ is 0 -epi on $S \cap \bar{V}$ for $0 \leqslant \tau<\tau_{V}$. Then the map $f(\cdot)-g_{0}(\cdot)-\tilde{h}(\cdot)$ is 0 -epi on $S \cap U$ for any $\tilde{h}$ continuous extension to $U$ of $h(\cdot, 1)$. ( $A$ similar result holds for the $\bar{U}$ case.)

Proof. Let $V$ be as above and let us show that there exists $\varepsilon>0$ such that $\left\|f(x)-g_{0}(x)-\tilde{h}(x, t)\right\| \geqslant \varepsilon$ for all $x \in S \cap \partial V$ and $t \in[0,1]$. In fact, if not, there is a sequence $\left\{\left(x_{n}, t_{n}\right)\right\}$ in $(S \cap \partial V) \times[0,1]$ such that $f\left(x_{n}\right)-g_{0}\left(x_{n}\right)-\tilde{h}\left(x_{n}, t_{n}\right)$ $\rightarrow 0$ as $n \rightarrow+\infty$. From the equality

$$
f\left(x_{n}\right)=g_{0}\left(x_{n}\right)+\tilde{h}\left(x_{n}, t_{n}\right)+\left(f\left(x_{n}\right)-g_{0}\left(x_{n}\right)-\tilde{h}\left(x_{n}, t_{n}\right)\right)
$$

it follows, denoting by $A$ the sequence $\left\{x_{n}\right\}$,

$$
\alpha(f(A)) \leqslant \alpha\left(\left(g_{0}(\cdot)+\tilde{h}(\cdot, \cdot)\right)(A \times[0,1])\right)<\alpha(f(A))
$$

which is impossible unless $f(A)$ is precompact. This implies, by the properness of $f$, that $\bar{A}$ is compact. Therefore, there exist subsequences $x_{n_{\kappa}} \rightarrow x$ and $t_{n_{\kappa}} \rightarrow t$ such that $f(x)-g_{0}(x)-\tilde{h}(x, t)=0$ for $x \in S \cap \partial V$, contradicting the admissibility of the homotopy.

Since $g_{0}(\cdot)+\tilde{h}(\cdot, \cdot)$ is bounded on bounded sets of $(S \cap U) \times[0,1]$, then $\left\|g_{0}(x)+\tilde{h}(x, t)\right\| \leqslant L$ on $(S \cap \bar{V}) \times[0,1]$. Choose $\tau$ such that $\tau L<\varepsilon / 2$. Then,

$$
\left\|f(x)-(1-\tau)\left(g_{0}(x)+\tilde{h}(x, t)\right)\right\| \geqslant \frac{\varepsilon}{2} \quad \text { on }(S \cap \partial U) \times[0,1] .
$$

Furthermore,

$$
\alpha\left((1-\tau)\left(g_{0}(\cdot)+\tilde{h}(\cdot, \cdot)\right)(M \times[0,1])\right) \leqslant(1-\tau) \alpha(f(M)) .
$$


From Corollary 2.3 it follows that the map $f-(1-\tau) g_{0}$ is 0 -epi on $S \cap \bar{V}$ if and only if the map $f(\cdot)-(1-\tau)\left(g_{0}(\cdot)+\tilde{h}(\cdot, \cdot)\right)$ is 0-epi on $S \cap \bar{V}$. Moreover, $f(\cdot)=$ $f(\cdot)-(1-\tau) g_{1}(\cdot)+(1-\tau) g_{1}(\cdot)$, where $g_{1}(\cdot)=g_{0}(\cdot)+\tilde{h}(\cdot, 1)$. This implies

$$
f(M) \subset\left(f(\cdot)-(1-\tau) g_{1}(\cdot)\right)(M)+(1-\tau) g_{1}(M) .
$$

Thus,

$$
\begin{aligned}
\alpha(f(M)) & \leqslant \alpha\left(\left(f(\cdot)-(1-\tau) g_{1}(\cdot)\right)(M)\right)+(1-\tau) \alpha\left(g_{1}(M)\right) \\
& <\alpha\left(\left(f(\cdot)-(1-\tau) g_{1}(\cdot)\right)(M)\right)+(1-\tau) \alpha(f(M))
\end{aligned}
$$

(if $\alpha(f(M))>0)$. Hence, $\tau \alpha(f(M))<\alpha\left(\left(f(\cdot)-(1-\tau) g_{1}(\cdot)\right)(M)\right)$ and $\tau \alpha\left(g_{1}(M)\right)$ $<\alpha\left(\left(f(\cdot)-(1-\tau) g_{1}(\cdot)\right)(M)\right)$. Notice that, setting $[0,1] M=\{t x: t \in[0,1]$ and $x \in M\}$, we have $M \subset[0,1] M \subset \overline{\operatorname{co}}(M \cup\{0\})$. Hence, $\alpha([0,1] M)=\alpha(M)$. Now, if $k(x, t)$ is the homotopy $t \tau g_{1}(x)$, then

$$
\alpha((k(\cdot, \cdot))(M \times[0,1]))=\tau \alpha\left(g_{1}(M)\right)<\alpha\left(\left(f(\cdot)-(1-\tau) g_{1}(\cdot)\right)(M)\right) .
$$

Hence, from Corollary 2.2, it follows that the map $f-(1-\tau) g_{1}-\tau g_{1}$ is 0 -epi on $S \cap \bar{V}$ if $f-(1-\tau) g_{1}$ is 0 -epi on $S \cap \bar{V}$. Therefore, the assumption that $f-$ $(1-\tau) g_{0}$ is 0 -epi on $S \cap \bar{V}$ provided $\tau<\tau_{V}$ ends the argument. Q.E.D.

Note that, in the case when $S=E$ and when $I-g_{0}$ has a nonzero topological degree (as a condensing vector field), then also the vector field $I-(1-\tau) g_{0}$ has nonzero topological degree. This is, actually, the way in which the degree for condensing vector fields can be constructed (cf. [N]).

The following result, which is another consequence of Corollary 2.1(a), gives information on the structure of the set $S \cap U$ when there is a map which is 0-epi on it.

Proposition 2.4. Let $g$ be 0-epi on $S \cap U(S \cap \bar{U})$. Then:

(a) Either $S \cap \partial V \neq \varnothing$, or $g(S \cap \bar{V})=G$ for any open and bounded set $V$ such that $g^{-1}(0) \cap S \subset V \subset \bar{V} \subset U\left(g^{-1}(0) \cap S \subset V \subset U\right)$. In particular, if $g$ sends bounded and closed (in $E)$ subsets of $S \cap U(S \cap \bar{U})$ into bounded sets of $G$, then $S \cap \partial V \neq \varnothing$.

(b) Either $S \cap U$ is unbounded, or there exists $V$ as above such that $g(S \cap \bar{V})=G$ or $(\overline{S \cap U}) \cap \partial U \neq \varnothing$. In particular, if $S \cap \bar{U}$ is closed, then $S \cap \partial U \neq \varnothing$.

Proof. (a) Assume there is such a $V$ for which $S \cap \partial V=\varnothing$. Then, from Property 2.4 (Property 2.2), the map $g$ is 0 -epi on the closed and bounded set $S \cap \bar{V}$. Assume that there exists $p \in G$ such that $p \notin g(S \cap \bar{V})$ and let $h(x, t)=t p$. Clearly, the map $h$ satisfies the assumptions of Corollary 2.1(b) $(g(x) \neq h(x, t)$ on $S \cap \partial V=\varnothing)$ and therefore $g-\tilde{h}$ is 0 -epi on $S \cap \bar{V}$, where $\tilde{h} \equiv p$. The result now follows from Property 2.1 .

(b) If $(\overline{S \cap U}) \cap \partial U=\varnothing$ and $S \cap U$ is bounded, then by normality, there exists an open and bounded set $V$ such that $\overline{S \cap U} \subset V \subset \bar{V} \subset U$. Since $S \cap \partial V=\varnothing$, then, from part (a), we get $g(S \cap \bar{V})=G$.

Finally, if $S \cap \bar{U}$ is closed and $(\overline{S \cap U}) \cap \partial U \neq \varnothing$, then $(\overline{S \cap U}) \cap \partial U \subset S \cap \partial U$, so that $S \cap \partial U \neq \varnothing$. Q.E.D.

D. Zero-epi (zero-essential) maps on sections. In this part we shall introduce a class of maps which is larger than that of 0 -epi maps. This new family of maps will be of 
crucial importance in proving results when the parameter space is infinite dimensional. Actually, our main result on dimension will be given for this broader class of maps.

Our starting point will be close to that adopted in [AA,II]. Let us first fix a family of sections for the Banach space $G$ in the following sense. Let $M_{0}$ be a fixed finite dimensional subspace of $G$ and let $\mathscr{M}$ be a collection of finite dimensional subspaces of $G$ satisfying the following conditions:

(i) If $M \in \mathscr{M}$, then $M_{0} \subset M$.

(ii) For all $M_{1}, M_{2} \in \mathscr{M}$ there exists $M_{3} \in \mathscr{M}$ such that $M_{1}, M_{2} \subset M_{3}$.

(iii) $\bar{\cup}_{M \in \mathscr{M}} \bar{M}=G$.

The family of sections of $G$ just defined differs from that introduced in $[\mathbf{A A}, \mathbf{I I}]$, where our condition (iii) is replaced by $\bigcup_{M \in \mathscr{M}} M=G$.

We are now ready for the main definition of this section.

Definition 2.6. Let $g: U(\bar{U}) \rightarrow G$ be admissible on $S \cap U(S \cap \bar{U})$. The map $g$ is called sectionally 0 -epi on $S \cap U(S \cap \bar{U})$ if and only if the equation $g(x)=h(x)$ has a solution on $S \cap U$ for any bounded map $h: E \rightarrow M$ having bounded support contained in $U(\{x \in E: h(x) \neq 0\} \subset U)$ and for any $M \in \mathscr{M}$, i.e., if $g$ is written as $g=\left(g_{M}, g_{N}\right)$, then $g_{M}$ is 0 -epi on $g_{N}^{-1}(0) \cap S \cap U\left(g_{N}^{-1}(0) \cap S \cap \bar{U}\right)$, where $N$ is such that $G=M \oplus N$.

Definition 2.7. The map $g$ will be called sectionally 0-essential on $S \cap U(S \cap \bar{U})$ if and only if for any bounded set $V$ with $g^{-1}(0) \cap S \subset V \subset \bar{V} \subset U\left(g^{-1}(0) \cap S \subset\right.$ $V \subset U$ ), any extension $\tilde{g}: \bar{V} \rightarrow G$ of the restriction $\left.g\right|_{\partial V}: \partial V \rightarrow G \backslash\{0\}$ with $g-\tilde{g}$ bounded on $\bar{V}$ and $(g-\tilde{g})(\bar{V}) \subset M$ for some $M \in \mathscr{M}$, has a zero on $S \cap V$, i.e., $g_{M}$ is 0-essential on $g_{N}^{-1}(0) \cap S \cap U\left(g_{N}^{-1}(0) \cap S \cap \bar{U}\right)$.

Clearly, from Property 2.9, if $g$ is 0 -epi on $S \cap U(S \cap \bar{U})$, then $g$ is sectionally 0-epi on $S \cap U(S \cap \bar{U})$.

Conversely, one has the following.

Property 2.10. Assume that $g$ is proper on bounded and closed (in E) subsets of $S \cap U(S \cap \bar{U})$. Then $g$ is 0-epi on $S \cap U(S \cap \bar{U})$ if and only if $g$ is sectionally 0-epi on $S \cap U(S \cap \bar{U})$.

Proof. (Only if). This implication is given by Property 2.9 (notice that the properness of $g$ is superfluous).

(If ). Suppose that $g$ is not 0 -epi on $S \cap U(S \cap \bar{U})$. Then, there exists a compact map $h: E \rightarrow G$ with bounded support contained in $U(\{x \in E: h(x) \neq 0\} \subset U)$ such that $g(x)-h(x) \neq 0$ for all $x \in S \cap U$. Since $g$ is proper on supp $h \cap S$, then $g-h$ is also proper on $\operatorname{supp} h \cap S$ and so there exists an $\varepsilon>0$ such that $\| g(x)-$ $h(x) \| \geqslant \varepsilon$ for all $x \in \operatorname{supp} h \cap S$. Now, $\overline{h(E)}$ is compact and hence there exists a finite dimensional polyhedral approximation $\tilde{h}: E \rightarrow M$ for some $M \in \mathscr{M}$, such that $\|\tilde{h}(x)-h(x)\| \leqslant \varepsilon / 4$ for all $x \in E$ (this follows easily from the fact that $\bigcup_{M \in \mathscr{M}} M$ is dense in $G$ and each vertex of the polyhedron belongs to some $M \in \mathscr{M}$ so that, by assumption (ii), $\tilde{h}(E) \subset M$ for some $M \in \mathscr{M})$. Now, the inclusion $A \equiv\{x \in E$ : $h(x)=0\} \subset\|\tilde{h}\|^{-1}([0, \varepsilon / 3))$ holds since $\|\tilde{h}\| \leqslant \varepsilon / 4$ on this set. Let $\varphi: E \rightarrow[0,1]$ be an Urysohn function with $\varphi(A)=0$ and $\varphi(B)=1$, where $B \equiv\|\tilde{h}\|^{-1}([\varepsilon / 3,+\infty))$. 
By letting $h_{M}=\varphi \tilde{h}$ we obtain that $\operatorname{supp} h_{M} \subset \operatorname{supp} h$. Moreover,

$$
\begin{aligned}
\left\|h_{M}(x)-g(x)\right\| & \geqslant\|g(x)-h(x)\|-\|h(x)-\tilde{h}(x)\|-(1-\varphi(x))\|\tilde{h}(x)\| \\
& \geqslant \varepsilon-\varepsilon / 4-\varepsilon / 3=5 \varepsilon / 12
\end{aligned}
$$

for all $x \in \operatorname{supp} h \cap S$. Furthermore, $g(x)-h_{M}(x) \neq 0$ for all $x \in(\operatorname{supp} h)^{c} \cap S$ $\cap U(\bar{U})$ from our assumption on $g$ and $h_{M}(x)=0$ on the same set. Hence, $g(x)-h_{M}(x) \neq 0$ for all $x \in S \cap U(S \cap \bar{U})$, contradicting the hypothesis. Q.E.D.

We point out that all our previous results regarding 0 -epi (0-essential) maps on $S \cap U(S \cap \bar{U})$ extend to the context of sectionally 0-epi (0-essential) maps on $S \cap U(S \cap \bar{U})$ under very small changes in the hypotheses. For the reader's convenience we shall reproduce the main results here.

Proposition 2.5. (a) The map $g$ is sectionally 0 -epi on $S \cap U(S \cap \bar{U})$ if and only if $g$ is sectionally 0-essential on $S \cap U(S \cap \bar{U})$.

(b) If $g: \bar{U} \rightarrow G$ is such that $g^{-1}(0) \cap S$ is bounded and for any closed and bounded subset $D$ of $\partial U$ one has $\operatorname{dist}\left(g_{M}\left(g_{N}^{-1}(0) \cap \bar{S} \cap D\right), 0\right)>0$ whenever $g_{N}^{-1}(0) \cap \bar{S} \cap D \neq$ $\varnothing$ and for any $M \in \mathscr{M}$, then $g$ is sectionally 0 -epi on $S \cap \bar{U}$ if and only if $g$ is sectionally 0-epi on $S \cap U$.

Properties 2.1-2.9 remain valid with the following modification for the Normalization Property 2.7. The connected component $V$ of zero in $U$ is such that $V \subset \bar{S}$ and $V \cap\left(\cup_{M \in \mathscr{M}} M\right) \subset S$. In fact, from Property 2.5 the inclusion $i$ is sectionally 0 -epi on $\bar{S} \cap U$ and is proper on it. The first conclusion follows then from Property 2.10. On the other hand, if $x_{0} \in V \cap\left(\bigcup_{M \in \mathscr{M}} M\right)$ but $x_{0} \notin S$, then let $\sigma(t)$ be a compact path from 0 to $x_{0}$ as before. By covering it with balls having centers in some $M_{i}$ and approximating it by a piecewise linear path contained in some $M$, one may proceed with the same argument as before.

Note also that Remark 2.5 has to be modified, either by defining section preserving maps (cf. [AAA,II]) or, more simply, by assuming that $S \cap U$ is closed in $U$. Indeed, since $g$ is one-to-one on $S \cap U$, it is proper and then one uses Property 2.10 .

Regarding the homotopy properties we have to pay the price of dealing with a larger class of maps. Namely, the class of admissible homotopies is smaller and, precisely, the homotopies defines on $(S \cap U) \times[0,1]$ or on $(S \cap \partial U) \times[0,1]$, as well as the extension $\tilde{h}$ of $h(\cdot, 1)$ in Corollary 2.1, and, as regards Remark 2.8, $g-\tilde{g}$, $h_{1}$ and $\tilde{h}_{1}$ must have their ranges in some element $M$ of $\mathscr{M}$ (compactness here means that bounded sets are sent into bounded sets of $M$ ).

The first advantage of our new definition concerns Proposition 2.4, since one obtains the same results under weaker assumptions. Namely, the following holds true.

Proposition 2.6. If $g$ is sectionally 0-epi on $S \cap U(S \cap \bar{U})$, then:

(a) Either $S \cap \partial V \neq \varnothing$, or $g(S \cap \bar{V})=\bigcup_{M \in M} M$ for any open and bounded set $V$ such that $g^{-1}(0) \cap S \subset V \subset \bar{V} \subset U\left(g^{-1}(0) \cap S \subset V \subset U\right)$. In particular, if $G=M$ $\oplus N$ for some $M \in \mathscr{M}$ with $\operatorname{dim} M \geqslant 1$, and if $g_{M}$ sends bounded and closed subsets of $g_{N}^{-1}(0) \cap S \cap U\left(g_{N}^{-1}(0) \cap S \cap \bar{U}\right)$ into bounded subsets of $M$, then $S \cap \partial V \neq \varnothing$. 
(b) Either, $S \cap U$ is unbounded, or $g(S \cap \bar{V})=\cup_{M \in \mathscr{M}} M$ for some $V$ as above, or $(\overline{S \cap U}) \cap \partial U \neq \varnothing$. In particular, if $S \cap \bar{U}$ is closed, then $S \cap \partial U \neq \varnothing$.

Proof. Notice first that the homotopy $t p$ is finite dimensional. It is enough to be able to choose $p$ in $\bigcup_{M \in \mathscr{M}} M$. If $g_{M}$ satisfies the above boundedness property, then one may choose $p \in M$ so large that $p \notin g_{M}\left(g_{N}^{-1}(0) \cap S \cap \bar{V}\right)$, but then $g-p$ is not sectionally 0-epi on $S \cap \bar{V}$. Q.E.D.

The hypotheses of Proposition 2.6 lead to the following two definitions which will be used, in particular, in the main result of this paper (see Theorem 3.1).

Definition 2.8. Let $X$ be a closed subset of $E$. A continuous map $g: X \rightarrow G$ will be called

(a) sectionally bounded on $X$ if and only if, for any $M \in \mathscr{M}, g_{M}\left(g_{N}^{-1}(0) \cap X\right)$ is bounded, where $N$ is such that $G=M \oplus N$ (cf. Definition 2.6),

(b) sectionally proper on $X$ if and only if for any $M \in \mathscr{M}$ and any compact set $\kappa \subset M$, the set $g^{-1}(\kappa \times\{0\})$ is compact.

REMARK 2.9. (A-proper 0-epi maps on $S \cap U$.) One could reduce further the dimensions of the Banach spaces $E$ and $G$ by using a sequence of finite dimensional subspaces $E_{n}$ and $G_{n}$ of $E$ and $G$ (not necessarily of the same dimension) and projections $Q_{n}: G \rightarrow G_{n}$. Then, if $g_{n}=\left.Q_{n} g\right|_{U \cap E_{n}=U_{n}}$ is continuous and admissible on $S \cap U_{n}$ one may ask that $g_{n}$ be 0 -epi on $S \cap U_{n}$ for infinitely many $n$ 's. Clearly, all of the previous results are valid for $g_{n}$ with very weak conditions on the $g_{n}$ itself (for example Proposition 2.3 is valid provided $g_{n}(x) \neq 0$ on $\partial U$ ). However, one may not infer any conclusion on $g$ unless further hypotheses are given. We shall assume that the following assumptions, on the (not necessarily continuous) map $g$ and projections $Q_{n}$, are satisfied.

(1) $g^{-1}(0) \cap S \subset V_{0} \subset \bar{V}_{0} \subset U$ for some open and bounded subset $V_{0}$, and $g_{n}^{-1}(0) \cap S \subset V_{0} \cap E_{n}$ for all $n$ 's.

(2) $\left\|Q_{n}\right\| \leqslant \kappa$ for all $n$ 's.

(3) If for a given $z \in G$ and for an increasing sequence of $n$ 's one has $x_{n_{\kappa}} \in S \cap$ $U_{n_{\kappa}} \cap V$, with $V$ open and bounded such that $V \subset \bar{V} \subset U$, and $g_{n_{\kappa}}\left(x_{n_{\kappa}}\right)-Q_{n_{\kappa}} z \rightarrow 0$, then $x_{n_{\kappa}}$ has a subsequence which converges to some $x \in S \cap U$, with $g(x)=z$. Under the above assumptions the following holds.

If $g_{n}$ is 0 -epi on $S \cap U_{n}$ for infinitely many $n$ 's, then the equation $g(x)=h(x)$ has a solution in $S \cap U$ for any compact map $h: E \rightarrow G$ with bounded support contained in $U$ (i.e., the only missing condition for $g$ to be 0-epi is its continuity).

Proof. Let $h: E \rightarrow G$ be as above. Then $h_{n}=\left.Q_{n} h\right|_{E_{n}}$ has bounded support contained in $E_{n}$. Hence there is $x_{n} \in S \cap U_{n}$ such that $g_{n}\left(x_{n}\right)=h_{n}\left(x_{n}\right)$ for infinitely many $n$ 's. Now, if infinitely many of the $x_{n}$ 's belong to $\operatorname{supp} h$, then they are bounded and, passing to a subsequence if necessary, we have $h\left(x_{n}\right) \rightarrow z \in G$. It follows that $Q_{n}\left(h\left(x_{n}\right)-z\right) \rightarrow 0$, because of hypothesis (2). Therefore, from (3) we get $g(x)=z=h(x)$. On the other hand, if $g_{n}\left(x_{n}\right)=0$ for infinitely many $n$ 's (i.e., $\left.x_{n} \notin \operatorname{supp} h\right)$, then $x_{n} \in V_{0} \cap E_{n}$ and, again one gets $g(x)=0$ from (1). Q.E.D.

Note that homotopies which meet (1)-(3) above are admissible in the class of A-proper 0-epi maps. 
The result we have just proved shows that the class of continuous $A$-proper 0 -epi maps is contained in that of 0 -epi maps. As a matter of fact, there exist 0 -epi maps which are not $A$-proper 0 -epi, as the following example shows. Let $E=G=l^{2}$, $S=\left\{\left(\varepsilon, \varepsilon^{2}, \varepsilon^{3}, \ldots\right):|\varepsilon|<1\right\}, G_{n}=E_{n}=$ the $n$ first components and let $g: E \rightarrow \mathbf{R}$ be defined by $g(x)=x_{1}\|x\|^{2}$. One has $\left.g\right|_{S}=\varepsilon^{3} /\left(1-\varepsilon^{2}\right), S \cap E_{n}=\{0\}$ so that $\left.g\right|_{S \cap E_{n}}$ $=\{0\}$ is not 0 -epi, $g^{-1}(0) \cap S=\{0\}$ and, if $Q_{n} z \rightarrow 0$, then $z=0=g(0)$. Hence, conditions (1)-(3) are met. But, $g$ is 0 -epi on $S$, since any map $h$ with bounded support is bounded on $S$ and $\left.g\right|_{S}$ is onto $\mathbf{R}$.

In spite of this example, the following holds. If $E=G \oplus E_{0}$ and $E_{n}=G_{n} \oplus E_{0}$ so that $\left(I-Q_{n}\right)=\left(I-P_{n}\right)$, where $P_{n}$ is the projection onto $E_{n}$, and if $\left(I-Q_{n}\right) g(x)$ $=\left(I-P_{n}\right) x+k_{n}(x)$ with $k_{n}$ compact (as it happens frequently in applications) and having the property that the homotopy $Q_{n} g(x)+\left(I-P_{n}\right) x+t k_{n}(x)$ is admissible (for large $n$ ) on $S \cap U$, then $g$ is 0-epi on $S \cap U$ if and only if $Q_{n} g(x)+$ $\left(I-P_{n}\right) x$ is 0-epi on $S \cap U$ and, from Property 2.9, $Q_{n} g$ is 0-epi on $S \cap U_{n}$ (for further results on $A$-proper 0-epi maps see Remarks 3.3 and 3.4).

Regarding the theory of $A$-proper maps, their topological degree and applications (in the case when $S=E$ ) see [BP and FMP,I].

3. Dimension. In the first part of this section we give first a result relating the concept of 0 -epi map on $S \cap \bar{U}$ with the classical notion of essential map. We proceed further with a result regarding real valued 0 -epi maps on $S \cap \bar{U}$. The last result of this part is a characterization of $A-H$ essential maps.

The second part is devoted to the study of the relation between the concept of 0 -epi maps and classical dimension theory. The dimension we shall use throughout this paper is the covering dimension, whose definition runs as follows. A normal topological space $X$ has covering dimension equal to $n$, provided that $n$ is the smallest integer with the property that whenever $\mathscr{U}$ is an open covering of $X$, there exists a refinement $\mathscr{U}^{\prime}$ of $\mathscr{U}$, which also covers $X$, and no more than $n+1$ members of $\mathscr{U}^{\prime}$ have nonempty intersection.

The third, and most important, part of this section contains the main result of this paper (Theorem 3.1). This theorem extends all of the previous results contained in [AA,I; AA,II; AMP; FMP,I; FMP,II and MP].

Theorem 3.1 shows, in particular, that if a certain map $g$ is sectionally 0 -epi on $S \cap U$ (plus some, very mild, extra assumptions), then there is a minimal closed subset of $S \cap U$ having a number of properties related, not only to its global structure, but, also, to its covering dimension at each point (local dimension).

A. 0-epi maps on $S \cap U(S \cap \bar{U})$ with finite dimensional range. The results of dimension theory that we are going to use are given in terms of maps having range in finite dimensional spheres. Using the classical notion of essentiality we shall show, in this section, that the definitions given in $\$ 2$ allow us to use these classical results.

The following result relates the classical concept of essential map with that of 0-epi map on $S \cap \bar{U}$.

Proposition 3.1. Let $U$ be bounded and $S \cap \bar{U}$ be closed. Let $g: \bar{U} \rightarrow \mathbf{R}^{n}$ be bounded on $\bar{U}$ with $g^{-1}(0) \cap S \subset U$. Then, the restriction $g: S \cap \partial U \rightarrow \mathbf{R}^{n} \backslash\{0\}$ is 
essential with respect io $S \cap \bar{U}$ (i.e., $g$ is 0 -epi on $S \cap \bar{U}$ ) if and only if $g /\|g\|$ : $S \cap \partial U \rightarrow S^{n-1}$ is not extendible over $S \cap \bar{U}$.

Proof. (Only if ). Consider the map $h:(S \cap \partial U) \times[0,1] \rightarrow \mathbf{R}^{n}$ defined by $h=$ $-\operatorname{tg}(1-\|g\|) /(1-t(1-\|g\|))$. Then the map $g-h:(S \cap \partial U) \times[0,1] \rightarrow \mathbf{R}^{n} \backslash\{0\}$ gives an admissible homotopy (notice that $h$ is compact since $\|h\| \leqslant\|1-\| g \| \mid$ ). By Corollary 2.1(b), if $g$ is 0 -epi on $S \cap \bar{U}$, so is $g-\tilde{h}$, where $\tilde{h}$ is any extension (to $\bar{U}$ ) of $h(\cdot, 1)$. We have that $g-\tilde{h}=g /\|g\|$ on $S \cap \partial U$. However, if $g /\|g\|$ has an extension $\hat{g}$ : $S \cap \bar{U} \rightarrow S^{n-1}$, then, taking $g-\tilde{h}$ to be $\hat{g}$ on $S \cap \bar{U}$ and any continuous extension of $\hat{g}$ outside $S \cap \bar{U}, g-\tilde{h}$ would be nonvanishing on $S \cap \bar{U}$, contradicting Property 2.1 .

(If). Assume that $g: S \cap \partial U \rightarrow \mathbf{R}^{n} \backslash\{0\}$ has a bounded continuous extension $\hat{g}$ : $S \cap \bar{U} \rightarrow \mathbf{R}^{n} \backslash\{0\}$. Then $\hat{g} /\|\hat{g}\|$ is an extension of $g /\|g\|$. Q.E.D.

Corollary 3.1. If $U, S$ are as above and if $g: \bar{U} \rightarrow \mathbf{R}$ is 0-epi on $S \cap \bar{U}$, then $S \cap \partial U$ has at least two components on which $g$ changes sign.

Proof. If $g$ does not change sign on $S \cap \partial U$, then, clearly, $g /\|g\|$ has a constant $( \pm 1)$ extension to $S \cap \bar{U}$. This contradicts Proposition 3.1. Q.E.D.

The following definition has been extensively used in the literature regarding classical dimension theory (see [GT] for a recent survey on this subject).

Definition 3.1. Let $X$ be a topological space and let $I^{n}$ denote the unit cube in $\mathbf{R}^{n}$. A map $g: X \rightarrow I^{n}$ is called $A$-H-inessential if and only if there exists a map $g_{1}$ : $X \rightarrow I^{n}$ such that $g_{1}(x)=g(x)$ for all $x \in g^{-1}\left(\partial I^{n}\right)$ and $g_{1}(X) \neq I^{n}$ (i.e., $g_{1}$ is not onto) (see e.g. [GT]).

Proposition 3.2. The map $g: X \rightarrow I^{n}$ is $A$-H-inessential if and only if $g: g^{-1}\left(\partial I^{n}\right)$ $\rightarrow \partial I^{n}$ is continuously extendible over $X$.

Proof. (If ). Assume that $g: g^{-1}\left(\partial I^{n}\right) \rightarrow \partial I^{n}$ is continuously extendible over $X$. Then, clearly, $g$ is $A$ - $H$-inessential since one can take $g_{1}=g$.

(Only if). Assume that there exists $g_{1}: X \rightarrow I^{n}$ such that it coincides with $g$ on $g^{-1}\left(\partial I^{n}\right)$ and that for some $\lambda_{0} \in I^{n}$ we have that $g_{1}(x) \neq \lambda_{0}$ for all $x \in X$. Put $\tilde{g}(x)=t\left(g_{1}(x)\right)\left(g(x)-\lambda_{0}\right)+\lambda_{0}$, where $t\left(g_{1}(x)\right)$ is the unique positive solution of $\left\|\lambda t+(1-t) \lambda_{0}\right\|=1$ for $\lambda \in I^{n}$. Clearly, $\tilde{g}$ is a continuous extension of $g$ over $X$. Q.E.D.

B. Preliminary results on dimension. Note that, in most of the results given up to now, we have only used the normality of the space $E$ (the metric of $E$ has been used, because of our definitions, when dealing with bounded sets). However, in what follows, in order to avoid complications in the definitions, the metric of $E$ will be used in a crucial way.

For metric spaces the three most widely used concepts of dimension of a set $X$, namely, the small inductive dimension of $X$, ind $X$, the large inductive dimension of $X$, Ind $X$ and the covering dimension of $X, \operatorname{dim} X$, are related by

$$
\text { ind } X \leqslant \text { Ind } X=\operatorname{dim} X \text {, }
$$

where the first inequality is replaced by equality if $X$ is separable [P, pp. 156 and 181]. 
As a warning for the reader who is unfamiliar with dimension theory we have the following: If $A$ is a subset of a metric space $X$, then $\operatorname{dim} A \leqslant \operatorname{dim} X$ provided that $A$ is closed, while ind $A \leqslant$ ind $X$ for any $A \subset X[\mathbf{P}$, pp. 152 and 158].

In the remainder of this paper we shall use the covering dimension.

The following is a result relating 0 -epi maps on $S \cap \bar{U}$ with dimension theory.

Proposition 3.3. Let $U$ be bounded, $S \cap \bar{U}$ be closed and $g: \bar{U} \rightarrow \mathbf{R}^{n}$ be bounded on $\bar{U}$. Assume that $g$ is 0 -epi on $S \cap \bar{U}$. Then $\operatorname{dim}(S \cap \bar{U}) \geqslant n$ and $\operatorname{dim}(S \cap \partial U) \geqslant$ $n-1$.

Proof. The first inequality follows from [P, p. 127] and Propositions 3.1 and 3.2.

The second inequality follows from the fact that the set

$$
A=\left\{x \in S \cap \partial U: g(x) /\|g(x)\| \neq p \in S^{n-1}\right\}
$$

can be written as $A=\bigcup_{j=1}^{+\infty} \bar{U}_{j}$, with $U_{j}=\left\{x \in S \cap \partial U:\left\|f_{0}(x)-f_{1}(x)\right\|>j^{-1}\right\}$, where $f_{0}=g /\|g\|$ and $f_{1}=p$. By the Countable Sum Theorem [P, p. 125] $\operatorname{dim} A \leqslant$ $\operatorname{dim}(S \cap \partial U)$, so that if $\operatorname{dim}(S \cap \partial U)<n-1$, then $g /\|g\|$ and the constant map $p$ are uniformly homotopic [P, p. 333]. This implies that $g /\|g\|$ has a nonzero extension to $S \cap \bar{U}$. This contradicts Proposition 3.1 since $g$ on $S \cap \partial U$ cannot have a nonzero extension to $S \cap \bar{U}$. Q.E.D.

In the case when the range of the map $g$ is infinite dimensional we have the following.

Proposition 3.4. Let $U$ be bounded, $g: \bar{U} \rightarrow G$ be sectionally 0-epi and sectionally bounded on $S \cap \bar{U}$. If $\operatorname{dim} G=+\infty$, then $\operatorname{dim}(S \cap \bar{U})=\operatorname{dim}(S \cap \partial U)=+\infty$.

Proof. Assume that $\operatorname{dim}(S \cap \bar{U}) \leqslant n<+\infty$. Let $M \in \mathscr{M}$ be a $2 n$-dimensional subspace of $G$ and let $N$ be a topological complement of $M$. Write $g=\left(g_{M}, g_{N}\right)$, where $g_{M}(\bar{U}) \subset M$. Then, by Property 2.9, the map $g_{M}$ is 0 -epi on $g_{N}^{-1}(0) \cap S \cap \bar{U}$. Therefore, by Proposition 3.3

$$
\operatorname{dim}\left(g_{N}^{-1}(0) \cap S \cap \bar{U}\right) \geqslant 2 n,
$$

contradicting $\operatorname{dim}(S \cap \bar{U}) \leqslant n$.

Assuming $\operatorname{dim}(S \cap \partial U) \leqslant n-1$, the same argument yields

$$
\operatorname{dim}\left(g_{N}^{-1}(0) \cap S \cap \partial U\right) \geqslant 2 n-1 \text {. Q.E.D. }
$$

REMARK 3.1. By Propositions 3.3 and 3.4 we have that if $g$ is sectionally 0 -epi on $S \cap U(S \cap \bar{U})$ and sectionally bounded on bounded and closed (in $E$ ) subsets of $S \cap U(S \cap \bar{U})$, then $\operatorname{dim}(S \cap \bar{V}) \geqslant \operatorname{dim} G$ and $\operatorname{dim}(S \cap \partial V) \geqslant \operatorname{dim} G-1$ for any open bounded set $V$ such that $g^{-1}(0) \cap S \subset V \subset \bar{V} \subset U\left(g^{-1}(0) \cap S \subset V \subset U\right)$. This implies, in particular, that $\operatorname{dim}(S \cap U) \geqslant \operatorname{dim} G$ (notice that $S \cap \bar{V}$ is closed in $S \cap U)$.

In the case when $g$ is sectionally 0 -epi on $S \cap \bar{U}$ and $S \cap \bar{U}$ is bounded, then $\operatorname{dim}(S \cap \partial U) \geqslant \operatorname{dim} G-1$ (take a sufficiently large ball $B$ such that $S \cap \bar{U} \subset B$ and let $V=U \cap B$; then, clearly, $S \cap \partial V \subset S \cap \partial U$ ).

C. Main results on dimension. The following technical lemma will be used in the principal result of this paper. 
Lemma 3.1. Let $A=A_{1} \cup A_{2}$, where $A_{1}, A_{2}$ are bounded and closed subsets of $A$. Let $g, g_{1}, g_{2}: A \rightarrow G$ be continuous maps which are sectionally bounded on $A$ and satisfy the following assumptions.

(i) $g_{i}-g$ is compact, $i=1,2$.

(ii) $g_{i}: A_{i} \rightarrow G \backslash\{0\}, i=1,2$.

(iii) There exist a closed subset $B_{i} \subset A_{i}$ such that $g_{i}=g$ on $B_{i}, i=1,2$.

(iv) $\operatorname{dim}\left\{x \in A_{1} \cap A_{2}: g_{1}(x) /\left\|g_{1}(x)\right\| \neq g_{2}(x) /\left\|g_{2}(x)\right\|\right\}<\operatorname{dim} G-1$ if $g_{i}-g$ are finite dimensional maps (i.e., with range in some $M \in \mathscr{M}$ ),

$$
\operatorname{dim}\left(A_{1} \cap A_{2} \backslash\left(B_{1} \cap B_{2}\right)\right)<+\infty
$$

if $\operatorname{dim} G=+\infty$, in which case $g$ is assumed to be proper.

Then, the restrictions $\left.g\right|_{B_{1} \cup B_{2}},\left.g_{i}\right|_{A_{i}}, i=1,2$, extend to $\tilde{g}, \tilde{g}_{i}: A \rightarrow G \backslash\{0\}, i=1,2$, with $g-\tilde{g}$ and $g_{i}-\tilde{g}_{i}, i=1,2$, compact on $A$. (If $g_{i}-g$ are finite dimensional, then $g-\tilde{g}, g_{i}-\tilde{g}_{i}$ have range in some $M \in \mathscr{M}$.)

Proof. (a) $\left(\operatorname{dim} G=n\right.$.) From $\left[\mathbf{P}\right.$, p. 334] each $g_{i} /\left\|g_{i}\right\|$ has an extension to $A$ so that $g /\left.\|g\|\right|_{B_{1} \cup B_{2}}$ extends to $A$. The result now follows from Proposition 3.1.

(b) $\left(\operatorname{dim} G=+\infty\right.$ and $\operatorname{dim}\left\{x \in A_{1} \cap A_{2}: g_{1}(x) /\left\|g_{1}(x)\right\| \neq g_{2}(x) /\left\|g_{2}(x)\right\|\right\}<n$ - 1 and $g_{1}-g$ are finite dimensional maps.) Decompose the space $G$ as a direct sum $M \oplus N$, where $M$ is finite dimensional, $\operatorname{dim} M \geqslant 2 n$, and $g_{i}-g: A \rightarrow M \in \mathscr{M}$. Write $g=\left(g_{M}, g_{N}\right)$; then

$$
g_{i}=g_{i}-g+g=\left(g_{i}-g+g_{M}, g_{N}\right)
$$

so that the restriction $\left.g_{M}\right|_{g_{N}^{-1}(0) \cap B_{i}}$ has an extension to $g_{N}^{-1}(0) \cap A_{i}, i=1,2$, via $g_{M}+\left(g_{i}-g\right)=\left.g_{i}\right|_{g_{N}^{-1}(0) \cap A_{i}}$. However, the set

$\left\{x \in g_{N}^{-1}(0) \cap A_{1} \cap A_{2}: \frac{g_{M}(x)+g_{i}(x)-g(x)}{\left\|g_{M}(x)+g_{i}(x)-g(x)\right\|} \neq \frac{g_{M}(x)+g_{2}(x)-g(x)}{\left\|g_{M}(x)+g_{2}(x)-g(x)\right\|}\right\}$

is a closed subset of the set $\left\{x \in A_{1} \cap A_{2}: g_{1}(x) /\left\|g_{1}(x)\right\| \neq g_{2}(x) /\left\|g_{2}(x)\right\|\right\}$ and hence it has dimension less than $n-1$. Therefore, from the first part of the proof, it follows that $\left.\left(g_{M}+g_{i}-g\right)\right|_{g_{N}^{-1}(0) \cap A_{i}}, i=1,2$, and $\left.g_{M}\right|_{g_{N}^{-1}(0) \cap\left(B_{1} \cup B_{2}\right)}$ have extensions to $g_{N}^{-1}(0) \cap A$ and so the maps $\left(g_{M}+g_{i}-g, g_{N}\right)=g_{i}, i=1,2$, and $\left(g_{M}, g_{N}\right)=g$ will have finite dimensional extensions.

(c) $\left(\operatorname{dim} G=+\infty, \operatorname{dim}\left(A_{1} \cap A_{2} \backslash\left(B_{1} \cap B_{2}\right)\right)<n-1\right.$ and $g$ proper.) Since $g_{i}=$ $g_{i}+g-g$ is proper (this follows from the fact that $g$ is proper and $g_{i}-g$ is compact), $i=1,2$, there exists $\varepsilon>0$ such that $\left\|g_{i}(x)\right\| \geqslant \varepsilon$ for all $x \in A_{i}, i=1,2$. Now, $g_{i}-g$ can be approximated within $\varepsilon / 4$ on $A$ by a finite dimensional map $h_{i}$. Furthermore, as in the proof of Property 2.10, one may replace $h_{i}$ by $\tilde{h}_{i}=\varphi_{i} h_{i}$ with $\varphi_{i}\left(B_{i}\right)=0$ and $\left\|g(x)+\tilde{h}_{i}(x)\right\| \geqslant 5 \varepsilon / 12$ for all $x \in A_{i}$ so that the map $g+\tilde{h}_{i}$ extends $g$ on $B_{i}$ to $A_{i}$ with the finite dimensional map $\tilde{h}_{i}$. Now, $\operatorname{since} \operatorname{dim}\left(A_{1} \cap A_{2}\right)$ $<n-1$, then the set

$$
\left\{x \in A_{1} \cap A_{2}: \frac{g(x)+\tilde{h}_{1}(x)}{\left\|g(x)+\tilde{h}_{1}(x)\right\|} \neq \frac{g(x)+\tilde{h}_{2}(x)}{\left\|g(x)+\tilde{h}_{2}(x)\right\|}\right\}
$$


also has dimension less than $n-1$, being a countable union of closed sets (cf. the proof of Proposition 3.3). Then, $\left(g_{M}+\tilde{h}_{i}, g_{N}\right)=g+\tilde{h}_{i}$ and $\left(g_{M}, g_{N}\right)=g$ have finite dimensional extensions to $A$ as follows from (b).

Finally, since $g_{i}$ and $g+\tilde{h}_{i}$ are $7 \varepsilon / 12$-close on $A_{i}$ and compactly homotopic (via the homotopy $\left.g_{i}-t\left(g_{i}-g-\tilde{h}_{i}\right)\right)$, then $g_{i}$ has a compact extension to $A$ (see Remark 2.8). Q.E.D.

In Lemma 3.1 the properness assumption on $g$ seems to be unnecessary. It was needed for the approximation process since the dimension results used here are for mappings with finite dimensional range.

The following result can be viewed as a further interesting property of 0 -epi maps on $S \cap \bar{U}$.

Proposition 3.5. Let g: $\bar{U} \rightarrow G$ be sectionally 0-epi on $S \cap \bar{U}$ and sectionally bounded on bounded and closed subsets of $S \cap \bar{U}$. Assume that the following property holds.

$$
g^{-1}([0, p]) \cap S \text { is bounded for any } p \in \bigcup_{M \in \mathscr{M}} M .
$$

Then $\operatorname{dim}(S \cap \partial U)<\operatorname{dim} G-1$ implies $g(S \cap \bar{U}) \supset \cup_{M \in \mathscr{M}} M$. (Note that if the family $\mathscr{M}$ is taken to be that of all finite dimensional subspaces of $G$, then $G=\cup_{M \in \mathscr{M}} M$.)

Proof. From Remark 3.1, $S \cap \bar{U}$ cannot be bounded. Assume now that $g(x) \neq p$ for some $p \in \cup_{M \in \mathscr{M}} M$ and all $x \in S \cap \bar{U}$. Since $g^{-1}([0, p]) \cap S$ is bounded, take $R>0$ so large that this set is contained in a ball $B_{R}$ centered at the origin with radius $R$. Let $\varphi: \mathbf{R}^{+} \rightarrow \mathbf{R}^{+}$be an increasing function such that $\varphi(r)=0$ if $0 \leqslant r \leqslant R$, and $\varphi(r)=1$ if $r \geqslant 2 R$. Now, on $S \cap \partial\left(\bar{B}_{2 R} \cap \bar{U}\right)$ consider the admissible homotopy

$$
g(x, t)=g(x)-t \varphi(\|x\|) p .
$$

From Corollary 2.1(b) the map $g-\varphi p$ is sectionally 0-epi on $S \cap \bar{B}_{2 R} \cap \bar{U}$. Apply Lemma 3.1 with $A=S \cap \bar{B}_{2 R} \cap \bar{U}, A_{1}=S \cap \partial\left(\bar{B}_{2 R} \cap \bar{U}\right), A_{2}=A, B_{1}=S \cap \bar{B}_{R}$ $\cap \partial U, B_{2}=\varnothing, g_{1}=g-\varphi p$ and $g_{2}=g-p$ (observe that $g_{1}$ and $g_{2}$ differ on a subset of $S \cap \partial U$ which, by assumption, has $\operatorname{dimension}$ less than $\operatorname{dim} G-1)$. We obtain that $g_{1}$ has a nonvanishing extension to $A$, contradicting the fact that $g_{1}$ is sectionally 0-epi on $A$. Q.E.D.

REMARK 3.2. If $g$ is either sectionally proper on $S \cap \bar{U}$, or $\|g(x)\| \rightarrow+\infty$ as $\|x\| \rightarrow+\infty, x \in S \cap \bar{U}$, then $g$ satisfies property (*) of Proposition 3.5.

The following theorem is the main result of this paper.

THEOREM 3.1. Let $S$ be closed in $U$ and let $g: U \rightarrow G$ be sectionally 0-epi on $S \cap U$. Assume that $g$ is sectionally proper and sectionally bounded on bounded and closed (in $E$ ) subsets of $S \cap U$. Then, there exists a minimal closed (in $U$ ) subset $\Sigma \subset S \cap U$ such that:

(a) $g$ is sectionally 0 -epi on $\Sigma \cap U=\Sigma$. This implies, in particular, that $g^{-1}(0) \cap \Sigma$ $\neq \varnothing ; \Sigma$ is either unbounded, or $\bar{\Sigma} \cap \partial U \neq \varnothing ; \operatorname{dim} \Sigma \geqslant \operatorname{dim}(\Sigma \cap \bar{V}) \geqslant \operatorname{dim} G$ and $\operatorname{dim}(\Sigma \cap \partial V) \geqslant \operatorname{dim} G-1$ for any open and bounded set $V$ such that $g^{-1}(0) \cap S \subset V$ $\subset \bar{V} \subset U$. 
(b) If $\Sigma=\Sigma_{1} \cup \Sigma_{2}$, with $\Sigma_{1}, \Sigma_{2}$ closed (in $\Sigma$ ) and proper subsets of $\Sigma$, then $\operatorname{dim}\left(\Sigma_{1} \cap \Sigma_{2}\right) \geqslant \operatorname{dim} G-1$. This implies, in particular, that $\Sigma$ is connected and has dimension at each point at least $\operatorname{dim} G$.

(c) $\Sigma$ is minimal for any map $g_{1}$ homotopic to $g$ via a finite dimensional homotopy and, if $g$ and $g_{1}$ are proper on bounded and closed subsets of $S \cap U$, then $\Sigma$ is minimal for any map $g_{1}$ homotopic to $g$.

(d) If $\bar{\Sigma}=\Sigma_{1} \cup \Sigma_{2}$, where $\Sigma_{1}, \Sigma_{2}$ are closed and proper subsets of $\bar{\Sigma}$, then $\operatorname{dim}\left(\Sigma_{1} \cap \Sigma_{2} \cap U\right) \geqslant \operatorname{dim} G-1$, and there is no proper and closed subset $C \subset \bar{\Sigma}$ such that $g$ is sectionally 0 -epi on $C \cap U$. In particular, $\bar{\Sigma}$ is connected and has dimension at each point at least $\operatorname{dim} G$. In fact, $\operatorname{dim}(\bar{W} \cap U) \geqslant \operatorname{dim} G$ for any open neighborhood $W$ of $p$ in $\bar{\Sigma}$.

(e) If $g$ is defined on $\bar{U}$ with $g(x) \neq 0$ on $\bar{\Sigma} \cap \partial U$ and if $g$ is sectionally proper and sectionally bounded on bounded and closed subsets of $\bar{\Sigma} \cap \bar{U}$, then $g$ is sectionally 0-epi on $\bar{\Sigma} \cap \bar{U}=\bar{\Sigma}$ and there is no proper and closed subset of $\bar{\Sigma} \cap \bar{U}$ on which $g$ is sectionally 0-epi, i.e., $\bar{\Sigma}$ is minimal. Furthermore, $\operatorname{dim}(\bar{\Sigma} \cap \partial V) \geqslant \operatorname{dim} G-1$ for any open and bounded subset $V$ with $g^{-1}(0) \cap \Sigma \subset V \subset U$ and one can take $V=U$ if $\bar{\Sigma} \cap \bar{U}$ is bounded. Finally, if $g$ satisfies property $(*)$ of Proposition 3.5 and $\operatorname{dim}(\bar{\Sigma} \cap \partial U)<\operatorname{dim} G-1$, then $g(\bar{\Sigma}) \supset \bigcup_{M \in \mathscr{M}} M$.

(Sectionally bounded and sectionally proper maps are given in Definition 2.8.)

Proof. (a) Since $g$ is sectionally proper on bounded and closed (in $E$ ) subsets of $S \cap U$ and $g^{-1}(0) \cap S$ is such a set, then $g^{-1}(0) \cap S$ is compact. Now, let $\mathscr{C}$ be the family

$$
\mathscr{C}=\{C \subset S, C \text { closed in } S \cap U: g \text { is sectionally 0-epi on } C \cap U\} .
$$

The family $\mathscr{C}$ is not empty since $S \cap U \in \mathscr{C}$. Define an order in $\mathscr{C}$ by inclusion of sets and let $\mathscr{C}^{\prime}$ be a chain in $\mathscr{C}$. Consider $\Sigma=\bigcap_{C \in \mathscr{C}^{\prime}} C$. Since $g^{-1}(0) \cap C$ is a descending family of compact sets (notice that $g^{-1}(0) \cap C \neq \varnothing$ by Property 2.1), then $g^{-1}(0) \cap \Sigma$ is nonempty and compact.

Let $h: E \rightarrow M \in \mathscr{M}$ be compact with bounded support contained in $U$ and let $V_{0}$ be an open and bounded set such that $g^{-1}(0) \cap S \subset V_{0} \subset \bar{V}_{0} \subset U$. Set $V_{1}=V_{0} \cup$ $\{x \in E: h(x) \neq 0\}$ which is bounded, open and $\bar{V}_{1} \subset U$. Writing $g=\left(g_{M}, g_{N}\right)$, since $g_{M}$ is proper on $g_{N}^{-1}(0) \cap S \cap \bar{V}_{1}$ and $h$ is compact, we have that $g_{M}-h$ is proper on $g_{N}^{-1}(0) \cap S \cap \bar{V}_{1}$. Therefore, $(g-h)^{-1}(0) \cap S \cap \bar{V}_{1}$ is a compact set and $(g-h)^{-1}(0) \cap C \cap \bar{V}_{1}$ is a descending family of compact sets. These sets are nonempty since $g$ is sectionally 0 -epi on $C \cap U, g(x)=h(x)$ has a solution in $C \cap U$, but $h(x)=0$ and $g(x) \neq 0$ for all $x \in S \cap V_{1}^{c}$; thus the solution is in $C \cap \bar{V}_{1}$. Hence, $(g-h)^{-1}(0) \cap \Sigma \cap \bar{V}_{1} \neq \varnothing$, i.e., $g$ is sectionally 0-epi on $\Sigma \cap U$.

By Zorn's Lemma, $\mathscr{C}$ has a minimal element, also denoted $\Sigma$. Since $g$ is sectionally 0 -epi on $\Sigma \cap U$ then, by minimality, $\Sigma \subset S \cap U$. In particular, since $g$ is sectionally bounded on bounded and closed (in $E$ ) subsets of $\Sigma$, we get that the remaining part of (a) follows from Property 2.1, Proposition 2.6 and Remark 3.1.

(b) Assume now that $\Sigma=\Sigma_{1} \cup \Sigma_{2}$, where $\Sigma_{1}, \Sigma_{2}$ are closed proper subsets of $\Sigma$ such that $\operatorname{dim}\left(\Sigma_{1} \cap \Sigma_{2}\right)<\operatorname{dim} G-1$. Since $\Sigma_{1}, \Sigma_{2}$ are closed proper subsets of $\Sigma$, it follows that $g$ is not sectionally 0 -epi on $\Sigma_{i} \cap U, i=1,2$. Therefore, there exist open 
and bounded sets $V_{i}$ with $g^{-1}(0) \cap \Sigma_{i} \subset V_{i} \subset \bar{V}_{i} \subset U$ such that $\left.g\right|_{\Sigma_{i} \cap \partial V_{i}}: \Sigma_{i} \cap \partial V_{i} \rightarrow$ $G \backslash\{0\}$ extends to $\tilde{g}_{i}: \Sigma_{i} \cap \bar{V}_{i} \rightarrow G \backslash\{0\}$, with $\left(\tilde{g}_{i}-g\right)\left(\bar{V}_{i}\right)$ bounded in some $M \in \mathscr{M}, i=1,2$. We shall apply Lemma 3.1. To this aim let $V=V_{1} \cup V_{2}, A=\Sigma$ $\cap \bar{V}, A_{i}=\left(\Sigma_{i} \cap \bar{V}\right) \cup(\Sigma \cap \partial V)$ and $B_{i}=\left(\Sigma_{i} \cap\left(\bar{V} \backslash V_{i}\right)\right) \cup(\Sigma \cap \partial V), \quad i=1,2$. Take a compact finite dimensional extension $h_{j}$ to $A_{j}$ of the map defined as $\tilde{g}_{i}-g$ on $\Sigma_{i} \cap \bar{V}_{i}$ and zero on $B_{i}, j \neq i, i, j=1,2$. Set

$$
g_{i}= \begin{cases}g & \text { on } B_{i}, \\ \tilde{g}_{i} & \text { on } \Sigma_{i} \cap \bar{V}_{i}, \\ g+h_{j} & \text { on } A_{j}, j \neq i,\end{cases}
$$

$i, j=1,2$. Since $g \neq 0$ on $B_{i}$, then $g_{i} \neq 0$ on $A_{i}$. Moreover, $g_{1}$ and $g_{2}$ restricted to $A_{1} \cap A_{2}$ differ on a subset of $\Sigma_{1} \cap \Sigma_{2} \cap \bar{V}$ so that assumption (iv) of Lemma 3.1 is also satisfied. Therefore $\left.g\right|_{B_{1} \cup B_{2}}$ has a nonvanishing extension $\tilde{g}$ to $A$ with $(g-\tilde{g})(A)$ bounded in $M$. In particular, $\left.g\right|_{\Sigma \cap \partial V}$ has an extension to $\Sigma \cap \bar{V}$, contradicting the fact that $g$ is sectionally 0 -epi on $\Sigma$.

It remains to show that $\Sigma$ is connected and has dimension at each point at least $\operatorname{dim} G$.

If $\Sigma$ is not connected, let $\Sigma_{1}, \Sigma_{2}$ be a partition of $\Sigma$. Then $\Sigma_{1} \cap \Sigma_{2}=\varnothing$ and so $\Sigma_{1}, \Sigma_{2}$ cannot be proper subsets of $\Sigma$, i.e., $\Sigma$ must be connected.

Finally, let $p \in \Sigma$ and recall that $\operatorname{dim}_{p} \Sigma \leqslant m$ if for each neighborhood $V$ of $p$ in $\Sigma$ there is an open set $W$ such that $p \in W \subset V$ and $\operatorname{dim} \bar{W} \leqslant m$ [P, p. 197]. Now for any open neighborhood $W$ of $p$, let $\Sigma_{1}=\Sigma \backslash W$ and $\Sigma_{2}=\bar{W}$. Then $\operatorname{dim}\left(\Sigma_{1} \cap \Sigma_{2}\right)$ $=\operatorname{dim} \partial W \geqslant \operatorname{dim} G-1$. If $\operatorname{dim} G=n$, then $\operatorname{dim} \bar{W} \geqslant n[\mathbf{P}$, p. 160] and, if $\operatorname{dim} G=$ $+\infty$, then $\operatorname{dim} \bar{W} \geqslant \operatorname{dim} \partial W=+\infty$ and hence $\operatorname{dim}_{p} \Sigma \geqslant \operatorname{dim} G$.

(c) Let $g(\cdot)-h(\cdot, t)$ be the homotopy joining $g$ with $g_{1}=g-h_{1}$. Then, by Corollary 2.1(a), $g_{1}$ is sectionally 0 -epi on $\Sigma \cap U$. Since $h_{1}$ is compact on $S \cap U$, then $g_{1}$ is sectionally proper and sectionally bounded on closed and bounded subsets of $\Sigma \cap U$. From (a) there is a minimal set $\Sigma_{1} \subset \Sigma$ on which $g-h_{1}$ is sectionally 0 -epi. Since, obviously, the homotopy is reversible, then $g$ is sectionally 0 -epi on $\Sigma_{1}$ and hence $\Sigma_{1}=\Sigma$. If $g$ and $g_{1}$ are proper on bounded and closed subsets of $S \cap U$, then use Property 2.10 to get the same result.

(d) Note first that since $S$ is closed in $U$, then $S \cap U=\bar{S} \cap U$, so that from Properties 2.5 and 2.6 the map $g$ is sectionally 0-epi on $S \cap U$ if and only if $g$ is sectionally 0 -epi on $\bar{S} \cap U$. This implies the minimality of $\bar{\Sigma}$. If $\bar{\Sigma}=\Sigma_{1} \cup \Sigma_{2}$, where $\Sigma_{1}, \Sigma_{2}$ are closed and proper subsets of $\bar{\Sigma}$, then $\Sigma=\left(\Sigma_{1} \cap U\right) \cup\left(\Sigma_{2} \cap U\right)$ with $\Sigma_{1} \cap U$ and $\Sigma_{2} \cap U$ closed in $\Sigma$. If $\Sigma_{1} \cap U=\Sigma$, then $\overline{\Sigma_{1} \cap U}=\bar{\Sigma} \subset \Sigma_{1} \cap \bar{U}=\Sigma_{1}$, so that $\Sigma_{1} \cap U$ and $\Sigma_{2} \cap U$ are proper subsets of $\Sigma$. If $p$ is a point of $\bar{\Sigma} \cap \partial U$ and $W$ is an open neighborhood of $p$ in $\bar{\Sigma}$, then $(\partial W) \cap U$ is closed in $\bar{W} \cap U$ and $\operatorname{dim}(\bar{W} \cap U) \geqslant \operatorname{dim} G$.

(e) From the hypothesis we have that $g^{-1}(0) \cap \bar{\Sigma}=g^{-1}(0) \cap \Sigma$ which is a compact subset of $U$. If $D$ is any closed and bounded subset of $\partial U$ such that $g_{N}^{-1}(0) \cap \bar{\Sigma} \cap D \neq \varnothing$, then, since $g$ is sectionally proper on $\bar{\Sigma} \cap D$, the hypotheses of Proposition 2.5 are met and $g$ is sectionally 0 -epi on $\bar{\Sigma} \cap \bar{U}$. Similarly, $g$ is sectionally 
0-epi on $C \cap \bar{U}$ if and only if $g$ is sectionally 0-epi on ( $C \cap U) \cap U$ for any closed subset $C$ of $\bar{\Sigma}$, using the above argument and Properties 2.5 and 2.6. The minimality of $\bar{\Sigma}$ follows as in (d) and the remainder of the proof is a consequence of Remark 3.1 and Proposition 3.5. Q.E.D.

We wish to point out that the idea of the proof of (a) and (b) of Theorem 3.1 is close to that of the existence of a Cantor space in a compact Hausdorff space [P, p. 335].

As mentioned earlier Theorem 3.1 contains, as particular cases, the dimension result obtained in [AA,I; AA,II; AMP; FMP,I; FMP,II and MP] (see §4).

REMARK 3.3. If $g$ is $A$-proper 0-epi on $S \cap U$ (see Remark 2.9) and sectionally proper on bounded and closed subsets of $S \cap U$ ( $g$ not necessarily continuous), it is not difficult to see that one gets a minimal set $\Sigma$. Moreover, $\Sigma \cap U_{n}$ has, in its turn, a minimal set $\Sigma_{n}$ on which $g_{n}$ is 0 -epi for infinitely many $n$ 's. Furthermore, if $\Sigma=\Sigma_{1} \cup \Sigma_{2}\left(\Sigma_{1}, \Sigma_{2}\right.$ proper and closed subsets of $\left.\Sigma\right)$, then $\operatorname{dim}\left(\Sigma_{1} \cap \Sigma_{2} \cap E_{n}\right) \geqslant$ $\operatorname{dim} G_{n}-1$ for infinitely many $n$ 's. Therefore, all the remaining conclusions of Theorem 3.1 are valid. In fact, stronger results are obtained, since one gets dimensional results on $E_{n}$ for infinitely many $n$ 's.

REMARK 3.4. Since $g$ is sectionally 0 -epi on $\Sigma \cap U$, then $g_{M}$ is 0 -epi on $g_{N}^{-1}(0) \cap \Sigma$ $\cap U$ and, as such, $g_{N}^{-1}(0) \cap \Sigma$ has a minimal set $\Sigma_{M}$ on which $g_{M}$ is 0 -epi. In general, $\Sigma_{M}$ is not the whole of $g_{N}^{-1}(0) \cap \Sigma$. As an example of this fact, take $S=E=G=\mathbf{R}^{2}$, $g(x, y)=\left(x^{3}-x, y\right)$. Then, the homotopy $\left(x\left(x^{2}-(1-t)\right), y\right)$ is admissible and $\Sigma$ is minimal (notice that, from Property 2.7, we have $\Sigma=\mathbf{R}^{2}$ ). However, $g_{1}^{-1}(0) \cap \Sigma$ $=\{x=0, x= \pm 1\}$ which is not connected.

4. Applications. In this final section we shall return to our original motivation; that is, the study of global branches of solutions to multiparameter nonlinear equations.

We shall show, in particular, how our main result on dimension can be applied to continuation, global implicit function and global bifurcation theorems.

We will recover and improve all of the previous results [AA,I; AA,II; AMP; FMP,I; FMP,II and MP], where topological degree arguments have been used. Furthermore, we shall give similar results in the case when degree theory cannot be applied as, for example, in continuation problems with Fredholm operators of positive index and bifurcation problems having bifurcation set of codimension larger than one.

In this section the set $S$ considered in Theorem 3.1 will be a subset of the zeros of a map $f: E \times \Lambda \rightarrow F$, where $E, \Lambda$ and $F$ are Banach spaces. The choice of the map $g$, that will turn out to be 0 -epi on $S \cap U$, will depend on each particular application. In order to prove that $g$ is 0 -epi on $S \cap U$, the only topological hypothesis will be that the pair $(f, g)$ has a nontrivial stable homotopy class on a suitable small sphere. We shall also give specific conditions yielding the nontriviality of this homotopy class. From this local hypothesis we shall prove that $(f, g)$ is 0 -epi on some appropriate open set $U$ and thus, from Property 2.9, obtain that $g$ is 0 -epi on $S \cap U$.

In all of the previous papers mentioned above the local topological invariant is the topological degree of the pair $(f, g)$. This implies, in general, that $f$ must be of a certain special form. 
The first result of this section enables us to show that, under appropriate control on the zeros of the pair $(f, g),(f, g)$ is 0-epi on a small ball if and only if $(f, g)$ is 0 -epi on any large open set $U$. This technical result is a good substitute for the excision property existing in classical topological degree theory.

In this section $\alpha(A)$ will stand for the Kuratowski measure of noncompactness of $A$ (see e.g. [N]).

A. Scaling property and consequences. The following result can be regarded as a further and useful property of 0-epi maps.

Proposition 4.1 (ScAling Property). Let $E=E_{1} \times E_{2}$ and $G=G_{1} \times G_{2}$, with the maximum norm, i.e., $\|\cdot\|=\max \left\{\|\cdot\|_{1},\|\cdot\|_{2}\right\}$, and let $S=E$. Let the map $g$ : $E \rightarrow G$ be written as $g=\left(g_{1}, g_{2}\right)$, where $g_{i}\left(x_{1}, x_{2}\right)=f_{i}\left(x_{1}, x_{2}\right)-k_{i}\left(x_{1}, x_{2}\right)$ is such that $f_{i}\left(t_{1} x_{1}, t_{2} x_{2}\right)=t_{1}^{n_{i}} t_{2}^{m_{i}} f_{i}\left(x_{1}, x_{2}\right)$ for all $x_{1} \in E_{1}, x_{2} \in E_{2}, t_{1}, t_{2}>0$ and some nonnegative integers $n_{i}, m_{i}, i=1,2$. Assume that for any bounded set $M \subset E$ there exists $0 \leqslant K(M)<1$ such that $\alpha\left(k_{i}(M)\right) \leqslant K(M) \alpha\left(f_{i}(M)\right), i=1,2$ (this condition implies that $k_{i}$ and $f_{i}$ send bounded sets into bounded sets, unless $k_{i}$ is compact). In the case when $K(M)$ is not uniformly bounded by some constant $0 \leqslant K<1$, the map $f=\left(f_{1}, f_{2}\right)$ is assumed to be proper on bounded and closed sets of $E$. Furthermore assume that $g^{-1}(0) \subset B_{1}^{\varepsilon} \times B_{2}^{\varepsilon} \cup A$, where $B_{i}^{\varepsilon}=\left\{x_{i} \in E_{i}:\left\|x_{i}\right\|<r_{i}-\varepsilon\right\}, \varepsilon>0$, and $B_{i}$ will stand for $B_{i}=\left\{x_{i} \in E_{i}:\left\|x_{i}\right\|<r_{i}\right\}, r_{i}>0, i=1,2$, and $A \subset\left(E_{1} \backslash B_{1}^{\varepsilon}\right) \times\{0\}$ is a closed set such that if $\left(x_{1}, 0\right) \in A$, then $\left(t x_{1}, 0\right) \in A$ for any $t \geqslant 1$ (so that if $A \neq \varnothing$, then $A$ is unbounded $)$. Then:

(a) If $A \subset\left(E_{1} \backslash B_{1}\right) \times\{0\}$ and $K(M)<K<1$, then $g$ is 0 -epi on $E \backslash A$ if and only if $g$ is 0 -epi on $B_{1} \times B_{2}$. If $A=\varnothing$ and the map $g_{0} \equiv\left(k_{1}, k_{2}\right)$ is compact, then one may take $\varepsilon=0$ and $g$ is 0 -epi on $\bar{B}_{1} \times \bar{B}_{2}$. If $K=1$ and $f-(1-\tau) g_{0}$ is 0 -epi on $B_{1} \times B_{2}$ for $0 \leqslant \tau<\tau_{0}$, then $g$ is 0 -epi on $E \backslash A$, provided that the zeros of $f-$ $(1-\tau) g_{0}$ are in $B_{1}^{\varepsilon} \times B_{2}^{\varepsilon} \cup A$.

(b) If $g^{-1}(0) \cap\left(B_{1} \times B_{2} \backslash\left(B_{1} \times\{0\}\right)\right)$ is closed and $K<1$, then $g$ is 0-epi on $E \backslash\left(A \cup\left(\bar{B}_{1}^{\varepsilon} \times\{0\}\right)\right)$ if and only if $g$ is 0 -epi on $B_{1} \times B_{2} \backslash\left(A \cup\left(\bar{B}_{1}^{\varepsilon} \times\{0\}\right)\right)$. If $K=1$ and $f-(1-\tau) g_{0}$ is 0 -epi on $B_{1} \times B_{2} \backslash\left(A \cup\left(\bar{B}_{1}^{\varepsilon} \times\{0\}\right)\right)$ for $0 \leqslant \tau<\tau_{0}$, then $g$ is 0 -epi on $E \backslash\left(A \cup\left(\bar{B}_{1}^{\varepsilon} \times\{0\}\right)\right)$, provided that the zeros of $f-(1-\tau) g_{0}$ are in $B_{1}^{\varepsilon} \times B_{2}^{\varepsilon} \cup A$.

Proof. (Only if ). Since $g^{-1}(0)$ is closed and $g^{-1}(0) \cap(E \backslash A) \subset B_{1}^{\varepsilon} \times B_{2}^{\varepsilon}$, it follows that $g$ is admissible on $B_{1} \times B_{2}\left(\bar{B}_{1} \times \bar{B}_{2}\right.$ if $A=\varnothing$ and $\left.\varepsilon=0\right)$. In case (a), the set $g^{-1}(0) \cap(E \backslash A)$ is closed. In case (b), we have

$$
\begin{aligned}
g^{-1}(0) \cap\left(E \backslash\left(A \cup\left(\bar{B}_{1}^{\varepsilon} \times\{0\}\right)\right)\right) & =g^{-1}(0) \cap\left(B_{1}^{\varepsilon} \times B_{2}^{\varepsilon} \backslash\left(\bar{B}_{1}^{\varepsilon} \times\{0\}\right)\right) \\
& =g^{-1}(0) \cap\left(B_{1} \times B_{2} \backslash\left(B_{1} \times\{0\}\right)\right),
\end{aligned}
$$

which is closed by hypothesis. Hence, by normality, $g$ is admissible on

$$
E \backslash\left(A \cup\left(\bar{B}_{1}^{\varepsilon} \times\{0\}\right)\right) \text { and } B_{1} \times B_{2} \backslash\left(A \cup\left(\bar{B}_{1}^{\varepsilon} \times\{0\}\right)\right) .
$$

The implication now follows from Property 2.2 .

(If ). We shall consider first the case when $\varepsilon>0$. Assume that $g$ is not 0 -epi on $E \backslash A$ (on $E \backslash\left(A \cup\left(\bar{B}_{1}^{\varepsilon} \times\{0\}\right)\right)$ respectively). From Propositions 2.1 and 2.2 it 
follows that there exists an open and bounded set $V$, satisfying

$$
g^{-1}(0) \cap(E \backslash A) \subset V \subset \bar{V} \subset E \backslash A
$$

$\left(g^{-1}(0) \cap\left(E \backslash\left(A \cup\left(\bar{B}_{1}^{\varepsilon} \times\{0\}\right)\right)\right) \subset V \subset \bar{V} \subset E \backslash\left(A \cup\left(\bar{B}_{1}^{\varepsilon} \times\{0\}\right)\right)\right.$ respectively $)$, and such that $g$ is not 0 -epi on $\bar{V}$. Obviously, $\bar{V} \subset \tilde{B}_{1} \times \tilde{B}_{2}$, where $\tilde{B}_{i}=\left\{x_{i} \in E_{i}\right.$ : $\left.\left\|x_{i}\right\|<R_{i}\right\}, R_{i}>0, i=1,2$. Therefore, by Property 2.2, $g$ is not 0 -epi on

$$
\left(\tilde{B}_{1} \times \tilde{B}_{2}\right) \backslash A
$$

$\left(\left(\tilde{B}_{1} \times \tilde{B}_{2}\right) \backslash\left(A \cup\left(\bar{B}_{1}^{\varepsilon} \times\{0\}\right)\right)\right.$ respectively). For $K=1$, the map $f-(1-\tau) g_{0}$ is not 0 -epi for a sequence $\tau_{n} \rightarrow 0$ (see Corollary 2.4). On $B_{1}$ consider the scaling

$$
s(t) x_{1}=\left\{\begin{array}{l}
x_{1} \quad \text { if }\left\|x_{1}\right\| \leqslant r_{1}-\varepsilon, \\
\left(1+\frac{t\left(R_{1}-r_{1}\right)\left(\left\|x_{1}\right\|-\left(r_{1}-\varepsilon\right)\right)}{\varepsilon r_{1}}\right) x_{1} \quad \text { if } r_{1}-\varepsilon \leqslant\left\|x_{1}\right\|<r_{1} .
\end{array}\right.
$$

The scaling $s(t) x_{1}$ is a homeomorphism from $B_{1}$ into $\tilde{B}_{1}$, leaves $B_{1}^{\varepsilon}$ invariant and $s(1) x_{1}$ is onto $\tilde{B}_{1}$. Then it is easy to see that $g$ is 0 -epi on

$$
\left(\tilde{B}_{1} \times \tilde{B}_{2}\right) \backslash A
$$

$\left(\left(\tilde{B}_{1} \times \tilde{B}_{2}\right) \backslash\left(A \cup\left(\bar{B}_{1}^{\varepsilon} \times\{0\}\right)\right)\right.$ respectively), if and only if the map $g(s(1) \cdot, \cdot)$ is 0 -epi on $\left(B_{1} \times \tilde{B}_{2}\right) \backslash s(1)^{-1}(A)\left(\left(B_{1} \times \tilde{B}_{2}\right) \backslash\left(s(1)^{-1}(A) \cup\left(\bar{B}_{1}^{\varepsilon} \times\{0\}\right)\right)\right.$ respectively $)$. Consider the homotopy

$$
\begin{aligned}
& \left(\frac{g_{1}\left(s(t) x_{1}, x_{2}\right)}{s(t)^{n_{1}}}, \frac{g_{2}\left(s(t) x_{1}, x_{2}\right)}{s(t)^{n_{2}}}\right) \\
& \quad=f\left(x_{1}, x_{2}\right)-\left(\frac{k_{1}\left(s(t) x_{1}, x_{2}\right)}{s(t)^{n_{1}}}, \frac{k_{2}\left(s(t) x_{1}, x_{2}\right)}{s(t)^{n_{2}}}\right)
\end{aligned}
$$

defined on $\left(B_{1} \times \tilde{B}_{2}\right) \backslash s(1)^{-1}(A)\left(\left(B_{1} \times \tilde{B}_{2}\right) \backslash\left(s(1)^{-1}(A) \cup\left(\bar{B}_{1}^{\varepsilon} \times\{0\}\right)\right)\right.$ respectively). The set of zeros of the homotopy are those of $g$ for $\left\|x_{1}\right\| \leqslant r_{1}-\varepsilon$ and are contained in $s(1)^{-1}(A)$ for $x_{2}=0, r_{1}-\varepsilon \leqslant\left\|x_{1}\right\|$ since $s(t)$ is increasing in $t$ and from the construction of $A$, so that $s(t)^{-1}(A) \subset s(1)^{-1}(A)$. Hence, on the above sets, the zeros are not moved.

Let $M$ be a bounded subset of $B_{1} \times \tilde{B}_{2}$ and let $n=\max \left\{n_{1}, m_{1}, n_{2}, m_{2}\right\}$. Choose an integer $N$ such that $N>K^{1 / n}\left(R_{1}-r_{1}\right) /\left(1-K^{1 / n}\right) r_{1}$. Then, for any integer $j=0,1, \ldots, N-1$, one has that $0 \leqslant s((i+1) / N)-s(i / N) \leqslant\left(R_{1}-r_{1}\right) / N r_{1}$ and hence,

$$
K\left(\frac{s((i+1) / N)}{s(i / N)}\right)^{n}<1 .
$$

Write $M \times[0,1]$ as $M_{\varepsilon} \times[0,1] \bigcup_{j=0}^{N-1} N_{\varepsilon} \times I_{j}$, where $M_{\varepsilon}=M \cap\left(B_{1}^{\varepsilon} \times E_{2}\right), N_{\varepsilon}=M$ $\cap\left(\left(B_{1} \backslash B_{1}^{\varepsilon}\right) \times E_{2}\right)$ and $I_{j}=[j / N,(j+1) / N]$. Then,

$$
\begin{aligned}
\alpha\left(\left(\frac{k_{1}(s(\cdot) \cdot, \cdot)}{s(\cdot)^{n_{1}}}\right)\right. & (M \times[0,1])) \\
= & \left.\max \left\{\alpha\left(k_{1}(\cdot, \cdot)\left(M_{\varepsilon}\right)\right), \alpha\left(\frac{k_{1}(s(\cdot) \cdot, \cdot)}{s(\cdot)^{n_{1}}}\right)\left(N_{\varepsilon} \times I_{j}\right)\right)\right\}
\end{aligned}
$$


and

$$
\begin{array}{r}
\alpha\left(\left(\frac{k_{1}(s(\cdot) \cdot, \cdot)}{s(\cdot)^{n_{1}}}\right)\left(N_{\varepsilon} \times I_{j}\right)\right)=\alpha\left(\overline{\operatorname{co}}\left(\frac{k_{1}(s(\cdot) \cdot, \cdot)}{s(\cdot)^{n_{1}}}\left(N_{\varepsilon} \times I_{j}\right) \cup\{0\}\right)\right) \\
\leqslant \alpha\left(\frac{k_{1}(s(\cdot) \cdot, \cdot)}{s(j / N)^{n_{1}}}\left(N_{\varepsilon} \times I_{j}\right)\right)=\frac{1}{s(j / N)^{n_{1}}} \alpha\left(k_{1}(\cdot, \cdot)\left(N_{j}\right)\right),
\end{array}
$$

where $N_{j}=\left\{\left(s(t) x_{1}, x_{2}\right):\left(x_{1}, x_{2}\right) \in N_{\varepsilon}\right.$ and $\left.j / N \leqslant t \leqslant(j+1) / N\right\}$. Now,

$$
\begin{aligned}
\frac{1}{s(j / N)^{n_{1}}} \alpha\left(k_{1}(\cdot, \cdot)\left(N_{j}\right)\right) & \leqslant \frac{1}{s(j / N)^{n_{1}}} K \alpha\left(f_{1}(\cdot, \cdot)\left(N_{j}\right)\right) \\
& =\frac{K}{s(j / N)^{n_{1}}} \alpha\left(s(\cdot)^{n_{1}} f_{1}(\cdot, \cdot)\left(N_{\varepsilon} \times I_{j}\right)\right) \\
& \leqslant \frac{s((j+1) / N)^{n_{1}}}{s(j / N)^{n_{1}}} K \alpha\left(f_{1}(\cdot, \cdot)\left(N_{\varepsilon}\right)\right) \\
& \leqslant K\left(\frac{R_{1}-r_{1}}{N r_{1}}+1\right)^{n} \alpha\left(f_{1}(\cdot, \cdot)(M)\right) .
\end{aligned}
$$

Then,

$$
\alpha\left(\left(\frac{k_{1}(s(\cdot) \cdot, \cdot)}{s(\cdot)^{n_{1}}}\right)(M \times[0,1])\right) \leqslant K(N) \alpha\left(f_{1}(\cdot, \cdot)(M)\right)
$$

for any large $N$. Sending $N$ to infinity one obtains the constant $K<1$. Since the same estimate holds for $k_{2}$, one gets that

$$
\begin{aligned}
\alpha\left(\left(\frac{k_{1}(s(\cdot) \cdot, \cdot)}{s(\cdot)^{n_{1}}},\right.\right. & \left.\left.\frac{k_{2}(s(\cdot) \cdot, \cdot)}{s(\cdot)^{n_{2}}}\right)(M \times[0,1])\right) \\
& \leqslant K \max \left\{\alpha\left(f_{1}(\cdot, \cdot)(M)\right), \alpha\left(f_{2}(\cdot, \cdot)(M)\right)\right\} .
\end{aligned}
$$

However, for any set $C \subset G$ one has that $C \subset P_{1} C \times P_{2} C$, where $P_{i}$ is the projection onto $G_{i}, \quad i=1,2$. Hence, $\alpha(C) \leqslant \alpha\left(P_{1} C \times P_{2} C\right) \leqslant \max \left\{\alpha\left(P_{1} C\right), \alpha\left(P_{2} C\right)\right\}$ (see [FMV,I, p. 238]) and since $\alpha\left(P_{1} C\right) \leqslant \alpha\left(P_{1}\right) \alpha(C) \leqslant \alpha(C)$, then

$$
\alpha(C)=\max \left\{\alpha\left(P_{1} C\right), \alpha\left(P_{2} C\right)\right\} .
$$

Here, taking $C=\left(f_{1}, f_{2}\right)(M)=f(M)$ one obtains that the homotopy is $K$-setcontractive with respect to $f$.

From Corollaries 2.1, 2.3 and 2.4 it follows that $g$ is not 0 -epi on

$$
\left(B_{1} \times \tilde{B}_{2}\right) \backslash s(1)^{-1}(A),
$$

$\left(\left(B_{1} \times \tilde{B}_{2}\right) \backslash\left(s(1)^{-1}(A) \cup\left(\bar{B}_{1}^{\varepsilon} \times\{0\}\right)\right)\right.$ respectively) if $K<1$, and there is a sequence $\tau_{n} \rightarrow 0$ such that $f-\left(1-\tau_{n}\right) g_{0}$ is not 0 -epi on those sets for $K=1$. Since the zeros of $\left.g\right|_{B_{1} \times \tilde{B}_{2}}$ are in $B_{1}^{\varepsilon} \times B_{2}^{\varepsilon}$ in case (a) and in $\left(B_{1}^{\varepsilon} \times B_{2}^{\varepsilon}\right) \cup\left(A \cap\left(B_{1} \times\{0\}\right)\right)$ in case (b), in which case $A \cap\left(B_{1} \times\{0\}\right) \subset s(1)^{-1}(A)$, then, from Property 2.2 , it follows that $g$ is not 0 -epi on $B_{1} \times \tilde{B}_{2}\left(\left(B_{1} \times \tilde{B}_{2}\right) \backslash\left(A \cup\left(\bar{B}_{1}^{\varepsilon} \times\{0\}\right)\right)\right.$ respectively $)$ for $K<1, f-\left(1-\tau_{n}\right) g_{0}$ for $K=1$ and $\tau_{n}$ close to 0 (notice that from the 
properness of $f$ it follows that the zeros of the last map are close to those of $f-g_{0}$ for $\tau_{n}$ close to 0 ; see the proof of Corollary 2.4).

If $A=\varnothing, \varepsilon=0$ and $k_{i}$ is compact, $i=1,2$, then the same argument with $\tilde{s}(t)=1+t\left(R_{1}-r_{1}\right) / r_{1}$, will show that $g$ is not 0 -epi on $\tilde{B}_{1} \times \overline{\tilde{B}}_{2}$ if and only if the map $g(\tilde{s}(1) \cdot, \cdot)$ is not 0 -epi on $\bar{B}_{1} \times \overline{\tilde{B}}_{2}$. The homotopy defined as above, with $s(t)$ replaced by $\tilde{s}(t)$, but only on $\partial\left(B_{1} \times \tilde{B}_{2}\right)$, will give that $g$ is not 0 -epi on $\bar{B}_{1} \times \overline{\tilde{B}}_{2}$ from Corollary 2.1(b).

Finally, the scaling in the $B_{2}$ direction, with all the indices 1 replaced by 2 in the expression for $s(t)$, will contradict the hypothesis. Q.E.D.

The following consequence of Proposition 4.1 shows that, under certain extendibility properties of $k_{1}$ (or $k_{2}$ ), the map $g$ need not be defined on the whole Banach space $E$.

Corollary 4.1. Let $U \subset E$ be an open subset such that $\bar{B}_{1} \times \bar{B}_{2} \subset U$ and let $g$ : $U \rightarrow G$ have the same properties of noncompactness, homogeneity and zeros as in Proposition 4.1, relative to $U$. Assume, in addition, that either $k_{1}$, or $k_{2}\left(\right.$ say $\left.k_{2}\right)$ has an extension to $E$ such that the corresponding $g_{2}\left(x_{1}, x_{2}\right)=f_{2}\left(x_{1}, x_{2}\right)-k_{2}\left(x_{1}, x_{2}\right)$ (here $f_{2}$ is extended by homogeneity) has, on $E$, the same properties of noncompactness and has all its zeros, either in some open and bounded subset $V_{1}$ such that $\bar{B}_{1} \times \bar{B}_{2} \subset V_{1} \subset$ $\bar{V}_{1} \subset U$, or in A. Then, the conclusions of (a) and (b) in Proposition 4.1 are valid, provided one replaces $E$ by $U$.

Proof. (Only if ). As in Proposition 4.1, replacing $E$ by $U$.

(If). Assume that $g$ is not 0 -epi on $U \backslash A\left(U \backslash\left(A \cup\left(\bar{B}_{1}^{\varepsilon} \times\{0\}\right)\right)\right.$ respectively). Again, there is an open and bounded set $V$ satisfying $g^{-1}(0) \cap(U \backslash A) \subset V \subset \bar{V} \subset$ $U \backslash A\left(g^{-1}(0) \cap\left(U \backslash\left(A \cup\left(\bar{B}_{1}^{\varepsilon} \times\{0\}\right)\right)\right) \subset V \subset \bar{V} \subset U \backslash\left(A \cup\left(\bar{B}_{1}^{\varepsilon} \times\{0\}\right)\right)\right.$ respectively) and such that $g$ is not 0 -epi on $\bar{V}$. Let $\varphi: E \rightarrow[0,1]$ be an Urysohn's function with $\varphi\left(\bar{V} \cup \bar{V}_{1}\right)=1$, having bounded support contained in $U$. Consider $\tilde{g}\left(x_{1}, x_{2}\right)=$ $f\left(x_{1}, x_{2}\right)-\tilde{g}_{0}\left(x_{1}, x_{2}\right)$, defined on $E$ as $\left(f_{1}\left(x_{1}, x_{2}\right)-\varphi\left(x_{1}, x_{2}\right) k_{1}\left(x_{1}, x_{2}\right)\right.$, $\left.g_{2}\left(x_{1}, x_{2}\right)\right)$, where $f_{1}$ has been extended by homogeneity to $E$. Clearly, the conditions on the measure of noncompactness are conserved. If $K=1$, then $f$ is proper on bounded and closed subsets of $E$ by the homogeneity and the zeros of $\tilde{g}$ are those of $g$ in $\bar{V} \cup \bar{V}_{1}$ and contained in $A$ outside $V_{1}$. Since $\tilde{g}=g$ on $\bar{V}$, then Proposition 4.1 will give that $\tilde{g}$ is not 0 -epi on $B_{1} \times B_{2}, \bar{B}_{1} \times \bar{B}_{2}\left(\left(B_{1} \times B_{2}\right) \backslash\left(A \cup\left(\bar{B}_{1}^{\varepsilon} \times\{0\}\right)\right)\right.$ or $f-(1-\tau) \tilde{g}_{0}$ respectively), but there $g=\tilde{g}$. Q.E.D.

Note that Corollary 4.1 represents a substitute for the excision property of degree theory. Actually, if one of the classical degree theories applies, then one may replace $B_{1} \times B_{2}$ by an arbitrary open set containing the zeros of the map $g$.

The following consequence of Theorem 3.1 and Corollary 4.1 will be applied to obtain both the continuation principle and the global implicit function theorem.

Corollary 4.2. Let $U$ and $g$ be as in Corollary 4.1 and satisfy the hypotheses of Proposition 4.1(a), with $\left(g_{1}, g_{2}\right)$ 0-epi on $B_{1} \times B_{2}$ (on $\bar{B}_{1} \times \bar{B}_{2}$ if $A=\varnothing, g_{0}$ is compact, $\varepsilon=0 ; f-(1-\tau) g_{0} 0$-epi on $B_{1} \times B_{2}$ if $\left.K=1\right)$. Let $S=g_{1}^{-1}(0)$. Assume that $g_{2}$ is sectionally proper and sectionally bounded on closed (in $E$ ) and bounded 
subsets of $S \backslash A$. Then, there is a minimal subset $\Sigma$ of $S \backslash A$ such that $g_{2}$ is sectionally 0-epi on $\Sigma \cap(U \backslash(A \cap \bar{\Sigma}))$ and $\Sigma$ has all of the properties listed in Theorem 3.1.

Proof. From Corollary 4.1 we have that $\left(g_{1}, g_{2}\right)$ is 0 -epi on $U \backslash A$ and, from Property 2.9, $g_{2}$ is 0 -epi on $S \cap(U \backslash A)$. Let $W=U \backslash(A \cap \bar{S}), \tilde{A}=A \backslash \bar{S}$. Then $W$ is open, $\tilde{A}$ is closed in $W, \bar{S} \cap \tilde{A}=\varnothing$ and $W \backslash \tilde{A}=U \backslash A$. From Property 2.8 the map $g_{2}$ is 0 -epi on $S \cap W$ and, by Property 2.6, on $(S \backslash A) \cap(U \backslash(A \cap \bar{S}))$. Applying Theorem 3.1 one gets the set $\Sigma$ such that $g_{2}$ is sectionally 0-epi on $\Sigma \cap(U \backslash(A \cap \bar{S}))$. Again, by Property 2.8 (see §2D), taking $W_{1}=U \backslash(A \cap \bar{\Sigma})$, $A_{1}=(A \cap \bar{S}) \backslash \bar{\Sigma}$, we get that $g_{2}$ is sectionally 0 -epi on $\Sigma \cap(U \backslash(A \cap \bar{\Sigma}))$. The fact that $\Sigma$ is also minimal for $U \backslash(A \cap \bar{\Sigma})$ comes from the reverse implication in Property 2.8. Q.E.D.

The following corollary will be used to obtain our global bifurcation result.

COROllary 4.3. Let $U$ and $g$ be as in Corollary 4.1 and satisfy the hypotheses of Proposition 4.1(b), with $\left(g_{1}, g_{2}\right)$ 0-epi on $B_{1} \times B_{2} \backslash\left(A \cup\left(\bar{B}_{1}^{\varepsilon} \times\{0\}\right)\right)\left(f-(1-\tau) g_{0}\right.$, if $K=1)$. Let $S=g_{1}^{-1}(0)$. Assume that $g_{2}$ is sectionally proper and sectionally bounded on closed (in $E$ ) and bounded subsets of $S \backslash\left(A \cup\left(\bar{B}_{1}^{\varepsilon} \times\{0\}\right)\right)$. Then there is a minimal subset $\Sigma$ of $S \backslash\left(A \cup\left(\bar{B}_{1}^{\varepsilon} \times\{0\}\right)\right)$ such that $g_{2}$ is sectionally 0-epi on $\Sigma \cap\left(U \backslash\left(A \cup\left(\bar{B}_{1}^{\varepsilon} \times\{0\}\right) \cap \bar{\Sigma}\right)\right)$ and $\Sigma$ has all of the properties listed in Theorem 3.1.

Proof. The same as in Corollary 4.2 replacing $A$ everywhere by $A \cup\left(\bar{B}_{1}^{\varepsilon} \times\{0\}\right)$. Q.E.D.

We now give an extension of Corollaries 4.1, 4.2 and 4.3 to the context of $A$-proper 0-epi maps on $S \cap U$.

Corollary 4.4. Assume that the Banach spaces $E$ and $G$ have projectional schemes as in Remark 2.9. Assume that $g=\left(g_{1}, g_{2}\right)$ satisfies the assumptions (2) and (3) of Remark 2.9 and that $g^{-1}(0) \subset B_{1}^{\varepsilon} \times B_{2}^{\varepsilon} \cup A, g_{2}^{-1}(0) \subset V_{1} \cup A, g_{n}^{-1}(0) \subset\left(B_{1}^{\varepsilon} \times B_{2}^{\varepsilon}\right.$ $\cup A) \cap E_{n}, g_{2, n}^{-1}(0) \subset\left(V_{1} \cup A\right) \cap E_{n}$, where $g_{2}$ is defined on $E$ and $A, \varepsilon, V_{1}$ are as in Corollary 4.1 (notice that neither homogeneity, compactness, nor continuity on $g$ are assumed). Then:

(a) If either $A \subset\left(E_{1} \backslash B_{1}\right) \times\{0\}$, or $A \subset\left(E_{1} \backslash \bar{B}_{1}\right) \times\{0\}$ and $\varepsilon=0$, then $g$ is $A$-proper 0-epi on $U \backslash A$ if and only if $g$ is A-proper 0-epi on $B_{1} \times B_{2}$. If $g$ is A-proper 0 -epi on $B_{1} \times B_{2}$ and, moreover, if $S=g_{1}^{-1}(0)$ is closed in $U, g_{2}$ is continuous on $S$, sectionally proper and sectionally bounded on closed (in $E$ ) and bounded subsets of $S \backslash A$, then one has the same conclusions as in Corollary 4.2.

(b) If

$$
\begin{aligned}
g_{n}^{-1}(0) \cap & \left(B_{1} \times B_{2} \backslash\left(B_{1} \times\{0\}\right)\right) \\
& \subset V_{0} \cap E_{n} \subset \bar{V}_{0} \cap E_{n} \subset\left(B_{1} \times B_{2} \backslash\left(B_{1} \times\{0\}\right)\right) \cap E_{n},
\end{aligned}
$$

for $n$ large, then $g$ is A-proper 0-epi on $U \backslash\left(A \cup\left(\bar{B}_{1}^{\varepsilon} \times\{0\}\right)\right)$ if and only if $g$ is $A$-proper 0 -epi on $B_{1} \times B_{2} \backslash\left(A \cup\left(\bar{B}_{1}^{\varepsilon} \times\{0\}\right)\right)$. If $g$ is $A$-proper 0 -epi on $B_{1} \times$ $B_{2} \backslash\left(A \cup\left(\bar{B}_{1}^{\varepsilon} \times\{0\}\right)\right)$ and, moreover, if $S=g_{1}^{-1}(0)$ is closed in $U, g_{2}$ is continuous on $S$, sectionally proper and sectionally bounded on closed (in $E$ ) and bounded subsets of $S \backslash\left(A \cup\left(\bar{B}_{1}^{\varepsilon} \times\{0\}\right)\right)$, then one has the same conlusions as in Corollary 4.3. 
Proof. If $\varepsilon=0$ and $A \subset\left(E \backslash \bar{B}_{1}\right) \times\{0\}$, since $g_{n}^{-1}(0) \cap\left(\bar{B}_{1} \times \bar{B}_{2}\right)$ is compact and $A$ is closed, the existence of an $\varepsilon_{n}$ with the properties of Proposition 4.1 is assured for each $n$ (for the other cases $\varepsilon_{n}=\varepsilon$ ). By Corollary 4.1 the map $g_{n}$ is 0-epi on $(U \backslash A) \cap E_{n}$ if and only if $g_{n}$ is 0-epi on $\left(B_{1} \times B_{2}\right) \cap E_{n}$ and $g_{n}$ is 0-epi on $\left(U \backslash\left(A \cup \bar{B}_{1}^{\varepsilon} \times\{0\}\right)\right) \cap E_{n}$ if and only if $g_{n}$ is 0 -epi on $\left(B_{1} \times B_{2} \backslash\left(A \cup \bar{B}_{1}^{\varepsilon} \times\{0\}\right)\right)$ $\cap E_{n}$. The results analogous to Corollary 4.1 follow from the control on the zeros of $g$ and $g_{n}$. If $S$ is closed in $U$ and $g_{2}$ is continuous on $S$, then, from Remark 2.9, the map $g_{2}$ is 0 -epi on $S \cap(U \backslash A)$, respectively on $S \cap\left(U \backslash\left(A \cup\left(\bar{B}_{1}^{\varepsilon} \times\{0\}\right)\right)\right)$. The last part of the conclusion follows as in Corollaries 4.2 and 4.3. Q.E.D.

B. Application to continuation and global implicit function theorem. Let $U$ be an open subset of $E \times \Lambda$, where $E$ and $\Lambda$ are Banach spaces. Here $\Lambda$ is the parameter space which may be infinite dimensional: if this is the case, let $\left\{\Lambda_{M}\right\}$ be the family of sections of $\Lambda$ given by all finite dimensional subspaces of $\Lambda$.

Assume that $0 \in \bar{B}_{1} \times \bar{B}_{2} \subset U$ and that $g_{1}: U \rightarrow G$ is of the form $g_{1}(x, \lambda)=$ $f_{1}(x)-k_{1}(x, \lambda)$, with $f_{1}(t x)=t^{n} f_{1}(x)$ for $t>0$ and some $n>0$.

Assume also that:

(1) $f_{1}$ is proper on $\bar{B}_{1}$.

(2) If $C$ is a bounded subset of $U_{M} \equiv U \cap\left(E \times \Lambda_{M}\right)$, then $\alpha\left(k_{1}(C)\right) \leqslant$ $K_{M}(C) \alpha\left(f_{1}(C)\right)$, with $K_{M}(C)<K \leqslant 1$, for large $M$ (unless $k_{1}$ is a compact map, $f_{1}$ and $k_{1}$ send bounded sets into bounded sets).

(3) $g_{1}(x, 0) \neq 0$ for all $x \in \partial B_{1}$.

With this preparation we have the following.

THEOREM 4.1. Let $U$ and $g_{1}$ be as above and assume that $\left(g_{1}(x, 0), \lambda_{M}\right)$ is 0-epi on $B_{1} \times\left(B_{2} \cap \Lambda_{M}\right)$ for large $M$ (the map $\left(f_{1}(x)-(1-\tau) k_{1}(x, 0), \lambda_{M}\right)$ is 0-epi on the same set if $K=1$ for $\left.0 \leqslant \tau<\tau_{0}\right)$. Then, if $S=g_{1}^{-1}(0)$ and $A=\left\{(x, 0):\|x\| \geqslant r_{1}\right\}$, the set $S \backslash A$ has a minimal closed subset $\Sigma$ such that $g_{2}(x, \lambda) \equiv \lambda$ is sectionally 0-epi on $\Sigma \cap(U \backslash(A \cap \bar{\Sigma}))$ with the following properties.

(a) $\Sigma$ intersects the fiber $\{\lambda=0\}$ inside $B_{1}$ and, if $g_{1}(x, \lambda) \neq 0$ on $\partial B_{1} \times \bar{B}_{2}\left(r_{2}^{\prime}\right)$ for some $r_{2}^{\prime} \leqslant r_{2}$, then $\Sigma$ intersects the fiber $\left\{\lambda=\lambda_{0}\right\}$ inside $B_{1}$ for any $\lambda_{0}$ with $\left|\lambda_{0}\right|<r_{2}^{\prime}$.

(b) $\Sigma \cap \Lambda_{M}$ for $M$ large is either unbounded, or $\left(\overline{\Sigma \cap \Lambda_{M}}\right) \cap \partial U_{M} \neq \varnothing$, or $\left(\overline{\Sigma \cap \Lambda_{M}}\right) \cap A \neq \varnothing$.

(c) If $\Sigma=\Sigma_{1} \cup \Sigma_{2}$, with $\Sigma_{1}, \Sigma_{2}$ proper and closed subsets of $\Sigma$, then $\operatorname{dim}\left(\Sigma_{1} \cap \Sigma_{2}\right)$ $\geqslant \operatorname{dim} \Lambda-1$. In particular, $\Sigma$ is connected and $\bar{\Sigma}$ has dimension at each point at least $\operatorname{dim} \Lambda$ (for points of $\bar{\Sigma} \cap \partial U$ and $\bar{\Sigma} \cap A$ this has to be taken in the sense of Theorem 3.1).

(d) If $\bar{\Sigma} \cap A=\varnothing$ and the hypotheses for $g_{1}$ hold with $U$ replaced by $\bar{U}$, then $\lambda$ is sectionally 0-epi on $\bar{\Sigma} \cap \bar{U}, \bar{\Sigma}$ is minimal and $\operatorname{dim}(\bar{\Sigma} \cap \partial V) \geqslant \operatorname{dim} \Lambda-1$ for any bounded open set $V$ with $B_{1} \times B_{2} \subset V \subset U$ and for $V=U$ if $\bar{\Sigma}$ is bounded. Finally, if $\bar{\Sigma} \cap\left(E \times\left[0, \lambda_{0}\right]\right)$ is bounded for any bounded $\lambda_{0}$ and if $\operatorname{dim}(\bar{\Sigma} \cap \partial U)<\operatorname{dim} \Lambda-1$, then $\bar{\Sigma}$ covers $\Lambda$.

Proof. Note first that $f_{1}$ is proper on closed and bounded subsets of $E$ so that, from hypothesis (2), the map $g_{1}$ is proper on closed (in $E$ ) bounded subsets of $U_{M}$. In particular the intersection of $S$ with any closed (in $E$ ) bounded subset of $U_{M}$ is 
compact. From this fact and property (3), it follows that there is an $\varepsilon>0$ such that $\left(g_{1}(x, \lambda), \lambda\right)^{-1}(0) \subset B_{1}^{\varepsilon} \times B_{2}^{\varepsilon} \cup A$. Clearly $\lambda$ is sectionally proper and sectionally bounded on closed (in $E$ ) bounded subsets of $S$.

Now $\left(g_{1}\left(x, \lambda_{M}\right), \lambda_{M}\right)$ is 0 -epi on $B_{1} \times\left(B_{2} \cap \Lambda_{M}\right)$ if and only if $\left(g_{1}(x, 0), \lambda_{M}\right)$ is 0 -epi on this set, since the homotopy $\left(g_{1}\left(x, t \lambda_{M}\right), \lambda_{M}\right)$ is admissible

$$
\begin{aligned}
\alpha\left(k_{1}(\cdot, \cdot)(C \times I)\right) & =\alpha\left(k_{1}(\cdot, \cdot)\left(C_{I}\right)\right) \\
& \leqslant K_{M}\left(C_{I}\right) \alpha\left(f_{1}\left(C_{I}\right)\right)=K_{M}\left(C_{I}\right) \alpha\left(f_{1}(C)\right),
\end{aligned}
$$

where $\left.C_{I}=\left\{\left(x, t \lambda_{M}\right):\left(x, \lambda_{M}\right) \in C\right\}\right)$. (Note also that, since $g_{1}$ is proper on $\bar{B}_{1} \times\left(\bar{B}_{2} \cap \Lambda_{M}\right)$, from Proposition 2.3 , the map $\left(g_{1}(x, 0), \lambda_{M}\right)$ is 0 -epi on $B_{1} \times\left(B_{2}\right.$ $\left.\cap \Lambda_{M}\right)$ if and only if it is 0-epi on $\bar{B}_{1} \times\left(\bar{B}_{2} \cap \Lambda_{M}\right)$.) Thus, from Corollary 4.1, the map $\left(g_{1}, \Lambda_{M}\right)$ is 0-epi on $(U \backslash A) \cap\left(E \times \Lambda_{M}\right)$ and, from Property 2.9 we obtain that $\lambda_{M}$ is 0 -epi on $\left(S \cap \Lambda_{M}\right) \cap\left(U_{M} \backslash A\right)$ and hence on $\left(S \cap \Lambda_{M}\right) \cap(U \backslash A)$ (compact maps on $U \backslash A$ give compact maps on $U_{M} \backslash A$ and the solutions of $\lambda_{M}-h\left(x, \lambda_{M}\right)=0$ have to be on $\left.S \cap \Lambda_{M}\right)$. Thus $\lambda$ is sectionally 0-epi on $S \cap$ $(U \backslash A)$, then from Corollary 4.2 and Theorem 3.1, one gets all of the properties of the theorem except the second part of (a).

If $g_{1}(x, \lambda) \neq 0$ on $\partial B_{1} \times \bar{B}_{2}\left(r_{2}^{\prime}\right)$, then from the compactness of $S \cap \Lambda_{M} \cap\left(\bar{B}_{1} \times\right.$ $\left.\bar{B}_{2}\left(r_{2}^{\prime}\right)\right)$ one gets $\varepsilon_{M}$ such that $g_{1}\left(x, \lambda_{M}\right) \neq 0$ on $\left(\bar{B}_{1} \backslash \bar{B}_{1}^{\varepsilon_{M}}\right) \times\left(\bar{B}_{2}\left(r_{2}^{\prime}\right) \cap \Lambda_{M}\right)$. Then let $\lambda_{0}$, with $\left|\lambda_{0}\right|<r_{2}^{\prime}$, be fixed so that $\lambda_{0} \in \Lambda_{M}$ for $M$ large enough. Let $\varphi: \mathbf{R}^{+} \rightarrow \mathbf{R}^{+}$ be a nonincreasing function with $\varphi(r)=1$ for $0 \leqslant r \leqslant r_{1}-\varepsilon_{M}$ and $\varphi(r)=0$ for $r \geqslant r_{1}$. Consider the finite dimensional homotopy $\lambda-t \varphi(\|x\|) \lambda_{0}$ which is admissible by construction. Therefore $\lambda-\varphi(\|x\|) \lambda_{0}$ is sectionally 0 -epi on $\Sigma$ and, from Property 2.1 , the set $\Sigma$ must intersect the zero set of $\lambda-\varphi(\|x\|) \lambda_{0}$ in $U \backslash(A \cap \bar{\Sigma})$, hence in $B_{1}^{\varepsilon_{M}}$, where $\varphi(\|x\|)=1$. Q.E.D.

In the context of $A$-proper 0 -epi maps we have the following.

Corollary 4.5. Assume that $E$ and $F$ have projectional schemes as in Remark 2.9 (so that one can give natural schemes on $E \times \Lambda_{M}$ and $F \times \Lambda_{M}$ by adding the identity on the second component $)$. Assume that $\left(g_{1}\left(x, \lambda_{M}\right), \lambda_{M}\right)$ satisfies assumptions (2) and (3) of Remark 2.9. Suppose that:

(1) $g_{1}^{-1}(0) \cap \Lambda_{M}$ intersected with any bounded and closed (in E) subset of $U$ is compact for large $M$ (this is automatically true if $g_{1}$ is continuous on $U_{M}$ ).

(2) $g_{1}(x, 0) \neq 0$ on $\partial B_{1}$.

(3) $\left(g_{1}(x, 0), \lambda_{M}\right)$ is A-proper 0-epi on $B_{1} \times B_{2}$.

Then $S \backslash A$ has a minimal closed subset $\Sigma$ such that $\lambda$ is sectionally 0-epi on $\Sigma \cap(U \backslash(A \cap \bar{\Sigma}))$ with the properties of Theorem 4.1 .

Proof. Note first that the local compactness of $g_{1}^{-1}(0) \cap \Lambda_{M}$ for a continuous $A$-proper map $g_{1}$ is well known. From assumptions (1) and (2) there is an $\varepsilon>0$ such that $g_{1}(x, 0) \neq 0$ for $r_{1}-\varepsilon \leqslant\|x\| \leqslant r_{1}+\varepsilon$. Let $A_{\varepsilon}=\left\{(x, 0):\|x\| \geqslant r_{1}+\varepsilon\right\}$. Then $g\left(x, \lambda_{M}\right)=\left(g_{1}\left(x, \lambda_{M}\right), \lambda_{M}\right)$ has its zeros in $\left(B_{1}^{\varepsilon} \times B_{2}^{\varepsilon} \cup A_{\varepsilon}\right) \cap\left(E \times \Lambda_{M}\right)$ as well as $g_{n}\left(x, \lambda_{M}\right)=\left(g_{1, n}\left(x, \lambda_{M}\right), \lambda_{M}\right)$ for $n$ large enough, since if $g_{1, n}\left(x_{n}, 0\right)=0$ for $r_{1}-\varepsilon \leqslant\|x\| \leqslant r_{1}+\varepsilon$, then from Property (3) of Remark 2.9 one would have $g_{1}(x, 0)=0$ for some $x$ in this annulus. The rest of the proof follows from Corollary 
4.4(a), modifying the argument for an infinite dimensional parameter space as in the proof of Theorem 4.1. Q.E.D.

We shall give below equivalent conditions for $\left(g_{1}(x, 0), \lambda_{M}\right)$ to be 0 -epi on $\bar{B}_{1} \times\left(\bar{B}_{2} \cap \Lambda_{M}\right)$ when $g_{1}$ belongs to a more restricted class of maps.

Proposition 4.2. Assume that $g_{1}(x, 0)$ is of the form $x-k_{1}(x)$ from $\bar{B}_{1}$ into $E^{\infty-n}$, where $E^{\infty-n}$ is an $n$-codimensional subspace of $E$ and $k_{1}$ is compact. Then $\left(g_{1}(x, 0)\right.$, $\left.\lambda_{M}\right)$ is 0-epi on $\bar{B}_{1} \times\left(\bar{B}_{2} \cap \Lambda_{M}\right)$ if and only if the Mth suspension of the homotopy class of $\left.g_{1}(x, 0)\right|_{\partial B_{1}}$ is nontrivial (the stable homotopy class if $\operatorname{dim} E=+\infty$ ), where $M=\operatorname{dim} \Lambda_{M}$. In particular, if $E^{\infty-n}=E$, if and only if the Leray-Schauder degree of $g_{1}(x, 0)$ on $B_{1}$ is nonzero.

PROOF. Here $\lambda_{M}$ acts as a suspension and the proposition follows from [GG or I,I, p. 74]. Q.E.D.

REMARK 4.1. Similar statements hold if $g_{1}(x, 0)=x-k_{1}(x)$ with $k_{1}$ condensing, $E^{\infty-n}=E$, or if $g_{1}$ is $A$-proper with $\operatorname{dim} Q_{n} F=\operatorname{dim} P_{n} E$ and the respective degrees. Also, if $g_{1}$ is $A$-proper, one may ask that the $M$ th suspension of the homotopy classes of $g_{1, n}$ be nontrivial for an infinite number of $n$ 's. As noted above, if $E^{\infty-n}=E$, one may replace $B_{1}$ by any bounded open set in $E$, since then one may use the additivity property of the classical degree theory.

The following result contains the application to the global implicit function theorem.

Proposition 4.3. Assume that $f_{1}$ is a linear Fredholm operator of nonnegative index and that $k_{1}(x, 0)=o(\|x\|)$ for $x$ close to zero (hence $\left.g_{1}(0,0)=0\right)$ and $Q k_{1}$ is condensing with respect to $f_{1}$ on $\bar{B}_{1}$, where $Q$ is a projection from $F$ onto Range $f_{1}$. Assume that for all $r_{1}$ small enough $(I-Q) k_{1}\left(x_{1}+x_{2}\right) \neq 0$ for $x_{1} \in \operatorname{Ker} f_{1}$ with $\left\|x_{1}\right\|=r_{1}$ and $x_{2}$ belonging to a complement of $\operatorname{Ker} f_{1}$ with $\left\|x_{2}\right\|=o\left(r_{1}\right)$. Then, $\left(g_{1}(x, 0), \lambda_{M}\right)$ is 0-epi on $\bar{B}_{1} \times\left(\bar{B}_{2} \cap \Lambda_{M}\right)$ if and only if $(I-Q) k_{1}\left(x_{1}\right)$ as a mapping from $S^{d-1} \equiv\left\{x \in \operatorname{Ker} f_{1}:\left\|x_{1}\right\|=r_{1}\right\}, d=\operatorname{dim} \operatorname{Ker} f_{1}$, onto $\mathbf{R}^{d^{*}} \backslash\{0\}, d^{*}=$ codim Range $f_{1}$, has a nontrivial stable homotopy class (the $n+M$ suspended homotopy class if $n \equiv \operatorname{dim}$ Range $\left.f_{1}<+\infty\right)$. If $f_{1}$ is one-to-one, then $\left(g_{1}(x, 0), \lambda_{M}\right)$ is 0 -epi on $\bar{B}_{1} \times\left(\bar{B}_{2} \times \Lambda_{M}\right)$.

Proof. Note first that if $f_{1}$ is a linear operator which is proper on $\bar{B}_{1}$, then $f_{1}$ is semi-Fredholm with finite dimensional kernel and closed range, so that assumption (1) of Proposition 4.1 gives a part of the above hypothesis. Write then $x=x_{1}+x_{2}$, with $x_{1} \in \operatorname{Ker} f_{1}$ and $x_{2}$ in a complement of $\operatorname{Ker} f_{1}$, putting the maximum norm on the summand. Then, the map $g_{1}(x, 0) \equiv g_{1}(x)$ can be written as $g_{1}(x)=f_{1} x_{2}-$ $Q k_{1}\left(x_{1}+x_{2}\right) \oplus-(I-Q) k_{1}\left(x_{1}+x_{2}\right)$. Since a zero for the first summand gives $x_{2}=o\left(\left\|x_{1}\right\|\right)$, by taking the radius $r_{1}$ of $B_{1}$ small enough, the homotopy $f_{1} x_{2}-$ $(1-t) Q k_{1}\left(x_{1}+x_{2}\right) \oplus-(I-Q) k_{1}\left(x_{1}+(1-t) x_{2}\right)$ is admissible (no zeros near the boundary and the condensing condition is verified). Hence, the map $\left(g_{1}(x), \lambda_{M}\right)$ is 0 -epi on $\bar{B}_{1} \times\left(\bar{B}_{2} \cap \Lambda_{M}\right)$ if and only if the map $\left(f_{1} x_{2},(I-Q) k_{1}\left(x_{1}\right), \lambda_{M}\right)$ is 0 -epi on the same set and, since $f_{1}$ is an isomorphism from the complement of Ker $f_{1}$ onto Range $f_{1}$, if and only if the stabilized homotopy class of $(I-Q) k_{1}\left(x_{1}\right)$ is 
nontrivial. If $\operatorname{Ker} f_{1}=\{0\}$, then $\left(f_{1} x, \lambda_{M}\right)$ is always 0 -epi. Note that, from the construction, the map $\left(f-(1-\tau) g_{0}, \lambda_{M}\right)$ is also 0 -epi for $0 \leqslant \tau<1$. Q.E.D.

REMARK 4.2. Note first that if the norm in $F$ is given by the maximum of the norms on Range $f_{1}$ and on the complement of it, then $\|Q\|=1$ and $Q k_{1}$ is condensing if and only if $k_{1}$ is condensing. The assumption on $(I-Q) k_{1}\left(x_{1}+x_{2}\right)$ enables us to perform the deformation to $\left(f_{1} x_{2},(I-Q) k_{1}\left(x_{1}\right)\right)$. Any other similar assumption may replace the previous one: for example, if, close to zero, one has

$$
\left\|k_{1}(x)-k_{1}(y)\right\| \leqslant c(\max \{\|x\|,\|y\|\})^{\beta}\|x-y\|
$$

with $\beta>0$, and $\left\|(I-Q) k_{1}\left(x_{1}\right)\right\| \geqslant C\|x\|^{\alpha}$ with $\alpha<2 \beta+1$ and $k_{1}(0)=0$, then $k_{1}$ is a local contraction (hence $Q k_{1}$ is a $k$-set-contraction); the first part of the equation is uniquely solvable for $x_{2}$ in terms of $x_{1}$ with $\left\|x_{2}\right\| \leqslant K\left\|x_{1}\right\|^{\beta+1}$. Then we have $\left\|(I-Q) k_{1}\left(x_{1}+x_{2}\right)\right\| \geqslant C\left\|x_{1}\right\|^{\alpha}-C^{\prime}\left\|x_{1}\right\|^{2 \beta+1}$.

ReMark 4.3. In Proposition 4.3 we have chosen $B_{1}$ small. Of course, similar results could be obtained for arbitrary $B_{1}$, provided the deformation is valid. For example with conditions on $k_{1}$ of the Landesman-Lazer-Nirenberg type for large $B_{1}$.

REMARK 4.4. It may happen that $(I-Q) k_{1}\left(x_{1}\right) \neq 0$ only on a subspace of $\operatorname{Ker} f_{1}$. Then, by decomposing $\operatorname{Ker} f_{1}$ one may regard the complement of the above subspace as a space of parameters, thus adding its dimension to the local dimension, provided the corresponding stable homotopy class is nontrivial. This argument applies, of course, to the setting of the preceding remark.

REMARK 4.5. It would be extremely interesting to find an example of a mapping $g_{1}(x, \lambda)$ such that $g_{1}(x, 0)$ gives a nontrivial map but such that $\left(g_{1}(x, 0), \lambda\right)$ is trivial (for $\lambda \in \mathbf{R}$ for example) and such that the zeros of $g_{1}(x, \lambda)$ do not satisfy the conclusion of Theorem 4.1. This is equivalent to the following homotopy problem: Let $f: B \times[-1,1] \rightarrow \mathbf{R}^{m}\left(B\right.$ a ball in $\mathbf{R}^{n}$ ) be such that $f(x, t) \neq 0$ on $(\partial B) \times[-1,1]$. Assume that $f(\cdot, 0): \partial B \rightarrow \mathbf{R}^{m} \backslash\{0\}$ defines a nontrivial element in $\Pi_{n-1}\left(S^{m-1}\right)$ (hence by homotopy $f(\cdot, t)$ is nontrivial for each $t$ and so $f^{-1}(0) \cap\left\{t=t_{0}\right\} \neq \varnothing$ ). Assume that $(f(x, t), t)$ as a mapping from $\partial(B \times[-1,1])$ into $\mathbf{R}^{m+1} \backslash\{0\}$ defines a trivial element, the suspension of the preceding, in $\Pi_{n}\left(S^{m}\right)$. Is it then true that $f^{-1}(0)$ has a 1 -dimensional connected subset connecting $B \times\{-1\}$ to $B \times\{1\}$ ? Clearly, this is true if one is in the stable range (the suspension is then an isomorphism).

The last result of this section is not stated in its maximal generality so as to achieve a clearer geometrical meaning (see also Remark 4.6 below).

Proposition 4.4 (ON THE EXISTENCE OF LOOPS AND BUBbLeS). Let $\Lambda$ be the ( possibly infinite dimensional) parameter space and assume that $E=F$. Let $g_{1}(x, \lambda)$ be of the form $x-k_{1}(x, \lambda)$, where $k_{1}$ is compact on $\bar{U}$ and $k_{1}(x, 0)=o(\|x\|)$ close to zero. Suppose that in a neighborhood of $(0,0)$, say of the form $B_{1} \times B_{2}$, the zeros of $g_{1}$ are of the form $(\lambda, x(\lambda))$, with $x(\lambda)$ continuous and $x(0)=0$. Let $\mathscr{C}$ be the connected component of $g_{1}^{-1}(0)$ containing those zeros and assume that $\mathscr{C}$ is bounded and contained in $U$. Then:

(1) There is a bounded open set $V \subset U$ such that $\mathscr{C} \subset V$ and $g_{1}(x, \lambda) \neq 0$ on $\partial V$ and $V$ "looks like" $B_{1} \times B_{2}$ in a neighborhood of zero. 
(2) The set $\mathscr{C} \backslash\{(0,0)\}$ has a closed minimal subset $\Sigma(\bar{\Sigma}=\Sigma \cup\{(0,0)\}$ is the "bubble") such that

(a) $\Sigma$ is connected and has local dimension at least $\operatorname{dim} \Lambda$ and $\Sigma$ has the properties listed in Theorem 3.1 (in particular $\Sigma$ meets $\{\lambda=0\}$ outside $B_{1} \times B_{2}$ );

(b) $\lambda$ is 0 -epi on $\Sigma \cap(V \backslash\{(0,0)\})$, in particular, for any neighborhood $W$ of $(0,0)$, the map $\lambda$ restricted to $\Sigma \cap \partial W$ has no extensions (as those of Definition 2.3) to $\Sigma \cap(U \backslash W)$;

(c) the set $\Sigma \cup\{(0,0)\}$ coincides with the set of zeros of the form $(\lambda, x(\lambda))$ in $a$ neighborhood of $(0,0)$;

(d) each intersection of $\Sigma$ with a hyperplane of the form $E \times\left\{t \lambda_{0}\right\}$, for $\lambda_{0} \in \Lambda$, $\left\|\lambda_{0}\right\|=1$, has a minimal subset $\Sigma_{\lambda_{0}}$ of local dimension at least 1 such that $\Sigma_{\lambda_{0}} \backslash\left\{t \lambda_{0}, x\left(t \lambda_{0}\right),-\varepsilon \leqslant t \leqslant \varepsilon\right\}$ is connected for $\varepsilon$ small enough and there is a map from $\Sigma_{\lambda_{0}} \cup\{(0,0)\}$ into $S^{1}$ which is not homotopic to a constant (i.e., the set $\Sigma_{\lambda_{0}} \cup\{(0,0)\}$ "looks like" a loop $)$.

PROOF. The construction of the set $V$ is by now standard in Nonlinear Functional Analysis and has been used extensively in bifurcation theory (a standard reference is [W]) (see also [I, II, p. 191]), since $\mathscr{C}$ is compact.

Due to the fact that the deformation $\left(x-t k_{1}(x, \lambda), \lambda\right)$ is admissible in any neighborhood of $(0,0)$ contained in $B_{1} \times B_{2}$, the index of the pair at $(0,0)$ is 1 . However, the Leray-Schauder degree of the pair $\left(g_{1}, \lambda\right)$ in $V$ is zero. In fact, if this is not so, then $\lambda$ is 0 -epi on $g_{1}^{-1}(0) \cap \bar{V}$ and, taking into account that $V$ is bounded, one has that $g_{1}^{-1}(0) \cap \partial V \neq \varnothing$ (see Proposition 2.4). Then, by the additivity property of the Leray-Schauder degree, the degree of the pair with respect to $V \backslash \bar{W}$ is 1 for any open neighborhood $W$ of $(0,0)$ contained in $B_{1} \times B_{2}$. Since $V \backslash \bar{W}$ contains the zeros of the pair, when restricted to $V \backslash\{(0,0)\}$, then the degree of the pair, with respect to any open set $V_{1}$ satisfying $g_{1}^{-1}(0) \cap\{\lambda=0\} \subset V_{1} \subset \bar{V}_{1} \subset V \backslash\{(0,0)\}$, is 1. This implies that the pair is 0 -epi on $V \backslash\{(0,0)\}$ and, by Property 2.9, the map $\lambda$ is 0 -epi on $g_{1}^{-1}(0) \cap(V \backslash\{(0,0)\})$. From the compactness of $g_{1}^{-1}(0) \cap V$ the remaining conditions of Theorem 3.1 are met and one gets the subset $\Sigma$ that meets $\{\lambda=0\}$ outside $B_{1} \times B_{2}$ and also meets $\partial W$ for any $W$ as above: this implies (recall that $\Sigma$ is connected) that $\Sigma$ is a subset of $\mathscr{C} \backslash\{(0,0)\}$ and $\bar{\Sigma}=\Sigma \cup\{(0,0)\}$ since $\bar{\Sigma} \cap \partial U=\varnothing$. Decompose now the parameter space $\Lambda$ as $\left\{t \lambda_{0}\right\} \oplus \tilde{\Lambda}, \lambda=t \lambda_{0} \oplus \tilde{\lambda}$. From Property 2.9 the map $t \lambda_{0}$ is 0 -epi on $\{\tilde{\lambda}=0\} \cap \Sigma \cap(V \backslash\{(0,0)\})$. In particular, $t \lambda_{0}$ must change sign on the set $\{\tilde{\lambda}=0\} \cap \Sigma \cap \partial\left\{(x, \lambda):\|x\|<\varepsilon^{\prime},\|\lambda\|<\varepsilon\right\}$ (from the compactness of $\Sigma \cup\{(0,0)\}$, then $\lambda$ is 0 -epi on $\bar{U} \backslash\left\{(x, \lambda):\|x\|<\varepsilon^{\prime},\|\lambda\|<\varepsilon\right\}$ by Proposition 2.3). Clearly, one may choose $\varepsilon^{\prime}, \varepsilon$ so small that on the boundary of the ball $\left\{(x, \lambda):\|x\|<\varepsilon^{\prime},\|\lambda\|<\varepsilon\right\}$ the elements of $\Sigma \cap\{\tilde{\lambda}=0\}$ are of the form $( \pm \varepsilon$, $x( \pm \varepsilon)$ ). This implies (c).

The first part of (d) is again an application of Theorem 3.1. Let $h: \bar{V} \cap\{\tilde{\lambda}=0\}$ $\rightarrow S^{1}$ be the map defined by

$$
h(x, t)= \begin{cases}e^{i \pi t / \varepsilon} & \text { if } x \in \bar{B}_{2} \text { and }|t| \leqslant \varepsilon, \\ -1 & \text { otherwise }\end{cases}
$$

(keep in mind the construction of the set $V$ ). If $\left.h\right|_{\bar{\Sigma}_{\lambda_{0}}}$ is homotopic to a constant, then, by the homotopy extension property (see Remark 2.9), the map $h$ is homotopic 
to a constant also on $\bar{V} \cap\{\tilde{\lambda}=0\}$. Then, there is a lifting [Sp, p. 103] $h_{1}$ : $\bar{V} \cap\{\tilde{\lambda}=0\} \rightarrow \mathbf{R}$ of $h$, which coincides [Sp, p. 54] with the map $t \mapsto \pi t / \varepsilon$ in $\{|t| \leqslant \varepsilon\} \times \bar{B}_{2}$ and such that $h_{1}(x, t) \neq 0$ for $|t| \geqslant \varepsilon$ (from the exponential map). That is the map $\left(g_{1}\left(x, t \lambda_{0}\right), h_{1}(x, t)\right)$ is nonzero on $\partial V$ and on $V \backslash\left(\{|t| \leqslant \varepsilon\} \times \bar{B}_{2}\right)$ which leads to a contradiction since, on one hand, the degree of the pair on $V$ must be the degree on $\{|t| \leqslant \varepsilon\} \times B_{2}$, thus equal to 1 , and, on the other hand ( $g_{1} \neq 0$ on $\partial V)$, the degree of this pair must be 0 . Q.E.D.

REMARK 4.6. In the case when $\Lambda=\mathbf{R}$ one may give a third proof of the existence of the loop (besides that of the exponential map given in part (d) of the above proposition). Let $\mathscr{C}_{ \pm}$be the two pieces of $\mathscr{C}$ emanating from $( \pm \varepsilon, x( \pm \varepsilon))$. If these two pieces do not intersect, then, since they are compact, one may construct two disjoint open sets $V_{ \pm}$with $\mathscr{C}_{ \pm} \subset V_{ \pm}$and $V=V_{+} \cup V_{-} \cup\left(\{|\lambda|<\varepsilon\} \times B_{2}\right)$. Defining $g(x, \lambda)$ as $\lambda$ on $\{|\lambda|<\varepsilon\} \times B_{2}$ and $\pm \varepsilon$ on $V_{ \pm}$, then the degree of $\left(g_{1}, g\right)$ on $V$ is the degree of $\left(g_{1}, \lambda\right)$ on $\{|\lambda|<\varepsilon\} \times B_{2}$, that is 1 , which is impossible since $V$ is bounded and $g_{1}^{-1}(0) \cap \partial V=\varnothing$.

REMARK 4.7. It is clear that the same arguments apply for condensing maps and, also, when the identity is replaced by a Fredholm operator with the degree of $(I-Q) k_{1}\left(x_{1}\right)$ nonzero. It would be interesting to have the same result in the case when the additivity property of the degree is not available.

C. Application to bifurcation. Let $U$ be an open subset of $\Lambda \times E$, where $\Lambda$ and $E$ are Banach spaces. Assume $\Lambda=\tilde{\Lambda} \times \tilde{\tilde{\Lambda}}$, with $\operatorname{dim} \tilde{\Lambda}<+\infty$. The space $\tilde{\tilde{\Lambda}}$ may reduce to $\{0\}$ or may be infinite dimensional, in which case $\tilde{\tilde{\Lambda}}$ is equipped with the family of all finite dimensional subspaces $\tilde{\tilde{\Lambda}}_{M}$ containing a fixed subspace $\tilde{\Lambda}_{M_{0}}$. Assume that $0 \in \bar{B}_{1} \times \bar{B}_{2} \subset U$ and that $g_{1}: U \rightarrow F$ is of the form $g_{1}(\lambda, x)=f_{1}(x)$ $-k_{1}(\lambda, x)$, with $k_{1}(\lambda, 0)=0, f_{1}(t x)=t^{n} f_{1}(x)$, for $t>0$ and for some $n>0$.

Assume that:

(1) $f_{1}$ is proper on $\bar{B}_{2}$.

(2) If $C$ is a bounded subset of $U_{M}=U \cap\left(\tilde{\Lambda} \times \tilde{\Lambda}_{M} \times E\right)$, then $\alpha\left(k_{1}(C)\right) \leqslant$ $K_{M}(C) \alpha\left(f_{1}(C)\right)$, with $K_{M}(C)<K \leqslant 1$ for large $M$ (unless $k_{1}$ is a compact map, $f_{1}$ and $k_{1}$ send bounded sets into bounded sets).

(3) Assume that there is a continuous map $f: \Lambda \rightarrow \tilde{\Lambda}_{M_{0}}$, sending bounded sets into bounded sets, and positive numbers $r_{1}, r_{2}, \varepsilon$ such that if $g_{1}(\lambda, x)=0$ for $\|x\| \leqslant r_{2}$, $r_{1}-\varepsilon \leqslant\|\lambda\| \leqslant r_{1}+\varepsilon$ and $\lambda$ of the form $(\tilde{\lambda}, \tilde{\tilde{\lambda}}=f(\lambda))$, then $x=0$. Recall that $B_{1}=\left\{\lambda:\|\lambda\|<r_{1}\right\}, B_{2}=\left\{x:\|x\|<r_{2}\right\}$ and denote by $B_{1}^{M}$ the set $\left\{\left(\tilde{\lambda}, \tilde{\tilde{\lambda}}_{M}\right)\right.$ : $\tilde{\tilde{\lambda}}_{M} \in \tilde{\tilde{\Lambda}}_{M}$ with $\left.\|\lambda\|<r_{1}\right\}$. (The set $\tilde{\tilde{\lambda}}=f(\lambda)$ will be specified later on as a "surface" which is transversal to an "eigensurface" going through 0 and having as a tangent plane the hyperplane $\tilde{\tilde{\Lambda}}$.) Note that if $\Lambda$ is finite dimensional, then $\tilde{\tilde{\lambda}}-f(\lambda)$ may be any map from $\Lambda$ into $\tilde{\tilde{\Lambda}}$.

THEOREM 4.2. Let $U$ and $g_{1}$ be as above and assume that the map $\left(g_{1}\left(\tilde{\lambda}, \tilde{\tilde{\lambda}}_{M}, x\right)\right.$, $\left.\tilde{\tilde{\lambda}}_{M}-f\left(\tilde{\lambda}, \tilde{\lambda}_{M}\right),\|x\|-r_{2} / 2\right)$ is 0 -epi on $B_{1}^{M} \times B_{2}$ for $M$ large enough (the map $\left(f_{1}(x)-(1-\tau) k_{1}\left(\tilde{\lambda}, \tilde{\tilde{\lambda}}_{M}, x\right), \tilde{\lambda}_{M}-f\left(\tilde{\lambda}, \tilde{\lambda}_{M}\right),\|x\|-r_{2} / 2\right)$ is 0 -epi on the same set if $K=1$ for $\left.0 \leqslant \tau<\tau_{0}\right)$. Then, if $S=\left\{(\lambda, x) \in U: g_{1}(\lambda, x)=0, x \neq 0\right\}$ we have that $S$ has a minimal closed (in $S)$ subset $\Sigma$ such that the map $(\tilde{\tilde{\lambda}}-f(\lambda),\|x\|-\varphi(\|\lambda\|))$ 
is sectionally 0-epi on the set $\Sigma \cap(U \backslash((\Lambda \times\{0\}) \cap \bar{\Sigma}))$, where $\varphi: \mathbf{R}^{+} \rightarrow \mathbf{R}^{+}$is a nonincreasing function with $\varphi(r)=r_{2} / 2$ for $r \leqslant r_{1}$ and $\varphi(r)=0$ for $r \geqslant r_{1}+\varepsilon$. Moreover, $\Sigma$ has the following properties:

(a) The set $\bar{\Sigma} \cap\{(x, \lambda): \tilde{\lambda}=f(\lambda)\}$ intersects $B_{1}^{\varepsilon} \times\{0\}$ and contains a closed connected subset, having local dimension at least 1 , which is either unbounded, or intersects either $\partial U$ or $\Lambda \times\{0\}$ outside $\bar{B}_{1} \times\{0\}$.

(b) If $\Sigma=\Sigma_{1} \cup \Sigma_{2}$, with $\Sigma_{1}, \Sigma_{2}$ two proper closed subsets of $\Sigma$, then $\operatorname{dim}\left(\Sigma_{1} \cap \Sigma_{2}\right)$ $\geqslant \operatorname{dim} \tilde{\Lambda}$. In particular, $\Sigma$ is connected and $\bar{\Sigma}$ has dimension at each point at least $\operatorname{dim} \tilde{\tilde{\Lambda}}+1$ (for points of $\bar{\Sigma} \cap \partial U$ and $\bar{\Sigma} \cap(\Lambda \times\{0\})$ this has to be understood in the sense of Theorem 3.1).

(c) If the estimate of (3) holds for a family of mappings $f_{t}(\lambda)$, then $\Sigma$ is also minimal for the family of corresponding mappings with the same properties.

Proof. Let $A=\left(\Lambda \backslash B_{1}^{\varepsilon}\right) \times\{0\}$. As in the proof of Theorem $4.1 f_{1}$ is proper on closed and bounded subsets of $E$ and $g_{1}$ is proper on closed (in $E$ ) bounded subsets of $U_{M}$, so that the intersection of $\bar{S}$ with bounded and closed (in $E$ ) subsets of $U_{M}$ is compact. Let $g(\lambda, x) \equiv\left(g_{1}(\lambda, x), \tilde{\tilde{\lambda}}-f(\lambda)\right),\|x\|-\varphi(\|\lambda\|)$ and $g_{M}\left(\tilde{\lambda}, \tilde{\tilde{\lambda}}{ }_{M}, x\right)$ be the same map when restricted to $\tilde{\Lambda} \times \tilde{\Lambda}_{M}$. Then the zeros of $g$, which are the zeros of $g_{M}$ for $M \geqslant M_{0}$ by the construction of $f(\lambda)$, are the zeros of $g_{1}$ in $B_{1}^{\varepsilon} \times B_{2}^{\varepsilon}$ with $\tilde{\tilde{\lambda}}=f(\lambda),\|x\|=r_{2} / 2,\|\lambda\|<r_{1}-\varepsilon$, together with the set of $\lambda$ 's with $\tilde{\tilde{\lambda}}=f(\lambda)$, $\|\lambda\|>r_{1}+\varepsilon, x=0$. Furthermore, the zeros of $(\tilde{\lambda}-f(\lambda)),\|x\|-\varphi(\|\lambda\|)$ satisfy the hypotheses of Corollary 4.1 (here the map $f_{2}$ that appears in Corollary 4.1 is given by $f_{2}(\lambda, x)=(\tilde{\lambda},\|x\|)$ which is homogeneous of degree 1 and $f(\lambda)$ is compact). From the hypotheses and Corollary 4.1 it follows that $g_{M}$ is 0 -epi on $U_{M} \backslash\left(\Lambda_{M} \times\{0\}\right)$. By Property 2.9 the map $\left(\tilde{\tilde{\lambda}}{ }_{M}-f\left(\tilde{\lambda}, \tilde{\lambda}_{M}\right),\|x\|-\varphi\left(\left\|\left(\tilde{\lambda}, \tilde{\tilde{\lambda}}_{M}\right)\right\|\right)\right)$ is 0 -epi on $(S \cap(\tilde{\Lambda} \times$ $\left.\left.\tilde{\tilde{\Lambda}}_{M}\right)\right) \cap\left(U_{M} \backslash(\Lambda \times\{0\})\right)$ and, as in the proof of Theorem 4.1, on $\left(S \cap\left(\tilde{\Lambda} \times \tilde{\tilde{\Lambda}}_{M}\right)\right)$ $\cap(U \backslash(\Lambda \times\{0\}))$. Thus, since the complementing map is $g_{N}(\lambda, x)=\lambda_{N}$, then $(\tilde{\tilde{\lambda}}-f(\lambda),\|x\|-\varphi(\|\lambda\|))$ is sectionally 0 -epi on $S \cap(U \backslash(\Lambda \times\{0\}))$. Hence, from Corollary 4.3 and Theorem 3.1, one gets all the properties (b) and (c) of the theorem ( $f_{t}$ being finite dimensional gives an admissible homotopy for sectionally 0 -epi maps).

Finally, by Property 2.9, the map $\|x\|-\varphi(\|\lambda\|)$ is 0 -epi on $\Sigma \cap\{(x, \lambda): \tilde{\tilde{\lambda}}=f(\lambda)\}$. Therefore, by Theorem 3.1, one gets a minimal subset $\Sigma_{1}$ of this set which is connected with local dimension at least 1 and such that $\|x\|-\varphi(\|\lambda\|)$ is 0 -epi on $\Sigma_{1} \cap(U \backslash((\Lambda \times\{0\}) \cap \bar{\Sigma}))$. Moreover, $\Sigma_{1}$ meets the level $\|x\|=r_{2} / 2$ inside $B_{1}^{\varepsilon} \times B_{2}^{\varepsilon}$ and $\Sigma_{1}$ is either unbounded, or $\bar{\Sigma}_{1}$ intersects $\partial U$ or $(\Lambda \times\{0\}) \cap \bar{\Sigma}$. It remains to show that $\bar{\Sigma}_{1}$ intersects $B_{1}^{\varepsilon} \times\{0\}$ and that, if $\Sigma_{1}$ is bounded and $\bar{\Sigma}_{1} \cap \partial U=\varnothing$, then $\bar{\Sigma}_{1}$ also intersects the set of trivial solutions outside $\bar{B}_{1}$. In fact, if $\bar{\Sigma}_{1} \cap\left(\bar{B}_{1}^{\varepsilon} \times\{0\}\right)$ $=\varnothing$, then $\bar{\Sigma}_{1} \cap\left(\bar{B}_{1}^{\varepsilon} \times \bar{B}_{2}\right)$ is compact and, as such, is at a positive distance $d$ from $\bar{B}_{1}^{\varepsilon} \times\{0\}$. The deformation $\|x\|-t \varphi(\|\lambda\|)$ for $d / r_{2} \leqslant t \leqslant 1$ is valid. But then, on $\bar{\Sigma}_{1} \cap\left(\bar{B}_{1} \times \bar{B}_{2}\right)$ we have $\|x\| \neq d / 2$ and this contradicts Property 2.1 . On the other hand, if $\bar{\Sigma}_{1}$ is bounded and does not intersect neither $\partial U$ nor $\Lambda \times\{0\}$ outside $\bar{B}_{1}$, then, again by compactness, the distance $d$ from $\bar{\Sigma}_{1}$ to $(\Lambda \times\{0\}) \backslash\left(B_{1} \times\{0\}\right)$ is positive. By Property 2.2 the map $\|x\|-\varphi(\|\lambda\|)$ is 0 -epi on $\Sigma_{1} \cap V$, where $V$ is the 
intersection of a bounded open neighborhood of $\bar{\Sigma}_{1}$ in $U$ with the set $\{(\lambda, x):\|x\|$ $\left.>\frac{1}{2} \min \left(d, r_{2} / 2\right)\right\}$. But, on $\Sigma_{1} \cap \partial V$, which is contained in $B_{1} \times B_{2}$, the map $\|x\|-\varphi(\|\lambda\|)$ is negative. From Corollary 3.1 one gets a contradiction (also one may argue as follows: From Property 2.9 the map $\|x\|-\varphi(\|\lambda\|)$ is 0 -epi on $\Sigma_{1} \cap$ $\left(U \backslash\left((\Lambda \times\{0\}) \cap \bar{\Sigma}_{1}\right)\right)$ so that, if $(\Lambda \times\{0\}) \cap \bar{\Sigma}_{1}$ is contained in $\bar{B}_{1}^{\varepsilon}$, the map cannot change sign on $\partial V$ ). Q.E.D.

COROLlaRY 4.6. As in Corollary 4.5 assume that $E$ and $F$ have projectional schemes and that the map $g_{M}(\lambda, x) \equiv\left(g_{1}\left(\tilde{\lambda}, \tilde{\tilde{\lambda}}_{M}, x\right), \tilde{\tilde{\lambda}}_{M}-f\left(\tilde{\lambda}, \tilde{\lambda}_{M}\right),\|x\|-\varphi\left(\left\|\left(\tilde{\lambda}, \tilde{\lambda}_{M}\right)\right\|\right)\right)$ satisfies assumptions (2) and (3) of Remark 2.9. Suppose that:

(1) $g_{1}^{-1}(0) \cap\left(\tilde{\Lambda} \times \tilde{\Lambda}_{M}\right)$ intersected with any bounded and closed (in $\left.E\right)$ subset of $U$ is compact for large $M$ (this is true if $g_{1}$ is continuous on $U_{M}$ ).

(2) If $g_{1}(\lambda, x)=0$ for $\|x\| \leqslant r_{2}, r_{1}-\varepsilon \leqslant\|\lambda\| \leqslant r_{1}+\varepsilon$ and $\lambda$ of the form $(\tilde{\lambda}, \tilde{\lambda}=$ $f(\lambda)$ ), then $x=0$, where $f(\lambda)$ is as in Theorem 4.2.

(3) $g_{M}(\lambda, x)$ is A-proper 0 -epi on $B_{1} \times B_{2}$ for $M \geqslant M_{0}$.

Then, there is a minimal subset $\Sigma$ of $S \equiv g_{1}^{-1}(0) \backslash(\Lambda \times\{0\})$ such that $(\tilde{\bar{\lambda}}-f(\lambda)$, $\|x\|-\varphi(\|\lambda\|))$ is sectionally 0 -epi on $\Sigma \cap(U \backslash((\Lambda \times\{0\}) \cap \bar{\Sigma}))$, with the properties of Theorem 4.2.

Proof. As in the proof of Corollary 4.5 it is enough to show that one has control on the zeros of $g_{n}$ near $\|\lambda\|=r_{1}$ at the level $\|x\|=r_{2} / 2$. If $g_{1, n}\left(\lambda_{n}, x_{n}\right)=0$ for $r_{1}-\varepsilon \leqslant\left\|\lambda_{n}\right\| \leqslant r_{1}, \tilde{\lambda}_{n}=f\left(\lambda_{n}\right)$, one would get, from the $A$-properness of $g$, a subsequence converging to $(\lambda, x)$ with $g(\lambda, x)=0$ and $\|x\|=r_{2} / 2, r_{1}-\varepsilon \leqslant\|\lambda\| \leqslant$ $r_{1}$. The rest of the proof follows from Corollary 4.4(b), modifying the argument for an infinite dimensional parameter space as in the proof of Theorem 4.2. Q.E.D.

In Theorem 4.2 the decomposition of $\Lambda$ has been used only in order to specify the dimension of the space where the range of the map $\tilde{\lambda}-f(\lambda)$ lies.

We shall give below sufficient conditions for the map $g_{M}$ to be 0-epi on $B_{1}^{M} \times B_{2}$ which will be applied to the usual bifurcation situation.

Lemma 4.1. Assume that $g_{1}$ and $U$ satisfy properties (1) and (2) (in the case when $K=1$ assume that, for $C$ contained in $B_{2}, K_{M}(t C) \leqslant \tilde{K}_{M}(C)$ for all $\left.t \in[0,1]\right)$ and that, in a neighborhood of $B_{1} \times B_{2}$, the map $g_{1}(\lambda, x)$ has the form $f_{1}(x)-T(\lambda)(x)-$ $h_{1}(\lambda, x)$, with $T(\lambda)(x)$ homogeneous in $x$ of degree $n\left(\right.$ as $\left.f_{1}\right)$ and $\left\|h_{1}(\lambda, x)\right\|=o\left(\|x\|^{n}\right)$ uniformly for $\lambda$ in $\tilde{\Lambda} \times \tilde{\Lambda}_{M}$. Assume also that the equation $\tilde{\lambda}=f(\lambda)$ is solvable in that neighborhood in the form $\tilde{\tilde{\lambda}}=\tilde{f}(\tilde{\lambda})$, with $\tilde{f}$ continuous. Assume finally that, for $r_{1}-\varepsilon \leqslant\|\lambda\| \leqslant r_{1}+\varepsilon$, the map $f_{1}-T(\tilde{\lambda}, \tilde{f}(\tilde{\lambda}))$ is one-to-one. Then, for $r_{2}$ small enough, the map $g_{M}$ is 0-epi on $B_{1}^{M} \times B_{2}$ if and only if the map $\left(f_{1}(x)-T(\tilde{\lambda}, \tilde{f}(\tilde{\lambda}))(x)\right.$, $\left.\lambda_{M}-f(\lambda),\|x\|-r_{2} / 2\right)$ is 0 -epi on $B_{1}^{M} \times B_{2}$. In the case when $K=1$, replace $k_{1}$ everywhere by $(1-\tau) k_{1}$ with $0<\tau<\tau_{0}$. Then if the map with $(1-\tau) k_{1}$ is 0 -epi, it is so with $\tau=0$.

Proof. Note first that, from the homogeneity of $f_{1}$ and $T(\lambda)$ and from the smallness assumption on $h_{1}$, it follows that for any bounded subset $C$ of $E$ we have $\alpha\left(T\left(\tilde{\lambda}, \tilde{\tilde{\lambda}}_{M}\right)(C)\right) \leqslant K_{M}(C) \alpha\left(f_{1}(C)\right)$ for $\left(\tilde{\lambda}, \tilde{\lambda}_{M}\right)$ in a neighborhood of $B_{1}^{M}$. In fact, if $C$ is such a set, then for $t$ small enough the set $t C$ is contained in $B_{2}$, so that there is 
an $\varepsilon(t)$ such that $\left\|h_{1}\left(\tilde{\lambda}, \tilde{\tilde{\lambda}}_{M}, t x\right)\right\| \leqslant \varepsilon(t) t^{n}$ for $x \in C$ and $\varepsilon(t) \rightarrow 0$ as $t \rightarrow 0$. Then

$$
\begin{aligned}
\alpha\left(\left(T\left(\tilde{\lambda}, \tilde{\tilde{\lambda}}_{M}\right)(\cdot)+h_{1}(\lambda, \cdot)\right)(t C)\right) & =t^{n} \alpha\left(\left(T\left(\tilde{\lambda}, \tilde{\tilde{\lambda}}_{M}\right)(\cdot)+h_{1}(\lambda, t \cdot) / t^{n}\right)(C)\right) \\
& \leqslant K_{M}(t C) t^{n} \alpha\left(f_{1}(C)\right) .
\end{aligned}
$$

Hence $\alpha\left(T\left(\tilde{\lambda}, \tilde{\lambda}_{M}\right)(C)\right) \leqslant \tilde{K}_{M}\left(t_{0} C\right) \alpha\left(f_{1}(C)\right)+2 \varepsilon(t)$ for $t_{0}$ small enough and $t \leqslant t_{0}$. Therefore, the map $T\left(\tilde{\lambda}, \tilde{\bar{\lambda}}_{M}\right)$ is condensing with respect to $f_{1}$ (in the case when $k_{1}$ is $k$-set-contractive with respect to $f_{1}$, so is $T\left(\tilde{\lambda}, \tilde{\lambda}_{M}\right)$ with the same constant $\left.k\right)$. In particular $f_{1}-T\left(\tilde{\lambda}, \tilde{\tilde{\lambda}}_{M}\right)$ is proper on $\bar{B}_{2}$. Then for each $(\tilde{\lambda}, f(\tilde{\lambda}))$ with $\left|\|\lambda\|-r_{1}\right| \leqslant \varepsilon$ there is an $r_{2}$ such that $g_{1}$ satisfies the hypothesis (3) of Theorem 4.2. In fact, if there is a sequence $\left\|x_{K}\right\|$ tending to zero such that $g_{1}\left(x_{K}, \lambda\right)=0$, then setting $y_{K}=$ $x_{K} /\left\|x_{K}\right\|$ and using the homogeneity of $f_{1}$ and $T(\lambda)$ one would have $f_{1}\left(y_{K}\right)$ $T(\lambda)\left(y_{K}\right)=h_{1}\left(\lambda, x_{K}\right) /\left\|x_{K}\right\|^{n}$. Since the right-hand side tends to zero and the left-hand side is proper, one would get a contradiction with the properties of $f_{1}-T(\lambda)$. The continuity of $\tilde{f}(\tilde{\lambda})$ and the compactness of the set $(\tilde{\lambda}, \tilde{f}=\tilde{f}(\tilde{\lambda}))$ (recall that $f$ has range in $\left.\tilde{\Lambda}_{M_{0}}\right)$ when intersected with $\bar{B}_{1}\left(r_{1}+\varepsilon\right)$, will give a uniform lower bound for $r_{2}$ such that the hypothesis is verified. Then let $C$ be a bounded set in $B_{1}^{M} \times B_{2}$. Choose $t_{1}$ such that $\left\|h_{1}(\lambda, t x) t^{-n}\right\| \leqslant(1-K) \alpha\left(f_{1}(C)\right) / 4$ for $\left(\tilde{\lambda}, \tilde{\tilde{\lambda}}_{M}, x\right)$ in $C$ and $0 \leqslant t \leqslant t_{1}$ (if $K=1$, then $K$ is replaced by $1-\tau, 0<\tau<\tau_{0}$ ). Then,

$$
\begin{aligned}
\alpha\left(T(\cdot)+h_{1}(\cdot, \cdot)(\cdot)^{-n}\right)\left(C \times\left[0, t_{1}\right]\right) & \leqslant \alpha(T(\cdot)(C))+(1-K) \alpha\left(f_{1}(C)\right) / 2 \\
& \leqslant(1+K) \alpha\left(f_{1}(C)\right) / 2
\end{aligned}
$$

( $k_{1}$ replaced by $(1-\tau) k_{1}$ if $K=1$ ). Dividing the interval $\left[t_{1}, 1\right]$ in small slices, the argument of Proposition 4.1 together with the fact that $(1+K) / 2<1$ implies that the homotopy $f_{1}(x)-T(\lambda)(x)-h_{1}(\lambda, t x) t^{-n}$ is admissible (from the homogeneity it follows that the zeros are not moved for $t>0$ ). One may then replace in $g_{M}$ the term $g_{1}(\lambda, x)$ by $f_{1}(x)-T\left(\tilde{\lambda}, \tilde{\tilde{\lambda}}_{M}\right)(x)$. The finite dimensional deformation $T\left(\tilde{\lambda},(1-t) \tilde{\lambda}_{M}+t \tilde{f}(\tilde{\lambda})\right)$ is also admissible. Finally, since $k_{1}$ and $T(\lambda)$ are condensing with respect to $f_{1}$, from Corollaries 2.2 and 2.4 one gets the last statement of the lemma. Q.E.D.

In the classical bifurcation theory $f_{1}-T(\lambda)$ is a linear operator, corresponding to the linearization of $g_{1}(\lambda, x)$ near $\lambda=0$, so that $n=1$ and $T(0)=0$. As noted in the proof of Proposition 4.3, the fact that $f_{1}$ is proper implies in this case that $f_{1}$ is semi-Fredholm. In the rest of the paper $f_{1} \equiv A$ will stand for a Fredholm operator.

Lemma 4.2. Assume that $f_{1} \equiv A$ is a linear Fredholm operator with $\operatorname{dim} \operatorname{Ker} A=d$, codim Range $A=d^{*}$ and that $T(\lambda)$ is a family of linear operators, continuous in $\lambda$ in the norm topology, with $T(0)=0$. Then there is an $r_{1}$ small enough such that, if $\|\lambda\| \leqslant r_{1}, A-T(\lambda)$ is a Fredholm operator of index $d-d^{*}$ and the spectral properties of $A-T(\lambda)$ are given by the spectral properties of a $d \times d^{*}$ matrix $B(\lambda)$ with $B(0)=0$. In particular, if $d=d^{*}$, the points $\lambda$ for which $A-T(\lambda)$ is not an isomorphism are given by the zeros of $\operatorname{det} B(\lambda)$.

Proof. This result is proved in [I,I, pp. 43, 44 and 48]. For the reader's convenience we shall give the form of $B(\lambda)$. (In $[\mathbf{I}, \mathbf{I}]$ the map $T(\lambda)$ was assumed to 
be real analytic in $\lambda$. For the above result this is not necessary.) Let $Q$ be a projection from $F$ into Range $A$, decompose $E$ as $\operatorname{Ker} A \oplus E_{2}, x=x_{1}+x_{2}$ and let $K$ : Range $A \rightarrow E_{2}$ be the inverse of $A$. Then $A x-T(\lambda) x$ can be written as

$$
A x_{2}-Q T(\lambda)\left(x_{1}+x_{2}\right) \oplus-(I-Q) T(\lambda)\left(x_{1}+x_{2}\right)
$$

and also as

$$
\begin{aligned}
(A-Q T(\lambda))\left[x_{2}-(I-K Q T(\lambda))^{-1} K Q T(\lambda) x_{1}\right] \\
\quad \oplus-(I-Q) T(\lambda)(I-K Q T(\lambda))^{-1} x_{1} \\
\quad-(I-Q) T(\lambda)\left[x_{2}-(I-K Q T(\lambda))^{-1} K Q T(\lambda) x_{1}\right],
\end{aligned}
$$

where $r_{1}$ has been chosen such that $\|K Q T(\lambda)\|<1$, hence $A-Q T(\lambda)$ is an isomorphism for all $\lambda \in \tilde{\Lambda} \times \tilde{\Lambda}_{M}$. This implies that all the spectral information is given by $B(\lambda) \equiv-(I-Q) T(\lambda)(I-K Q T(\lambda))^{-1}$ restricted to Ker $A$. Q.E.D.

Even if $A$ has index 0 and $T(\lambda)$ is real analytic in $\lambda$, the set of zeros of $\operatorname{det} B(\lambda)$ may be very complicated, being the union of algebraic varieties of different dimensions (if $\lambda$ is complex and $T(\lambda)$ is complex analytic, then this set is an algebraic variety of complex codimension 1 ) (see [I,I, Chapter II]).

In order to apply the sufficient condition of Lemma 4.1 and hence Theorem 4.1 to our next result, it is necessary for $A$ to have nonpositive index $(A-T(\lambda)$ one-to-one for $\|\lambda\| \simeq r_{1}$, on the "surface" $\left.\tilde{\lambda}=\tilde{f}(\tilde{\lambda})\right)$ and, in order to get a nontrivial invariant, for $A$ to have index 0 (the case of nonzero index will be considered later). Since one is working in a neighborhood of $\lambda=0$, then $r_{1}$ is thought of as arbitrarily small, so that the "surface" $\tilde{\lambda}=\tilde{f}(\tilde{\lambda})$ is transversal to one of the "eigensurfaces" going through 0 and parametrized by $\tilde{\lambda}$. The reason for the form of the "transversal surface" is that, if its dimension does not complement the dimension of the eigensurface, then as explained in $[\mathbf{I}, \mathbf{I}$ and $\mathbf{I}, \mathbf{I I}]$, the local invariant would be automatically 0 . Hypotheses of the form $\tilde{\tilde{\lambda}}=L \tilde{\lambda}+\tilde{\tilde{\lambda}}_{0}, L$ a linear map, $\tilde{\lambda}_{0}$ fixed of small norm, are examples of such transversal surfaces.

Proposition 4.5. Assume that $A$ is a Fredholm operator of index 0 and that $T(\lambda)$ is as above. Assume that one has a continuous family of functions $f_{t}(\lambda)$ such that for $\|\lambda\| \leqslant r_{1}$ the zeros of $\tilde{\bar{\lambda}}=f_{t}(\lambda)$ have the form $\tilde{\tilde{\lambda}}=\tilde{f}_{t}(\tilde{\lambda})$ and for $\|\lambda\|=r_{1}$, the operator $A-T\left(\tilde{\lambda}, \tilde{f}_{t}(\tilde{\lambda})\right)$ is invertible. Assume that $f_{0}(\lambda)=0$ for $\|\lambda\| \leqslant r_{1}$. Then $g_{M}$ as defined in Theorem 4.2 is 0 -epi on $B_{1}^{M} \times B_{2}$ if and only if the stable image in $\Pi_{K+d-1}\left(S^{d}\right)$ under the Whitehead's J-homomorphism, of the class of $B(\tilde{\lambda}, 0)$ in $\Pi_{K-1}(\mathrm{GL}(d))$, is nontrivial. Here $K=\operatorname{dim} \tilde{\Lambda}, d=\operatorname{dim} \operatorname{Ker} A$. (For more details on the J-homomorphism see [I,II, Chapter I].) (If $\operatorname{dim} E$ and $\operatorname{dim} \Lambda$ are finite, then this corresponds to the $\operatorname{dim} E_{2}+\operatorname{dim} \tilde{\tilde{\Lambda}}$ suspension.)

Proof. From the continuity of $\tilde{f}_{t}$ and Lemma 4.1 one gets an $r_{2}$ such that hypothesis (3) is valid for the family $f_{t}$ and $B\left(\tilde{\lambda}, \tilde{f}_{t}(\tilde{\lambda})\right)$ is invertible for $\left\|\left(\tilde{\lambda}, \tilde{f}_{t}(\tilde{\lambda})\right)\right\|$ $=r_{1}$ and by continuity also for an interval $\left[r_{1}-\varepsilon, r_{1}+\varepsilon\right]$. Thus, from Lemmas 4.1 and 4.2, one has to look at the map $\left(A x-T\left(\tilde{\lambda}, \tilde{f}_{t}(\tilde{\lambda})\right) x, \tilde{\lambda}_{M}-f_{t}(\lambda),\|x\|-r_{2} / 2\right)$. Decomposing $A-T(\lambda)$ into two summands, as in Lemma 4.2, one may perform the 
following compact perturbations. In the first summand put

$$
x_{2}-(1-\tau)(I-K Q T(\lambda))^{-1} K Q T(\lambda) x_{1}
$$

and in the second summand put

$$
(1-\tau)\left(x_{2}-(1-\tau)(I-K Q T(\lambda))^{-1} K Q T(\lambda) x_{1}\right):
$$

for $\|\lambda\|=r_{1}, \lambda=\left(\tilde{\lambda}, \tilde{f}_{t}(\tilde{\lambda})\right), A-T(\lambda)$ is invertible so that those deformations are admissible. Then $g_{M}$ is 0 -epi if and only if $\left((A-Q T(\lambda)) x_{2}, B(\lambda) x_{1}, \tilde{\tilde{\lambda}}_{M}-f_{t}(\lambda)\right.$, $\left.\|x\|-r_{2} / 2\right)$ is. Since $T(\lambda)$ is small in norm, hence strictly contracting on $E_{2}$ with respect to $A$, one may use the deformation $A-Q T(\tau \lambda)$, replace $B(\lambda)$ by $B\left(\tilde{\lambda}, \tilde{f}_{t}(\tilde{\lambda})\right)$ and, sending $t$ to 0 , the map $g_{M}$ is 0 -epi if and only if $\left(A x_{2}, B(\tilde{\lambda}, 0) x_{1}, \tilde{\tilde{\lambda}}_{M}\right.$, $\left.\|x\|-r_{2} / 2\right)$ is. That is if and only if the stable class of $\left(B(\tilde{\lambda}, 0) x_{1},\|x\|-r_{2} / 2\right)$ in $\Pi_{K+d-1}\left(S^{d}\right)$ is nontrivial $\left(\left(A x_{2}, \tilde{\tilde{\lambda}}_{M}\right)\right.$ acts as a suspension $)$ and this is just the construction of the Whitehead map (see [I,II]). Q.E.D.

REMARK 4.8. If the index of $A$ is positive and $\operatorname{Ker} A$ splits into $E_{1} \oplus W$ with $\operatorname{dim} E_{1}=d^{*}$ such that $B(\tilde{\lambda}, \tilde{f}(\tilde{\lambda}))$ restricted to $E_{1}$ and $\|\lambda\|=r_{1}$ is invertible, then $W$ may be considered as a new space of parameters and one may replace $B(\lambda)$ by a $d \times d$ matrix $\tilde{B}(\lambda)$ by adding $d-d^{*}$ equations (this splitting is always possible if $T(\lambda)$ is real analytic; see [I,I, pp. 29 and 47]). If $\tilde{B}(\tilde{\lambda}, 0)$ is written as $\left(B_{1}(\tilde{\lambda}), B_{W}(\tilde{\lambda})\right)$ with $B_{1}(\tilde{\lambda})$ a $d^{*} \times d^{*}$ matrix which is invertible for $\|\tilde{\lambda}\|=r_{1}, B_{W}(\tilde{\lambda})$ a $d^{*} \times$ $\left(d-d^{*}\right)$ matrix, then one may extend $B(\lambda)$ to the matrix

$$
\left(\begin{array}{cc}
B_{1}(\tilde{\lambda}) & B_{W}(\tilde{\lambda}) \\
0 & C(\tilde{\lambda})
\end{array}\right)
$$

with $C(0)=0, C(\tilde{\lambda})$ invertible for $\|\tilde{\lambda}\|=r_{1}$. This new matrix has the homotopy class in $\Pi_{K-1}(\mathrm{GL}(d))$ of

$$
\left(\begin{array}{cc}
B_{1}(\tilde{\lambda}) & 0 \\
0 & C(\tilde{\lambda})
\end{array}\right)
$$

so that if $B_{1}$ has a nontrivial stable class one may choose $C(\tilde{\lambda})$ to be $\|\tilde{\lambda}\| I$, or if $B_{1}$ has a trivial class (hence deformable to $I$ ) and if $K \leqslant d-d^{*}$, then one may choose $C(\lambda)$ so that this matrix has nontrivial stable class if $K \equiv 0,1,2,4[8]$ and such that its image under the $J$-homomorphism is nontrivial. In fact, from the exact sequence

$$
\Pi_{K}\left(S^{d-d^{*}}\right) \rightarrow \Pi_{K-1}\left(\mathrm{GL}\left(d-d^{*}\right)\right) \stackrel{i_{*}}{\rightarrow} \Pi_{K-1}\left(\mathrm{GL}\left(d-d^{*}+1\right)\right) \rightarrow \Pi_{K-1}\left(S^{d-d^{*}}\right)
$$

it follows that for $K \leqslant d-d^{*}$, the map $i_{*}$ is onto, hence under the Bott periodicity theorem and the Adams results on the $J$-homomorphism, one may always choose $C(\lambda)$ to be a generator of those groups with nontrivial $J$-image. In particular, if $K=1$ one always gets a connected set $\Sigma$ with local dimension at least $d-d^{*}+1$ (see [I,I, p. 47] for a local version of this fact) if the index is positive. If $K=2$ and $d-d^{*} \geqslant 2$ this is also the case: in $[\mathbf{I}, \mathbf{I}]$ one had this local result for $\lambda \in \mathbf{C}$ and $A$ a complex operator of positive complex index $\tilde{d}-\tilde{d}^{*}\left(2\left(d-d^{*}\right)\right.$ as a real operator). For a negative index one could trade off the negative index against some subspace of the parameter space (see [I,I, p. 61]). 
REMARK 4.9. It is not necessary to linearize $g_{1}$ or to take $r_{1}$ small in order to obtain sufficient conditions. For example if $E=F, f_{1}(x)=x$, then by using the weak homotopy equivalence between parametrized maps defined on $S^{K-1}$, of compact and condensing type (see [AF and Bo]), one may restrict oneself to compact $k_{1}$ and, using the decomposition of the Fredholm operator $A$ (with nonnegative index) to the case when $A=I, I-k_{1}(\cdot, \lambda)$ maps $E$ into $E^{\infty-n}$ a fixed $n$-codimensional space of $E$. Then $g_{M}$ will be 0 -epi on $B_{1}^{M} \times B_{2}$ if and only if its stable homotopy class (as an element of $\Pi^{\infty-n-K+1}\left(\partial\left(B_{1}^{M} \times B_{2}\right)\right)$, see [GG]) is nontrivial.

In our next proposition we shall give a necessary and sufficient condition for that to be true when $\operatorname{dim} \tilde{\Lambda}=1$, which includes the cases studied in [AA,I; AA,II; FMP,I and FMP,II] (where $E^{\infty-n}=E$ ).

Proposition 4.6. Let $g_{1}$ be of the form $g_{1}(\lambda, x)=x-k_{1}(\lambda, x)$ from $E \times \Lambda$ into $E^{\infty-n}$, a n-codimensional subspace of $E$, and where $k_{1}$ is compact on finite dimensional subspaces of $\Lambda$ and $k_{1}(\lambda, 0) \equiv 0$. Assume that there are two points $\lambda_{1}, \lambda_{2}$ and a positive number $\varepsilon$ such that:

(1) If $g_{1}(\lambda, x)=0$ for $\|x\| \leqslant 2 \varepsilon,\left\|\lambda-\lambda_{i}\right\| \leqslant \varepsilon, i=1,2$, and $\lambda$ is on the line joining $\lambda_{1}$ with $\lambda_{2}$, then $x=0$.

(2) The generalized index of $g_{1}\left(\lambda_{1}, x\right)$ is different from the generalized index of $g_{1}\left(\lambda_{2}, x\right)$ (as elements of $\Pi^{\infty-n}(S), S$ a sphere in $E$; if $n=0$, then these are the usual Leray-Schauder indices).

Then, the set of nontrivial solutions has a minimal closed subset $\Sigma$ with the properties of Theorem 4.2. In particular $\Sigma$ is connected, has dimension at each point at least $\operatorname{dim} \Lambda$. Moreover, $\bar{\Sigma} \cap\left\{(\lambda, x): \lambda\right.$ belongs to the line going through $\lambda_{1}$ and $\left.\lambda_{2}\right\}$ intersects that line in the plane $x=0$ strictly on the segment between $\lambda_{1}$ and $\lambda_{2}$ and, if bounded, meets again that line strictly outside that segment.

Proof. Without loss of generality one may assume that the line going through $\lambda_{1}$ and $\lambda_{2}$ is represented by a one-dimensional subspace $\tilde{\Lambda}$ and that $\lambda_{1}=\left(-r_{1}, 0\right)$, $\lambda_{2}=\left(r_{1}, 0\right)$, where $0 \in \tilde{\Lambda}$. Taking $B_{1}=\left\{\|\lambda\|<r_{1}+\varepsilon\right\}, B_{2}=\{\|x\|<2 \varepsilon\}, f(\lambda) \equiv 0$, the three hypotheses of Theorem 4.2 are satisfied. It remains to show that $\left(g_{1}\left(\tilde{\lambda}, \tilde{\tilde{\lambda}}_{M}, x\right), \tilde{\tilde{\lambda}}_{M},\|x\|-\varepsilon\right)$ is 0-epi on $B_{1}{ }^{M} \times B_{2}$. First, as above, deform, $\tilde{\tilde{\lambda}}_{M}$ to 0 in $x-k_{1}\left(\tilde{\lambda}, \tilde{\lambda}_{M}, x\right)\left(k_{1}\right.$ is compact on $\left.\mathbf{R} \times \tilde{\Lambda}_{M} \times E\right)$. Then use the following homotopy on the last term: $(1-t)(\|x\|-\varepsilon)+t\left(r_{1}-|\tilde{\lambda}|\right)$ which is positive if $\|x\|>\varepsilon$ and $|\tilde{\lambda}|<r_{1}$ while, if $\|x\| \leqslant 2 \varepsilon$ and $r_{1} \leqslant|\tilde{\lambda}| \leqslant r_{1}+\varepsilon$, a zero of $g_{1}(\tilde{\lambda}, 0, x)$ must have $x=0$. Hence the last term is negative so that the homotopy has its zeros in the set $\left\{|\lambda| \leqslant r_{1}\right\} \times\{\|x\| \leqslant \varepsilon\}$ and $g_{M}$ is deformable on $B_{1}^{M} \times B_{2}$ to

$$
\left(g_{1}(\tilde{\lambda}, 0, x), \tilde{\tilde{\lambda}}_{M}, r_{1}-|\tilde{\lambda}|\right) \equiv F_{M}(\lambda, x) \text {. }
$$

Then let

$$
\Omega=B_{1}^{M} \times B_{2} \backslash\left(\bar{B}_{+1}^{M} \times \bar{B}_{2}^{\prime} \cup \bar{B}_{-1}^{M} \times \bar{B}_{2}^{\prime}\right),
$$

where $B_{ \pm 1}^{M}=\left\{\left(\lambda=\left(\tilde{\lambda}, \tilde{\tilde{\lambda}}_{M}\right):\left\|\lambda \mp r_{1}\right\|<\varepsilon / 2\right)\right\}$ and $B_{2}^{\prime}=\{\|x\|<\varepsilon\}$. Then, $F_{M}$ sends $\bar{\Omega}$ into $\mathbf{R} \times \mathbf{R}^{M} \times E^{\infty-n} \backslash\{0\}$, and $\left.F_{M}\right|_{\partial\left(B_{1}^{M} \times B_{2}\right)},\left.F_{M}\right|_{\partial\left(B_{+1}^{M} \times B_{2}^{\prime}\right)}$ have stable homotopy classes in $\Pi^{\infty-n}\left(S^{1}\right)$, where $S^{1}$ is a generic sphere in $\mathbf{R} \times \mathbf{R}^{M} \times E$, so that the sum of the last two classes is equal to the first (the generalized cohomotopy degree, 
as defined in [I,II, pp. 160 and 190]) of $F_{M}$ with respect to $\partial \Omega$ is 0 , since $F_{M}$ is nonzero on $\Omega$. Finally, the class of $F_{M}$ on $\partial\left(B_{-1}^{M} \times B_{2}^{\prime}\right)$ is the $(M+1)$-suspension of the class of $g_{1}\left(-r_{1}, 0, x\right)$. Hence the class of $F_{M}$ in $\Pi^{\infty-n}\left(\partial\left(B_{1}^{M} \times B_{2}\right)\right)$ is nontrivial if and only if these two $(M+1)$-suspensions are different, i.e., if and only if $g_{M}$ is 0 -epi on $B_{1}^{M} \times B_{2}$ (for the case $n=0$ see [I,I, p. 79]). Note that if $\operatorname{dim} E=d<+\infty$, then $g_{M}$ on $\partial\left(B_{1}^{M} \times B_{2}\right)$ defines an element of $\Pi_{M+d}\left(S^{M+d-n}\right)$ and $g\left( \pm r_{1}, 0, x\right)$ elements of $\Pi_{d-1}\left(S^{d-n-1}\right)$. If $\operatorname{dim} E=+\infty$, then the stable classes have to be different. Q.E.D.

COROllary 4.7. The conclusions of Proposition 4.6 remain valid if the line going through $\lambda_{1}$ and $\lambda_{2}$ is replaced by a curve defined in the form $\tilde{\lambda}-f(\lambda)=0$ with $f$ having range in $\tilde{\Lambda}_{M_{0}}$, i.e., the curve is given by the zeros of a mapping from $\Lambda$ into $\tilde{\Lambda}$, a 1-codimensional subspace of $\Lambda$, such that $\lambda_{1}=\left(-r_{1}, 0\right), \lambda_{2}=\left(r_{1}, 0\right)$ and one of the following conditions is satisfied:

(a) The curve, in the balls $\left\|\lambda-\lambda_{i}\right\| \leqslant \varepsilon, i=1,2$, can be parametrized by $\tilde{\lambda}$ and, furthermore, the curve between $\lambda_{1}$ and $\lambda_{2}$ is contained in a box of the form $|\tilde{\lambda}|<r_{1}$, $\left|\tilde{\bar{\lambda}}_{M}\right|<R$ and the curve outside the portion between $\lambda_{1}-\varepsilon$ and $\lambda_{2}+\varepsilon$ does not enter the box $|\tilde{\lambda}| \leqslant r_{1}+\varepsilon,|\tilde{\tilde{\lambda}} M| \leqslant R+\varepsilon$. The map $\varphi$ of Theorem 4.2 is $\varepsilon$ inside the first box and 0 outside the second box.

(b) $g_{1}$ is defined only on an open subset $U$ of $\Lambda \times \underset{\tilde{\Lambda}}{E}$ and there is a mapping $\psi$ : $\Lambda \rightarrow \Lambda$ such that $\left.\psi\right|_{\tilde{\Lambda}_{N_{0}}}=I\left(\tilde{\Lambda}_{N_{0}}\right.$ a complement in $\tilde{\Lambda}$ of $\left.\tilde{\Lambda}_{M_{0}}\right)$ such that $\psi$ is a homeomorphism and the image of the curve under $\psi$ satisfies the conditions of (a) (for example $\psi=$ identity if $U$ contains the boxes of (a)) in which case one has a third possibility for $\bar{\Sigma}$, namely that it may meet $\partial U$. The map $\varphi$ is of the form $\varphi^{\prime}\left(\psi^{-1}\right)$, with $\varphi^{\prime}$ as in (a) above.

(c) $g_{1}$ is defined on $U$ and $\psi$ is a homeomorphism only in a neighborhood of the portion of the curve between $\lambda_{1}$ and $\lambda_{2}$ : in this case one has the conclusion of (b) for $U$, if $n=0$ (classical degree theory), or in general for that neighborhood. This is the case if 0 is a regular value of $\tilde{\lambda}_{M}-f(\lambda) . \varphi$ is $\varepsilon$ on the curve between $\lambda_{1}$ and $\lambda_{2}$ and vanishes outside the above neighborhood.

Proof. Note first that the scaling property is true if one replaces $B_{1}$ by any convex set containing the origin, in particular for the boxes of (a). Now, from the hypotheses of (a) we may perform in the box $|\tilde{\lambda}| \leqslant r_{1}+\varepsilon / 2,\|\tilde{\tilde{\lambda}} M\| \leqslant R,\|x\| \leqslant 2 \varepsilon$, the deformations $g_{1}\left(\tilde{\lambda},(1-t) \tilde{\tilde{\lambda}}_{M}+t f(\lambda), x\right)$ and then

$$
(1-t)\left(\|x\|-\varphi(\|\lambda\|)+t\left(r_{1}-|\tilde{\lambda}|\right)\right)
$$

(from the hypotheses of (a)). We get to the point where the sum of the classes of $\left(g_{1}(\tilde{\lambda}, f(\lambda), x), \tilde{\tilde{\lambda}}_{M}-f(\lambda), r_{1}-|\tilde{\lambda}|\right)$ on $\bar{B}_{ \pm 1}^{M} \times \bar{B}_{2}^{\prime}$ has to be zero. Then, if on $B_{+1}$ say, this curve is given by $\tilde{\tilde{\lambda}}_{M}=\tilde{f}(\tilde{\lambda})$ with $\tilde{f}\left(r_{1}\right)=0$, one may replace, in successive order, $(\tilde{\tilde{\lambda}}, f(\lambda))$ in $g_{1}$ by $\left(r_{1}, 0\right)$ (linear deformation), $\tilde{\tilde{\lambda}}_{M}-f(\lambda)$ by $\tilde{\tilde{\lambda}}_{M}-$ $f\left(\tilde{\lambda}, \quad(1-t) \tilde{\tilde{\lambda}}_{M}+t \tilde{f}(\tilde{\lambda})\right)$ and, finally, $\lambda_{M}-f(\tilde{\lambda}, \tilde{f}(\tilde{\lambda}))=\tilde{\lambda}_{M}-\tilde{f}(\tilde{\lambda})$ by $\tilde{\tilde{\lambda}}_{M}-$ $(1-t) \tilde{f}(\tilde{\lambda})$ since $\tilde{f}\left(r_{1}\right)=0$.

For (b) it is enough to note that $g_{M}(\lambda, x)$ is 0 -epi (on $U$ for example) if and only if $g_{M}(\psi(\lambda), x)$ is 0 -epi (on $\psi(U)$ ) and that $\psi$ induces an isomorphism at the 
cohomotopy level (see [I,I Chapter III]). Hence the difference of the classes in $\Lambda \times E$ goes into the difference of the classes in $\psi(\Lambda) \times E$ (this is the extension of the classical composition rule for degree).

For (c), in the case $n=0$, it is enough to recall that the scaling property was introduced as a substitute for the excision property. The last statement is proved as in [FMP,II, proof of Theorem 2.4]. Q.E.D.

REMARK 4.10. Note that if $g_{1}$ has a linearization at $\left(\lambda_{1}, 0\right)$, in general one may not ask for $x=0$ to be an isolated zero if the linearization maps $E$ into $E^{\infty-n}$ with $n>0$, unless one may replace globally $g_{1}$ by a mapping from $E$ into $E$ of zero index as in Remark 4.9.

REMARK 4.11. In the classical bifurcation situation, one supposes that one has a good knowledge of the behavior of $g_{1}(\lambda, x)$ near $x=0$, that is, that $g_{1}$ is linearizable. As it has been seen in Proposition 4.5, the spectral properties of the linearization are usually given in terms of a finite dimensional matrix, in particular, if $n=0$, by the zeros of det $B(\lambda)$. Now, generically these zeros form a surface of codimension 1 , so that the situation of Proposition 4.6 is important for this case (more details will be given below). However, there are other generic situations where the zeros of det $B(\lambda)$ are not of codimension 1: for example, if $\Lambda$ and $E$ are complex Banach spaces with $T(\lambda)$ analytic in $\lambda$, then the sets of zeros of $\operatorname{det} B(\lambda)$ have (real) codimension 2 and the local indices do not change (there is always a path joining $\lambda_{1}$ with $\lambda_{2}$ and not meeting the zeros of $\operatorname{det} B(\lambda)$ ) so that Proposition 4.6 does not apply (see [I,I, Chapter I]) since one has to consider all the spaces as real. In fact, if the linearization has the form $I-T(\lambda)$ with $T(\lambda)$ compact and analytic in $\lambda$, then the set of points $\lambda$ where $I-T(\lambda)$ is not invertible has a discrete intersection with any complex plane $\tilde{\Lambda}$, provided that this plane contains one point $\lambda_{0}$ where $I-T\left(\lambda_{0}\right)$ is invertible (see [I,I, p. 45]). If $\lambda_{1}$ is a singular point for $I-T(\lambda)$ and det $B(\lambda)=a\left(\lambda-\lambda_{1}\right)^{n}+\cdots$ for the corresponding $B(\lambda)$ and $\lambda$ in that complex plane, then if $n$ is odd, the map $\left(g_{1}(\lambda, x), \tilde{\lambda}_{M},\|x\|-r_{2} / 2\right)$ is 0 -epi on $B_{1}^{M} \times B_{2}$ and one has the subset $\Sigma$ of the nontrivial solutions branching off $\left(\lambda_{1}, 0,0\right)$ and of local dimension at least $\operatorname{dim} \Lambda-1(\operatorname{dim} \Lambda$ is the dimension over the reals) with the properties of Theorem 4.2. This follows from Proposition 4.5 and, clearly, the hyperplane $\tilde{\tilde{\Lambda}}$ may be replaced by any analytic surface $\tilde{\Lambda}_{M}-f(\lambda)$. Note also that, in the same context, if $n$ is even, then one may construct a nonlinear part $h_{1}(\lambda, x)$ such that $\left(\lambda_{1}, 0,0\right)$ is not a bifurcation point for $g_{1}(\lambda, x)$ (the proof of this fact will be given in a subsequent paper). It would be very interesting to construct an example where $\Sigma$ has local dimension exactly $\operatorname{dim} \Lambda-1$. This seems to be difficult since once one is on the bifurcated branch one has to avoid the continuation situation and, furthermore, if $\operatorname{dim} \operatorname{ker}\left(I-T\left(\lambda_{1}\right)\right)=1$ one has bifurcation in all directions of a complex plane (see [I,I, p. 45]).

REMARK 4.12. Another instance where the set of singular points has codimension 2 is for the problem of periodic solutions of autonomous differential equations: $\dot{x}=f(\lambda, x), \lambda \in \Lambda$. No classical degree theory may be applied and one has to use either an invariant in $\Pi^{\infty-1}(S)$, as in [I,I] , or, using the equivariant character of the problem, in $\Pi^{\infty}\left(\mathbf{C} P^{\infty}\right)\left(\mathbf{C} P^{\infty}\right.$ a complex projective space) as in [I,III]. The equivariance will give that the local dimension is in fact at least $\operatorname{dim} \Lambda+1$. (The method of 
the present paper gives only $\operatorname{dim} \Lambda$. The result for this case is included in a paper in preparation where the equivariant problem is considered in its generality.)

Our last results concern the structure of the set of bifurcation points $\Sigma_{0} \equiv \bar{\Sigma} \cap(\Lambda$ $\times\{0\})$. Denote by $U_{0}$ the set $U \cap(\Lambda \times\{0\})$ and assume $\operatorname{dim} \tilde{\Lambda} \geqslant 1$.

Proposition 4.7. Let $\Sigma, f(\lambda)$ and $\varphi(\lambda)$ be as in Theorem 4.2 and assume that $(\tilde{\tilde{\lambda}}-f(\lambda),\|x\|-\varphi(\lambda))$ is sectionally 0 -epi on $\Sigma \cap\left(U-\Sigma_{0}\right)$. Then $\tilde{\tilde{\lambda}}-f(\lambda)$ is 0-epi on $\Sigma_{0} \cap\left(U_{0}-A_{0}\right)$, where $A_{0} \equiv\left\{\lambda \in \Lambda: \tilde{\lambda}=f(\lambda),\|\lambda\| \geqslant r_{1}\right\}$. In particular $\Sigma_{0}$ has a minimal closed (in $\left.U_{0}-A_{0}\right)$ subset $\Sigma_{1}$ such that $\tilde{\tilde{\lambda}}-f(\lambda)$ is 0 -epi on $\Sigma_{1} \cap\left(U_{0}-\right.$ $\left.\bar{\Sigma}_{1} \cap A_{0}\right)$. $\Sigma_{1}$ meets the zeros of $\tilde{\lambda}-f(\lambda)$ inside $B_{1}, \bar{\Sigma}_{1}$ is either unbounded or meets $\partial U_{0} \cup A_{0} . \Sigma_{1}$ is connected and $\bar{\Sigma}_{1}$ has dimension at each point at least $\operatorname{dim} \tilde{\Lambda}$.

Proof. Since the zeros of $(\tilde{\bar{\lambda}}-f(\lambda),\|x\|-\varphi(\lambda))$ on $\Sigma$ are located in $D \equiv$ $B\left(r_{1}-\varepsilon\right) \times\left\{x:\|x\|=r_{2} / 2\right\}$, then, from Property 2.2, this map is sectionally 0-epi on $\Sigma \cap\left(U-\left(\Sigma_{0} \cup\left(A_{0} \times E\right)\right)\right)$. Furthermore on this set one may perform the homotopy $\|x\|-(1-t) \varphi(\lambda)-t r_{2} / 2$, since the zeros of the pair are either in $A_{0} \times E$ or in $D$. Similarly $(\tilde{\tilde{\lambda}}-f(\lambda),\|x\|-\eta)$ is sectionally 0-epi on $\Sigma \cap\left(U-\left(\Sigma_{0}\right.\right.$ $\left.\left.\cup\left(A_{0} \times E\right)\right)\right)$ for any $0<\eta<r_{2}$.

Suppose now that $\tilde{\tilde{\lambda}}-f(\lambda)$ is not sectionally 0 -epi on $\Sigma_{0} \cap\left(U_{0}-A_{0}\right)$. Then there is a finite dimensional bounded map $h(\lambda)$ with bounded support contained in $U_{0}-A_{0}$, such that $\tilde{\lambda}-f(\lambda)-h(\lambda) \neq 0$ on $\Sigma_{0}$. Since $f(\lambda)+h(\lambda)$ is finite dimensional (in some $\tilde{\tilde{\Lambda}}_{M}$ ) and compact, there exists an open neighborhood $V$ of $\Sigma_{0} \cap\left(\operatorname{Supp} h \cup \bar{B}_{1}\right)$ such that $\tilde{\tilde{\lambda}}-f(\lambda)-h(\lambda) \neq 0$ on $V$. Furthermore it is clear that for $\eta$ small enough, if $(\lambda, x)$ belongs to $\bar{\Sigma}$ with $\lambda$ in $\left(\tilde{\Lambda} \times \tilde{\Lambda}_{M}\right) \cap\left(\operatorname{Supp} h \cup \bar{B}_{1}\right)$ and $\|x\| \leqslant 2 \eta$, then $\lambda$ is in $V$. Also, from the fact that the compact set $\left(\tilde{\Lambda} \times \tilde{\Lambda}_{M}\right) \cap$ Supp $h$ is contained in $U_{0}$, one may choose $\eta$ smaller such that $((\tilde{\Lambda} \times \tilde{\Lambda}) \cap \operatorname{Supp} h)$ $\times\{x:\|x\| \leqslant 3 \eta\}$ is contained in $U$. Then take $\psi(x)$ a real function with value 0 for $\|x\| \leqslant \eta / 2$ or $\|x\| \geqslant 2 \eta$ and value 1 for $\|x\|=\eta$. Let $\varphi(\lambda, x)$ be a Urysohn's function with values 0 on a neighborhood of $\partial U$ and 1 on $\left(\left(\tilde{\Lambda} \times \tilde{\Lambda}_{M}\right) \cap(\operatorname{Supp} h \cup\right.$ $\left.\left.\bar{B}_{1}\right)\right) \times\{x:\|x\| \leqslant 2 \eta\}$. The map $\psi(x) h(\lambda) \varphi(\lambda, x)$ has bounded support contained in $U-\left(\Sigma_{0} \cup\left(A_{0} \times E\right)\right)$, thus $(\tilde{\tilde{\lambda}}-f(\lambda)-\psi(x) h(\lambda) \varphi(\lambda, x),\|x\|-\eta)$ must have a zero in $\Sigma \cap\left(U-\left(\Sigma_{0} \cup\left(A_{0} \times E\right)\right)\right)$. For this point, $\psi(x)=1$ and either $\lambda$ is in $\left(\tilde{\Lambda} \times \tilde{\Lambda}_{M}\right) \cap \operatorname{Supp} h$ so that $\varphi(\lambda, x)=1$, or $h(\lambda)=0$ and $\lambda$ belongs to $B_{1}$. Hence one has a zero of $(\tilde{\lambda}-f(\lambda)-h(\lambda),\|x\|-\eta)$ on $\Sigma$ and $\lambda$ must belong to $V$, giving a contradiction.

Finally, since $\tilde{\Lambda}$ is finite dimensional and $f$ is compact, $\tilde{\tilde{\lambda}}-f(\lambda)$ is proper on bounded and closed subsets of $\Lambda$ thus, from Property $2.10 \tilde{\tilde{\lambda}}-f(\lambda)$ is 0 -epi on $\Sigma_{0} \cap\left(U_{0}-A_{0}\right)$. The rest of the proof follows, as in Corollary 4.2, from Theorem 3.1 and Property 2.8. Q.E.D.

In the case when $\operatorname{dim} \tilde{\Lambda}=1$, one may give more information on $\Sigma_{0}$. For simplicity we shall assume, in our last proposition, that $U_{0}$ is convex.

LEMmA 4.3. Let $U_{0}$ be an open subset of $\Lambda, \Sigma_{0}$ be closed and $\lambda_{1}=\left(-r_{1}, 0\right)$, $\lambda_{2}=\left(r_{1}, 0\right)$ be two points of $U_{0}-\Sigma_{0}$. Then:

(1) The following properties are equivalent:

(a) $\Sigma_{0}$ disconnects $U_{0}$ between $\lambda_{1}$ and $\lambda_{2}$, i.e. any path in $U_{0}$ from $\lambda_{1}$ to $\lambda_{2}$ meets $\Sigma_{0}$;

(b) $\left(\tilde{\tilde{\lambda}}, \tilde{\lambda}^{2}-r_{1}^{2}\right)$ is 0 -epi on $U_{0}-\Sigma_{0}$; 
(c) $\left(\tilde{\tilde{\lambda}}, \tilde{\lambda}^{2}-r_{1}^{2}\right)$ is not homotopic on $U_{0}-\Sigma_{0}$ to $(\tilde{\tilde{\lambda}}, c)$, c a positive constant, via a finite dimensional homotopy, $((\tilde{\lambda}, c)$ is trivial $)$.

(2) If $U_{0}$ is star-shaped with respect to $\lambda_{2}$ and $\Sigma_{0}$ disconnects $U_{0}$ between $\lambda_{1}$ and $\lambda_{2}$, then $\Sigma_{0}$ has a closed minimal subset $\tilde{\Sigma}$ which disconnects $U_{0}$ (i.e. no proper closed subset of $\tilde{\Sigma}$ has this property). If $\tilde{\Sigma}=\Sigma_{1} \cup \Sigma_{2}$ with $\Sigma_{1}, \Sigma_{2}$ two proper closed subsets of $\tilde{\Sigma}$, then $\operatorname{dim} \Sigma_{1} \cap \Sigma_{2} \geqslant \operatorname{dim} \Lambda-2$. In particular $\tilde{\Sigma}$ is connected and has local dimension at least $\operatorname{dim} \Lambda-1$. (If $U_{0}$ is homeomorphic to a finite dimensional sphere this result is contained in [K, vol. II, p. 471].)

Proof. (1) Suppose first that there is a path $\lambda(t)$ in $U_{0}, t$ in $[0,1], \lambda(0)=\lambda_{1}$, $\lambda(1)=\lambda_{2}$, which does not intersect $\Sigma_{0}$. From the compactness of the path one may assume that it is at a distance larger than some $\varepsilon$ from $\bar{\Sigma}$ and, by taking a piecewise linear approximation, that it lies in some $\tilde{\Lambda} \times \tilde{\Lambda}_{M}$. One may modify the path so that, close to $\lambda_{1}$, it coincides with the segment joining $\lambda_{1}$ to $\left(-r_{1}+\varepsilon, 0\right)$ and, close to $\lambda_{2}$, with the segment joining $\left(r_{1}-\varepsilon, 0\right)$ to $\lambda_{2}$. Finally one may move the path so that whenever it crosses the hyperplane $\tilde{\lambda}=r_{1}$ it does so traversally (hence a finite even number of crossings) and such that, if $t_{0}$ corresponds to a crossing, then $\lambda\left(t_{0}+\varepsilon \tau\right)$ $=\left(r_{1}+\varepsilon \tau, \mu_{0}, \nu_{0}\right)$ for $\tau$ in $[-1,1], \mu_{0} \neq 0$ and $\left(\mu_{0}, \nu_{0}\right)$ depending only on $t_{0}$. Consider now the following finite dimensional homotopy:

$$
\left(\tilde{\tilde{\lambda}}_{N},\left(-2 r_{1}\right)^{-1}\left(\left(\tilde{\lambda}(t)-r_{1}\right) \tilde{\tilde{\lambda}}_{M}-\left(\tilde{\lambda}-r_{1}\right) \tilde{\tilde{\lambda}}(t)\right),(\tilde{\lambda}-\tilde{\lambda}(t))\left(\tilde{\lambda}-r_{1}\right)\right)
$$

for $0 \leqslant t \leqslant t_{0}-\varepsilon, t_{0}$ corresponding to the first crossing. The zeros of the second component represent the line joining $\lambda_{2}$ to $\lambda(t)$ and the zeros of the homotopy are at $\lambda_{2}$ and $\lambda(t)$. At $t_{0}-\varepsilon$ perform the deformation

$$
\begin{aligned}
&\left(\tilde{\lambda}_{N},\left(-2 r_{1}\right)^{-1}\right.\left(-\varepsilon \mu-\left(\tilde{\lambda}-r_{1}\right) \mu_{0}\right), \\
&\left(-2 r_{1}\right)^{-1}\left(-\varepsilon \nu-\left((1-t)\left(\tilde{\lambda}-r_{1}\right)-t \varepsilon \mu \mu_{0}^{-1}\right) \nu_{0}\right), \\
&\left.(1-t)\left(\tilde{\lambda}-r_{1}+\varepsilon\right)\left(\tilde{\lambda}-r_{1}\right)+t \mu\left(\mu-\mu_{0}\right)\right),
\end{aligned}
$$

where $\tilde{\tilde{\lambda}}_{M} \equiv(\mu, \nu)$. This homotopy does not move the line from $\lambda_{2}$ to $\lambda\left(t_{0}-\varepsilon\right)$, nor its zeros, but reparametrizes the line with $\mu$ instead of $\tilde{\lambda}$. Next cross the hyperplane $\tilde{\lambda}=r_{1}$ with the homotopy

$$
\left(\tilde{\tilde{\lambda}}_{N},\left(-2 r_{1}\right)^{-1}\left(\varepsilon \tau \mu-\left(\tilde{\lambda}-r_{1}\right) \mu_{0}\right),\left(2 r_{1}\right)^{-1}\left(\varepsilon \nu-\varepsilon \mu \mu_{0}^{-1} \nu_{0}\right), \mu\left(\mu-\mu_{0}\right)\right)
$$

for $\tau$ in $[-1,1]$. Finally return to the parametrization by $\tilde{\lambda}$ for the line joining $\lambda_{2}$ to $\lambda\left(t_{0}+\varepsilon\right)$, via the homotopy

$$
\begin{array}{r}
\left(\tilde{\tilde{\lambda}}_{N},\left(-2 r_{1}\right)^{-1}\left(\varepsilon \mu-\left(\tilde{\lambda}-r_{1}\right) \mu_{0}\right),\left(2 r_{1}\right)^{-1}\left(\varepsilon \nu-\left((1-t) \varepsilon \mu \mu_{0}^{-1}+t\left(\tilde{\lambda}-r_{1}\right)\right) \nu_{0}\right),\right. \\
\left.(1-t) \mu\left(\mu-\mu_{0}\right)+t\left(\tilde{\lambda}-r_{1}-\varepsilon\right)\left(\tilde{\lambda}-r_{1}\right)\right) .
\end{array}
$$

One may then follow the homotopy $\left(\tilde{\tilde{\lambda}}_{N},\left(-2 r_{1}\right)^{-1}\left(\left(\tilde{\lambda}(t)-r_{1}\right) \tilde{\tilde{\lambda}}_{M}^{\prime}-\left(\tilde{\lambda}-r_{1}\right) \tilde{\tilde{\lambda}}^{\prime}(t)\right)\right.$, $\left.(\tilde{\lambda}-\tilde{\lambda}(t))\left(\tilde{\lambda}-r_{1}\right)\right)$ until the next crossing, where $\tilde{\tilde{\lambda}}^{\prime}$ is $(\mu,-\nu)$ if $\tilde{\lambda}$ is $(\mu, \nu)$. Since there is an even number of crossings, one gets for $t=1-\varepsilon$ the map

$$
\left(\tilde{\tilde{\lambda}}_{N},\left(2 r_{1}\right)^{-1} \varepsilon \tilde{\tilde{\lambda}}_{M},\left(\tilde{\lambda}-r_{1}+\varepsilon\right)\left(\tilde{\lambda}-r_{1}\right)\right)
$$

which is clearly homotopic to $\left(\tilde{\tilde{\lambda}}_{N}, \tilde{\tilde{\lambda}}_{M},\left(\tilde{\lambda}-r_{1}\right)^{2}\right)$ and to $(\tilde{\tilde{\lambda}}, c)$ on $U_{0}-\Sigma_{0}$ for a positive $c$. Noting that, from Corollary $2.1,\left(\tilde{\bar{\lambda}}, \tilde{\lambda}^{2}-r_{1}^{2}\right)$ is not 0 -epi on $U_{0}-\Sigma_{0}$ one 
has proved the implications $b \Rightarrow c \Rightarrow a$. Finally if $\Sigma_{0}$ disconnects $U_{0}$, the map $\left(\tilde{\lambda}, \tilde{\lambda}^{2}-r_{1}^{2}\right)$ has local indices -1 at $\lambda_{1}$ and 1 at $\lambda_{2}$ (hence Leray-Schauder degree 0 on $U_{0}$ ) but is nevertheless 0 -epi on $U_{0}-\Sigma_{0}$ : given any bounded open $V$, with $V \subset \bar{V} \subset U_{0}-\Sigma_{0}$ and containing $\lambda_{1}$ and $\lambda_{2}$, these points belong to two different components of $V$ on which the map has a nonzero degree (see Remark 2.7). This finishes the proof of part (1).

For (2), let $\mathscr{C}$ be the family of closed subsets $C$ of $\Sigma_{0}$ such that any path from $\lambda_{1}$ to $\lambda_{2}$ cuts $C$. If $\mathscr{C}^{\prime}$ is a chain in $\mathscr{C}$, let $\tilde{\Sigma}$ be the intersection of all elements of $\mathscr{C}^{\prime}$. From the compactness of the paths, $\tilde{\Sigma}$ cuts any path (and therefore is nonempty). By Zorn's lemma $\mathscr{C}$ has a minimal element, also denoted by $\tilde{\Sigma}$. Now, if $\tilde{\Sigma}=\Sigma_{1} \cup \Sigma_{2}$, then, from the minimality of $\tilde{\Sigma}$, there are paths $\lambda_{1}(t)$ and $\lambda_{2}(t)$ joining $\lambda_{1}$ to $\lambda_{2}$ and avoiding $\Sigma_{1}$ and $\Sigma_{2}$. These paths may be chosen as before. In particular, if $\operatorname{dim} \Lambda=\infty$ and $\operatorname{dim} \Sigma_{1} \cap \Sigma_{2} \leqslant n<\infty$, one may choose $\tilde{\Lambda}_{M}$ such that $\operatorname{dim} \tilde{\Lambda}_{M} \geqslant$ $n+3$.

Let $K$ be the compact set of all line segments $\left[\lambda_{2}, \lambda_{1}(t)\right],\left[\lambda_{2}, \lambda_{2}(t)\right]$. Since $U_{0}$ is star-shaped with respect to $\lambda_{2}$, the distance from $K$ to $\partial U_{0}$ is positive. Choose some positive $\delta$ such that $3 \delta$ is less than that distance and define $V_{i}=\left\{\lambda \in U_{0}\right.$ : distance $(\lambda, K)<i \delta\}$ for $i=1,2,3$. Then $K \subset V_{1} \subset V_{2} \subset \bar{V}_{3} \subset U_{0}$. Let $\varphi_{i}(\lambda)$ be an Urysohn's function with values 1 on $\bar{V}_{i}$ and 0 on $V_{i+1}^{c}$ for $i=1,2$. Now write the homotopies based on $\lambda_{1}(t)$ and $\lambda_{2}(t)$ as $\left(\tilde{\tilde{\lambda}}-h_{i}(\lambda, t), k_{i}(\lambda, t)\right)$ with $h_{i}(\lambda, t)$ in $\tilde{\Lambda}_{M}$, $i=1,2$. By inspection of the homotopy, it is easy to see that on the line $\tilde{\tilde{\lambda}}-h_{i}(\lambda, t)$ $=0$ and outside the segment $\left[\lambda_{2}, \lambda_{i}(t)\right]$, the term $k_{i}(\lambda, t)$ is positive. On $V_{3}-\Sigma_{i}$, $i=1,2$, define the new homotopy

$$
g_{i}(\lambda, t)=\left(\tilde{\tilde{\lambda}}-\varphi_{2}(\lambda) h_{i}(\lambda, t), \varphi_{1}(\lambda) k_{i}(\lambda, t)+\left(1-\varphi_{1}(\lambda)\right) c\right) .
$$

The zeros of $g_{i}(\lambda, t)$ are $\lambda_{2}$ and $\lambda_{i}(t)$. Clearly $g_{1}(\lambda, 0)=g_{2}(\lambda, 0)$ is homotopic on $V_{3}-\tilde{\Sigma}$ to $\left(\tilde{\tilde{\lambda}}, \tilde{\lambda}^{2}-r_{1}^{2}\right)$, via a linear deformation on the last term. $g_{1}(\lambda, 1)=g_{2}(\lambda, 1)$ $=(\tilde{\tilde{\lambda}}, c), g_{i}(\lambda, t)=(\tilde{\tilde{\lambda}}, c)$ when restricted to $\partial V_{3} \times[0,1]$. Note finally that, since $g_{i}(\lambda, t)$ is proper, one may suppose, from Proposition 2.3, that one is working on $\overline{V_{3}-\Sigma_{i}}$ and, from Corollary $2.1 \mathrm{~b}$, it is enough to define the homotopies on $\partial V_{3} \cup \Sigma_{i}$. We shall apply Lemma 3.1 on the sets $B_{1}=B_{2}=\left(\partial V_{3} \times[0,1]\right) \cup(\tilde{\Sigma} \times$ $\{0\}) \cup(\tilde{\Sigma} \times\{1\}), A_{i}=B_{1} \cup\left(\Sigma_{i} \times[0,1]\right), i=1,2, A=A_{1} \cup A_{2}$. The maps $g_{1}$ and $g_{2}$ are those defined above and $g(\lambda, t)$ is $(\tilde{\tilde{\lambda}}, c)$ on $\left(\partial V_{3} \times[0,1]\right) \cup(\tilde{\Sigma} \times\{1\})$, $g_{1}(\lambda, 0)$ on $\tilde{\Sigma} \times\{0\}$ and any finite dimensional extension on $\tilde{\Sigma} \times[0,1]$. If $\operatorname{dim} \Sigma_{1} \cap$ $\Sigma_{2}<\operatorname{dim} \Lambda-2$, then, from [P, p. 181] $\operatorname{dim}\left(\Sigma_{1} \cap \Sigma_{2}\right) \times[0,1]<\operatorname{dim} \Lambda-1$. Since $g_{1}$ and $g_{2}$ differ only on this last set, from Lemma 3.1, $\left.g\right|_{B_{1} \cup B_{2}}$ has a finite dimensional extension from $A$ into $\Lambda-\{0\}$. That is $g(\lambda, 0)$ is homotopic to $g(\lambda, 1)$, via a finite dimensional homotopy and so, from Corollary $2.1 \mathrm{~b}, g(\lambda, 0)$ is not 0 -epi on $\overline{V_{3}-\tilde{\Sigma}}$, hence $\tilde{\Sigma}$ does not separate $V_{3}$ which is clearly a contradiction. The rest of the proof follows as in Theorem 3.1. Note that $\tilde{\Sigma}$ is always connected for a general open set $U_{0}$. If this were not the case, then $\tilde{\Sigma}=\Sigma_{1} \cup \Sigma_{2}$ with $\Sigma_{1} \cap \Sigma_{2}=\varnothing$ and one could choose $\lambda_{1}(t)=\lambda_{2}(t)$. Q.E.D.

Proposition 4.8. Assume that $U_{0}$ is convex, $\operatorname{dim} \tilde{\Lambda}=1, \operatorname{dim} \tilde{\Lambda} \geqslant 1$. Suppose that $(\tilde{\tilde{\lambda}},\|x\|-\varphi(\lambda))$ is sectionally 0 -epi on $\Sigma \cap\left(U_{0}-\Sigma_{0}\right)$, where $\varphi(\lambda)$ is as in Proposition 4.6. Let $\lambda_{1}=\left(-r_{1}, 0\right), \lambda_{2}=\left(r_{1}, 0\right)$. Then:

(1) $\Sigma_{0}$ has a closed connected minimal subset $\tilde{\Sigma}$ of local dimension at least $\operatorname{dim} \tilde{\Lambda}$, 
which disconnects $U_{0}$ between $\lambda_{1}$ and $\lambda_{2}$. Also if $\tilde{\Sigma}=\Sigma_{1} \cup \Sigma_{2}$ with $\Sigma_{1}, \Sigma_{2}$ two proper closed subsets of $\tilde{\Sigma}$, then $\operatorname{dim} \Sigma_{1} \cap \Sigma_{2} \geqslant \operatorname{dim} \Lambda-2\left(\right.$ if $\operatorname{dim} \tilde{\tilde{\Lambda}}=1$, then $\Sigma_{1} \cap \Sigma_{2} \neq$ $\varnothing)$.

(2) For any pair of points $\mu_{1}, \mu_{2}$ with $\mu_{i}$ in the connected component of $\lambda_{i}$ in $U_{0}-\Sigma_{0}$, $i=1,2$, then either $\bar{\Sigma}$ meets $\partial U$ above the segment $\left[\mu_{1}, \mu_{2}\right]$ (this includes the case when $\Sigma$ is unbounded above this segment), or $\Sigma$ covers $\mu_{1}$ or $\mu_{2}$. If the first part of this alternative fails for all pairs $\left(\mu_{1}, \mu_{2}\right)$, for example with a priori bounds, then $\Sigma$ covers at least one of the components, i.e. for any $\mu$ in this component there is an $x$ such that $(\mu, x)$ is in $\Sigma$.

Proof. Part (1) will follow from Lemma 4.3 once we show that $\Sigma_{0}$ disconnects $U_{0}$. If this is not so, there is a path $\lambda(t)$, lying in some $\tilde{\Lambda} \times \tilde{\Lambda}_{M}$ at a distance at least $\varepsilon$ from $\bar{\Sigma}$, transversal to the hyperplane $\tilde{\lambda}=r_{1}$ with $\lambda\left(t_{0}+\varepsilon \tau\right)=\left(r_{1}+\varepsilon \tau, \mu_{0}, \nu_{0}\right)$, $\mu_{0} \neq 0$, close to a crossing at $t_{0}$. Assume that the path, close to $\lambda_{1}$, coincides with the segment from $\lambda_{1}$ to $\left(-r_{1}-\varepsilon, 0\right)$ and, close to $\lambda_{2}$, with the segment from $\lambda_{2}$ to $\left(r_{1}+\varepsilon, 0\right)$. One may also assume that the path does not re-enter the box $\left\{|\tilde{\lambda}| \leqslant r_{1}+\right.$ $\varepsilon\} \times\{\|\tilde{\lambda}\| \leqslant \varepsilon\}$ outside these segments (if it does so, it must cut $\Sigma_{0}$ inside the box, from Corollary 4.7). Note that one has an odd number of crossings of the hyperplane $\tilde{\lambda}=r_{1}$. Finally deform $\varphi(\lambda)$ so that its value $\eta$ is less than $\varepsilon$ above the segment $\left[\lambda_{1}, \lambda_{2}\right]$ and so that $\varphi(\lambda)$ is 0 outside the above box. Consider then the homotopy $\left(\tilde{\lambda}-h(\lambda, t),\|x\|-\varphi_{t}(\lambda)\right)$, where $h(\lambda, t)$ corresponds to the rotation of the line from $\lambda_{2}$ to $\lambda(t)$ around the point $\lambda_{2}$, with its reparametrization near the crossings as in Lemma 4.3, and $\varphi_{t}(\lambda)$ has values $\eta$ on the segment $\left[\lambda_{2}, \lambda(t)\right]$ and 0 outside the box consisting of all $\lambda$ 's at a distance less than $\varepsilon$ from that segment. Since $U_{0}$ is convex and open and the set $K$ of segments is compact, the zeros of the homotopy on $\Sigma$ are above the segment $\left[\lambda_{2}, \lambda(t)\right]$ and at a distance of at least $\varepsilon$ from the endpoints. Then one has that $\left(\tilde{\tilde{\lambda}}_{N}, \mu,-\nu,\|x\|-\varphi_{1-\varepsilon}(\lambda)\right)$ is sectionally 0-epi on $\Sigma \cap\left(U-\Sigma_{0}\right)(-\nu$ comes from the odd number of crossings) and so $\bar{\Sigma}$ must intersect the segment between $\lambda_{2}$ and $\left(r_{1}+\varepsilon, 0\right)$ as in Theorem 4.2 , leading to a contradiction. Note that if $\operatorname{dim} \tilde{\tilde{\Lambda}}=1$, one does not need a reparametrization of the lines. Note also that the map $\left(\tilde{\lambda}_{N}, \mu, \nu,\|x\|-\varphi_{1-\varepsilon}(\lambda)\right)$ would also be sectionally 0 -epi since this notion does not depend on orientation.

For the second part of the proposition, assume there is a pair of points $\mu_{i}=\left(s_{i}, \tilde{\tilde{\lambda}}_{i}\right)$ in the component of $\lambda_{i}, i=1,2$, such that $\bar{\Sigma}$, above the segment $\left[\mu_{1}, \mu_{2}\right]$, is bounded and contained in $U$ and such that $\Sigma \cap\left(\left\{\lambda=\mu_{i}\right\} \times E\right)=\varnothing$. By slightly moving $\lambda_{1}$, $\lambda_{2}$ and the coordinate system in $\Lambda$, one may assume that $s_{1} \neq r_{1}$ and $s_{1} \neq s_{2}$. Construct paths $\lambda_{i}(t)$ from $\lambda_{i}$ to $\mu_{i}$, in the component of $\lambda_{i}$, lying in some $\tilde{\Lambda} \times \tilde{\Lambda}_{M}$ for $i=1,2$ as in the first part. The first path is transversal to the hyperplane $\tilde{\lambda}=r_{1}$ and the second to the hyperplane $\tilde{\lambda}=s_{1}$. Rotating the lines $\left[\lambda_{2}, \lambda_{1}(t)\right]$ around $\lambda_{2}$, the map $\left(\tilde{\tilde{\lambda}}_{N},\left(s_{1}-r_{1}\right) \tilde{\lambda}_{M}-\left(\tilde{\lambda}-r_{1}\right) \tilde{\tilde{\lambda}}_{1},\|x\|-\varphi_{1}(\lambda)\right)$ will be sectionally 0-epi on $\Sigma \cap\left(U-\Sigma_{0}\right)$, where $\varphi_{1}$ has values $\eta$ on the line between $\lambda_{2}$ and $\mu_{1}$ and 0 outside an $\varepsilon$-tubular neighborhood of that line. $(\eta<\varepsilon$ and both chosen as above uniformly on the paths.) Writing the above map as $\left(\tilde{\tilde{\lambda}}_{N},\left(s_{1}-r_{1}\right)\left(\tilde{\tilde{\lambda}}_{M}-\tilde{\tilde{\lambda}}_{1}\right)-\left(\tilde{\lambda}-s_{1}\right) \tilde{\tilde{\lambda}}_{1},\|x\|-\right.$ $\left.\varphi_{1}(\lambda)\right)$ and rotating the segments $\left[\mu_{1}, \lambda_{2}(t)\right]$ around $\mu_{1}$, the map

$$
\left(\tilde{\tilde{\lambda}}_{N},\left(s_{1}-s_{2}\right)\left(\tilde{\tilde{\lambda}}_{M}-\tilde{\lambda}_{1}\right)-\left(\tilde{\lambda}-s_{1}\right)\left(\tilde{\tilde{\lambda}}_{1}-\tilde{\tilde{\lambda}}_{2}\right),\|x\|-\varphi_{2}(\lambda)\right)
$$


will be sectionally 0 -epi on $\Sigma \cap\left(U-\Sigma_{0}\right)$, where $\varphi_{2}$ is $\eta$ on $\left[\mu_{1}, \mu_{2}\right]$ and 0 outside an $\varepsilon$-tubular neighborhood of that segment. Since $\bar{\Sigma}$ above $\left[\mu_{1}, \mu_{2}\right]$ is compact (see the proof of Theorem 4.2) one may choose $\varepsilon$ small enough such that the points on $\left[\mu_{1}, \mu_{2}\right]$ at a distance less than $\varepsilon$ from the endpoints are not covered by $\Sigma$. Let $\Sigma_{\mu} \equiv \Sigma \cap\left(\left[\mu_{1}, \mu_{2}\right] \times E\right)$ and $U_{\mu} \equiv U \cap\left(\left[\mu_{1}, \mu_{2}\right] \times E\right)$. Then, from Properties 2.2, 2.9, 2.6 and 2.10, $\|x\|-\varphi_{2}(\lambda)$ is 0-epi on $\Sigma_{\mu} \cap\left(U_{\mu}-\Sigma_{0}\right)$, hence one is reduced to a one-parameter problem. Since $\bar{\Sigma}_{\mu}$ is compact and away from $\partial U_{\mu}$, one may increase $\eta$ above the maximum of $\|x\|$ on $\bar{\Sigma}_{\mu}$, thus contradicting Property 2.1. Finally if for any pair $\mu_{1}, \mu_{2}, \bar{\Sigma}_{\mu}$ is bounded and contained in $U$, then, if $\mu_{1}$ is not covered by $\Sigma, \Sigma$ must cover $\mu_{2}$ and any other point in the component of $\lambda_{2}$. Q.E.D.

Remark 4.13. If $\Sigma$ is given by the zeros of $x-T(\lambda) x-h(\lambda, x)$, as in Proposition 4.5, and if $T_{0}$ is the set of $\lambda$ 's such that $I-T(\lambda)$ is not invertible, then clearly the local index of $I-T(\lambda)$ is constant on each connected component of $U_{0}-T_{0}$ and so $T_{0}$ disconnects $U_{0}$. Note that in this case, the points of $T_{0}$ are given locally (Lemma 4.2) by the singular points of some matrix $B(\lambda)$. However, by similarity, $\operatorname{det} B(\lambda)$ is defined globally and $T_{0}$ is given by the zeros of $\operatorname{det} B(\lambda)$. One may then apply the results for continuation, in particular Proposition 4.4 on the map $(\tilde{\lambda}$, $\operatorname{det} B(\lambda))$ which is 0 -epi on $U_{0}$ if and only if $\operatorname{det} B(\lambda)$ has different signs at $\lambda_{1}$ and $\lambda_{2}$.

Remark 4.14. Proposition 4.8 is a primitive version of duality between essential maps on $\Sigma_{0}$ and connectedness of $U_{0}-\Sigma_{0}$. In case $\tilde{\Lambda}>1$, it would be interesting to go deeper into this duality.

EXAMPLE 4.1. Let

$$
\begin{aligned}
f\left(x, \lambda_{1}, \lambda_{2}\right)= & x\left(\lambda_{1}^{2}+\lambda_{2}^{2}-2 \lambda_{2}\right)\left(\lambda_{1}^{2}+\left(\lambda_{2}-1\right)^{2}\right) \\
& +x^{3}\left(2 \lambda_{1}^{2}+2\left(\lambda_{2}-1\right)^{2}+1\right)+x^{5} \\
= & x\left[\left(\lambda_{1}^{2}+\left(\lambda_{2}-1\right)^{2}+x^{2}\right)^{2}-\left(\lambda_{1}^{2}+\left(\lambda_{2}-1\right)^{2}-x^{2}\right)\right] .
\end{aligned}
$$

The zeros of $f\left(x, \lambda_{1}, \lambda_{2}\right)$ correspond either to $x=0$, or to a lemniscate centered at $(0,0,1)$ and rotated around the $x$-axis. From Proposition 4.6, $\left(f\left(x, \lambda_{1}, \lambda_{2}\right), \lambda_{1}, x^{2}-\right.$ $\eta^{2}$ ) has degree 2, so that from Proposition 4.8, $\Sigma_{0}$ is the union of the circle $\lambda_{1}^{2}+\left(\lambda_{2}-1\right)^{2}=1$ and of the point $(0,1)$. Since $\Sigma$ is connected, $\Sigma$ is either the upper or the lower part of the lemniscate. Note that at the point $(0,1)$ the generalized index of $\left(f\left(x, \lambda_{1}, \lambda_{2}\right), x^{2}-\eta^{2}\right)$, as an element of $\Pi_{2}\left(S^{1}\right)$, is zero. Now, it is known that if all bifurcation points on a bounded continuum of $\bar{S}$ are of the same type, then the algebraic sum of the local indices is 0 in the corresponding stable group (see [I,I, Chapters 1 and 2]). It would be interesting to know if a classification of bifurcation points is possible, for example if a point with nonzero index in $\Pi_{m}$ (the stable group for $\Pi_{m+k}\left(S^{k}\right)$ ) may be connected to a surface of points with nonzero index in $\Pi_{n}$ for $n<m$.

\section{REFERENCES}

[AA,I] J. C. Alexander and S. S. Antman, Global and local behavior of bifurcating multidimensional continua of solutions for multiparameter nonlinear eigenvalue problems, Arch. Rational Mech. Anal. 76 (1981), 339-355.

[AA,II] Global behavior of solutions of nonlinear equations depending on infinite-dimensional parameter, Indiana Univ. Math. J. 32 (1983), 39-62. 
[AF] J. C. Alexander and P. M. Fitzpatrick, The homotopy of certain spaces of nonlinear operators and its relation to global bifurcation of the fixed points of parametrized condensing operators, J. Funct. Anal. 34 (1979), 87-106.

[AMP] J. C. Alexander, I. Massabó and J. Pejsachowicz, On the connectivity properties of the solution set of infinitely-parametrized families of vector fields, Boll. Un. Mat. Ital. A (6) 1 (1982), 309-312.

[Bo] Yu. G. Borisovich, Topology and non-linear functional analysis, Russian Math. Surveys 34 (1979), $14-23$.

[Br] F. E. Browder, Degree for nonlinear mappings of monotone type, Proc. Nat. Acad. Sci. U.S.A. 50 (1983), 1771-1773.

[BP] F. E. Browder and W. V. Petryshyn, Approximation methods and the generalized topological degree for nonlinear mappings in Banach spaces, J. Funct. Anal. 3 (1969), 385-414.

[BZS] Yu. G. Borisovich, V. G. Zvyagin and Yu. I. Sapronov, Nonlinear Fredholm maps and the Leray-Schauder theory, Russian Math. Surveys 32 (1977), 1-54.

[D] J. Dugundji, Topology, Allyn \& Bacon, Boston, Mass., 1966.

[FMP,I] P. M. Fitzpatrick, I. Massabó and J. Pejsachowicz, On the covering dimension of the set of solutions of some nonlinear equations, Trans. Amer. Math. Soc. (to appear).

[FMP,II] _ Global several-parameter bifurcation and continuation theorems: a unified approach via complementing maps, Math. Ann. 263 (1983), 61-73.

[FMV,I] M. Furi, M. Martelli and A. Vignoli, Contributions to the spectral theory for nonlinear operators in Banach spaces, Ann. Mat. Pura Appl. 118 (1978), 229-294.

[FMV,II] _ On the solvability of nonlinear operator equations in normed spaces, Ann. Mat. Pura Appl. 124 (1980), 321-343.

[FP] M. Furi and M. P. Pera, On the existence of an unbounded connected component of solutions for non-linear equations in Banach spaces, Rend. Accad. Naz. Lincei 57 (1979), 31-38.

[G] A. Granas, The theory of compact vector fields and some applications to the topology of functional spaces, Rozprawy Mat. 30 (1962).

[GG] K. Geba and A. Granas, Infinite dimensional cohomology theories, J. Math. Pures Appl. 52 (1973), 145-170.

[GT] J. Grispolakis and E. D. Tymchatyn, On confluent mappings and essential mappings - a survey, Rocky Mount. J. Math. 11 (1981), 131-153.

[I,I] J. Ize, Bifurcation theory for Fredholm operators, Mem. Amer. Math. Soc. No. 174 (1976).

[I,II] __ Introduction to bifurcation theory, Differential Equations, Lecture Notes in Math., vol. 957, Springer-Verlag, 1982, pp. 145-203.

[I,III] _ Obstruction theory and multiparameter Hopf bifurcation, Trans. Amer. Math. Soc. 289 (1985), 757-792.

[K] K. Kuratowski, Topology, Vols. I and II, Academic Press, New York, 1968.

[LS] J. Leray and J. Schauder, Topologie et équations fonctionelles, Ann. Sci. École Norm. Sup. 51 (1934), 45-78.

[M] J. Mawhin, Equivalence theorems for nonlinear operator equations and coincidence degree theory for some mappings in locally convex topological vector space, J. Differential Equations 12 (1972), 610-636.

[MP] I. Massabó and J. Pejsachowicz, On the connectivity properties of the solution set of parametrized families of compact vector fields, J. Funct. Anal. 59 (1984), 151-166.

[N] R. D. Nussbaum, The fixed point index for local condensing maps, Ann. Mat. Pura Appl. 89 (1971), 217-258.

[P] A. R. Pears, Dimension theory of general spaces, Cambridge Univ. Press, 1975.

[R] P. H. Rabinowitz, Some global results for nonlinear eigenvalue problems, J. Funct. Anal. 7 (1971), 487-513.

[S] B. N. Sadovskij, Limit compact and condensing operators, Russian Math. Surveys 27 (1972), 85-155.

[Sp] E. H. Spanier, Algebraic topology, McGraw-Hill, New York, 1966.

[W] G. T. Whyburn, Topological analysis, Princeton Univ. Press, Princeton, N. J., 1958.

iImaS-Unam, Apdo. Postal 20-726, Admón No. 20, Deleg. Alvaro Obregon, 01000 Mexico, D. F., Mexico (Current address of J. Ize)

Dipartimento di Matematica, Università della Calabria, 87030 arcavacata di Rende (CS), CAlabria, Italy (Current address of I. Massabó and J. Pejsachowicz)

Dipartimento di Matematica, II Universita di Roma, Via Orazio Raimondo, 00173 Roma, Italy (Current address of A. Vignoli) 